\title{
The Aryne [2,3] Stevens Rearrangement
}

Tony Roy, ${ }^{\dagger, \S}$ Manikandan Thangaraj, ${ }^{\dagger, \$}$ Trinadh Kaicharla, ${ }^{\dagger, \&}{ }^{2}$ Rupa V. Kamath, ${ }^{\dagger}$ Rajesh G. Gonnade, ${ }^{\ddagger}$ and Akkattu T. Biju* ${ }^{*}, \$$

${ }^{\dagger}$ Organic Chemistry Division, ${ }^{\star}$ Centre for Materials Characterization, CSIRNational Chemical Laboratory (CSIR-NCL), Dr. Homi Bhabha Road, Pune 411008, India

${ }^{\S}$ Academy of Scientific and Innovative Research (AcSIR), New Delhi 110020, India at.biju@ncl.res.in

\section{Supporting Information}

1. General Information $\quad$ S2

2. General Procedure for the preparation of Allyl Aniline Substrates

3. General Procedure for the Aryne [2,3] Stevens Rearrangement S3

4. Procedure for the Enantiospecific [2,3] Stevens Rearrangement

5. X- ray Data of $\mathbf{3 r} \quad$ S4

6. Synthesis and Characterization of Homoallylic Amines S6

7. ${ }^{1} \mathrm{H}$ and ${ }^{13} \mathrm{C}$ NMR Spectra of Homoallylic Amines $\quad$ S25

8. HPLC data of Chiral Proline Derivatives $\quad$ S57 


\section{General Information}

Unless otherwise specified, all reactions were carried out under an atmosphere of argon in flame-dried reaction vessels with Teflon screw caps. $30{ }^{\circ} \mathrm{C}$ corresponds to the room temperature of the lab when the experiments were carried out. THF was freshly purified by distillation over Na-benzophenone and was transferred under argon. 18-Crown-6 was recrystallized from dry $\mathrm{CH}_{3} \mathrm{CN}$ and $\mathrm{KF}$ was dried by heating at $110^{\circ} \mathrm{C}$ for $12 \mathrm{~h}$ and left to cool under argon and stored in argon filled glove-box. The 2(trimethylsilyl)phenyl trifluoromethanesulfonate 1a and the other symmetric and unsymmetric aryne precursors were synthesized following literature procedure. ${ }^{1}$

Analytical thin layer chromatography was performed on TLC Silica gel 60 F254. Visualization was accomplished with short wave UV light. Chromatography was performed on silica gel (230400 mesh) by standard techniques eluting with solvents as indicated.

All compounds were fully characterized. ${ }^{1} \mathrm{H}$ and ${ }^{13} \mathrm{C}$ NMR spectra were recorded on Bruker AV 400, 500 in solvents as indicated. Chemical shifts $(\delta)$ are given in ppm. The residual solvent signals were used as references and the chemical shifts converted to the TMS scale $\left(\mathrm{CDCl}_{3}\right.$ : $\delta \mathrm{H}=$ $7.26 \mathrm{ppm}, \delta \mathrm{C}=77.16 \mathrm{ppm})$. Infrared spectra were recorded on a Bruker Alpha-E Infrared Spectrophotometer. The wave numbers (n) of recorded IR-signals are quoted in $\mathrm{cm}^{-1}$. HRMS data were recorded on either Thermo Scientific Q-Exactive, Accela 1250 pump. HPLC analysis was performed on Agilent Technologies 1260 Infinity with UV detector. Optical rotation was measured with a JASCO P 2000 digital polarimeter at rt using $50 \mathrm{~mm}$ cell of $1 \mathrm{~mL}$ capacity.

\footnotetext{
${ }^{1}$ (a) Sato, Y.; Tamura, T.; Kinbara, A.; Morib, M. Adv. Synth. Catal. 2007, 349, 647. (b) Peña, D.; Cobas,
} A.; Pérez, D.; Guitián, E. Synthesis 2002, 1454. 


\section{General Procedure for the preparation of Allyl Aniline Substrates}

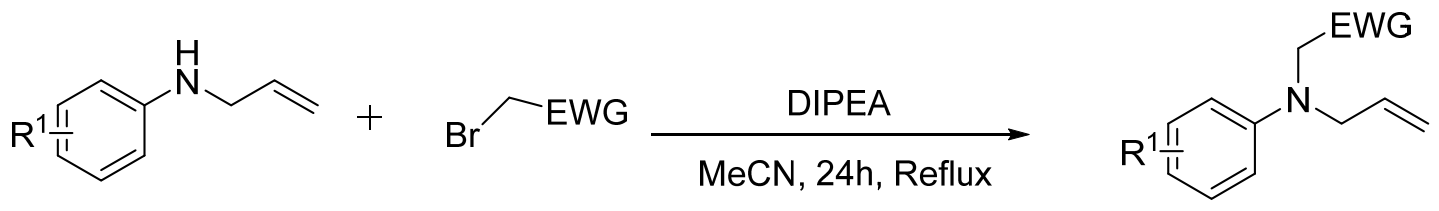

Following the literature procedure, the allyl aniline is treated with the corresponding bromo compounds to afford the allyl amine starting materials for the aryne [2,3] Stevens rearrangement. ${ }^{2}$

\section{General Procedure for the Aryne [2,3] Stevens Rearrangement}
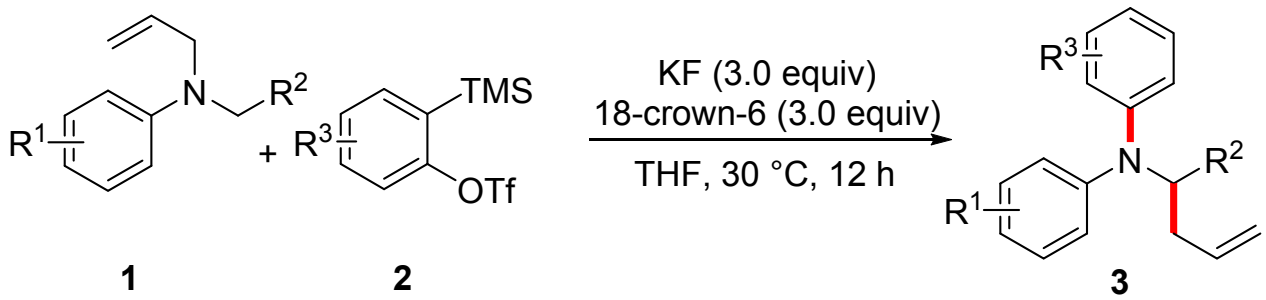

To a flame-dried screw-capped test tube equipped with a magnetic stir bar was added the $\mathrm{KF}(87 \mathrm{mg}, 1.5 \mathrm{mmol})$ and 18-crown-6 $(0.396 \mathrm{mg}, 1.5 \mathrm{mmol})$ in a glove box. The mixture was dissolved in THF (2.0 mL) under argon atmosphere. Allyl amine $1(0.50 \mathrm{mmol})$ was added outside the glove box under argon atmosphere. To the stirring solution, aryne precursor $2(0.75$ mmol) was added. Then the reaction mixture was allowed to react at $30{ }^{\circ} \mathrm{C}$ for $12 \mathrm{~h}$. After $12 \mathrm{~h}$, the reaction was stopped, the solvent was evaporated and the crude residue pre-adsorbed on silica gel and purified by flash column chromatography (Pet. ether $/$ EtOAc $=99 / 01)$ on silica gel to afford the corresponding homoallylic amines $\mathbf{3}$ in moderate to good yields.

\footnotetext{
${ }^{2}$ Everett, R. K.; John P. Wolfe, J, P. J. Org. Chem., 2015, 80, 9041.
} 


\section{General Procedure for the Enantiospecific [2,3] Stevens Rearrangement}

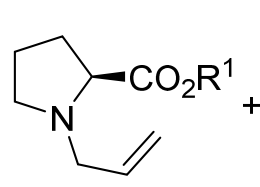

6

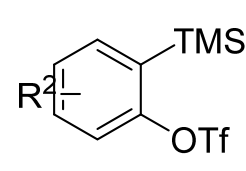

2

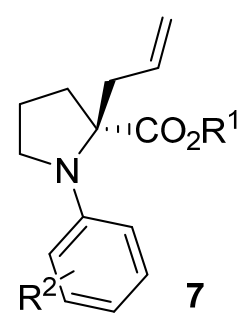

To a flame-dried screw-capped test tube equipped with a magnetic stir bar was added the $\mathrm{KF}(87 \mathrm{mg}, 1.5 \mathrm{mmol})$ and 18-crown-6 $(0.396 \mathrm{mg}, 1.5 \mathrm{mmol})$ in a glove box. The mixture was dissolved in THF $(2.0 \mathrm{~mL})$ under argon atmosphere. The $N$-allyl proline derivative $6(0.50$ mmol) was added outside the glove box under argon atmosphere. To the stirring solution aryne precursor $2(0.75 \mathrm{mmol})$ was added. Then the reaction mixture was allowed to react at $30{ }^{\circ} \mathrm{C}$ for $2 \mathrm{~h}$. After $2 \mathrm{~h}$ the reaction was stopped, the solvent was evaporated and the crude residue preadsorbed on silica gel and purified by flash column chromatography (Pet. ether $/$ EtOAc $=99 / 01)$ on silica gel to afford the corresponding $N$-aryl proline derivatives 7 in moderate to good yields.

\section{X-ray Data of 3r}

X-ray intensity data measurements of compound $\mathbf{3 r}$ was carried out on a Bruker D8 VENTURE Kappa Duo PHOTON II CPAD diffractometer equipped with Incoatech multilayer mirrors optics. The intensity measurements were carried out with micro-focus sealed tube diffraction source $\left(\mathrm{MoK}_{\alpha}=0.71073 \AA\right)$ at $150(2) \mathrm{K}$ temperature using OXFORD LN2 cryosystem. The X-ray generator was operated at $50 \mathrm{kV}$ and $1.4 \mathrm{~mA}$. A preliminary set of cell constants and an orientation matrix were calculated from three sets of 36 frames. Data were collected with $\omega$ scan width of $0.5^{\circ}$ at different settings of $\varphi$ and $2 \theta$ with a frame time of 15 secs keeping the sample-to-detector distance fixed at $4.00 \mathrm{~cm}$. The X-ray data 
collection was monitored by APEX3 program (Bruker, 2016). ${ }^{3}$ All the data were corrected for Lorentzian, polarization and absorption effects using SAINT and SADABS programs (Bruker, 2016). SHELX-97 was used for structure solution and full matrix least-squares refinement on $F^{2}{ }^{2}$ All the hydrogen atoms were placed in a geometrically idealized position and constrained to ride on their parent atoms.. The molecules of compound $\mathbf{3 r}$ crystallized in tricinic $P-1$ space group containing two symmetry independednt moleucles in the asymmetriuc unit. The allyl moiety of one of the molecules showed statistical disorder over two position with occupancy $40 \%$ and $60 \%$. An ORTEP III $^{3}$ view of unprimed moleucle was drawn with $50 \%$ probability displacement ellipsoids and $\mathrm{H}$ atoms are shown as small spheres of arbitrary radii.

Crystal data of 3r: $\left(\mathrm{C}_{23} \mathrm{H}_{21} \mathrm{NO}\right), \mathrm{M}=327.41$, colorless block crystals, $0.27 \times 0.24 \times$ $0.22 \mathrm{~mm}^{3}$, triclinic, space group $P-1, a=10.2062(6) \AA, b=13.2507(8) \AA, c=13.9258(8) \AA$, $\alpha=91.429(2)^{\circ}, \beta=104.805(2)^{\circ}, \gamma=98.033(2)^{\circ}, V=1799.30(18) \AA^{3}, Z=4, T=150(2) \mathrm{K}$, $2 \theta_{\max }=61.096^{\circ}, D_{\text {calc }}\left(\mathrm{g} \mathrm{cm}^{-3}\right)=1.209, F(000)=696, \mu\left(\mathrm{mm}^{-1}\right)=0.073,126850$ reflections collected, 10988 unique reflections $\left(R_{\mathrm{int}}=0.0659\right), 8900$ observed $(I>2 \sigma(I))$ reflections, multi-scan absorption correction, $T_{\min }=0.980, T_{\max }=0.984,469$ refined parameters, $S=$ $1.041, R 1=0.0536, w R 2=0.1215$ (all data $R=0.0684, w R 2=0.1288$ ), maximum and minimum residual electron densities; $\Delta \rho_{\max }=0.32, \Delta \rho_{\min }=-0.20\left(\mathrm{e} \AA^{-3}\right)$.

3 (a) Bruker (2016). APEX3, SAINT and SADABS. Bruker AXS Inc., Madison, Wisconsin, USA. (b) Sheldrick, G. M. Acta Crystallogr. 2008, A64, 112. (c) Farrugia, L. J. J. Appl. Cryst. 1997, 30, 565. 


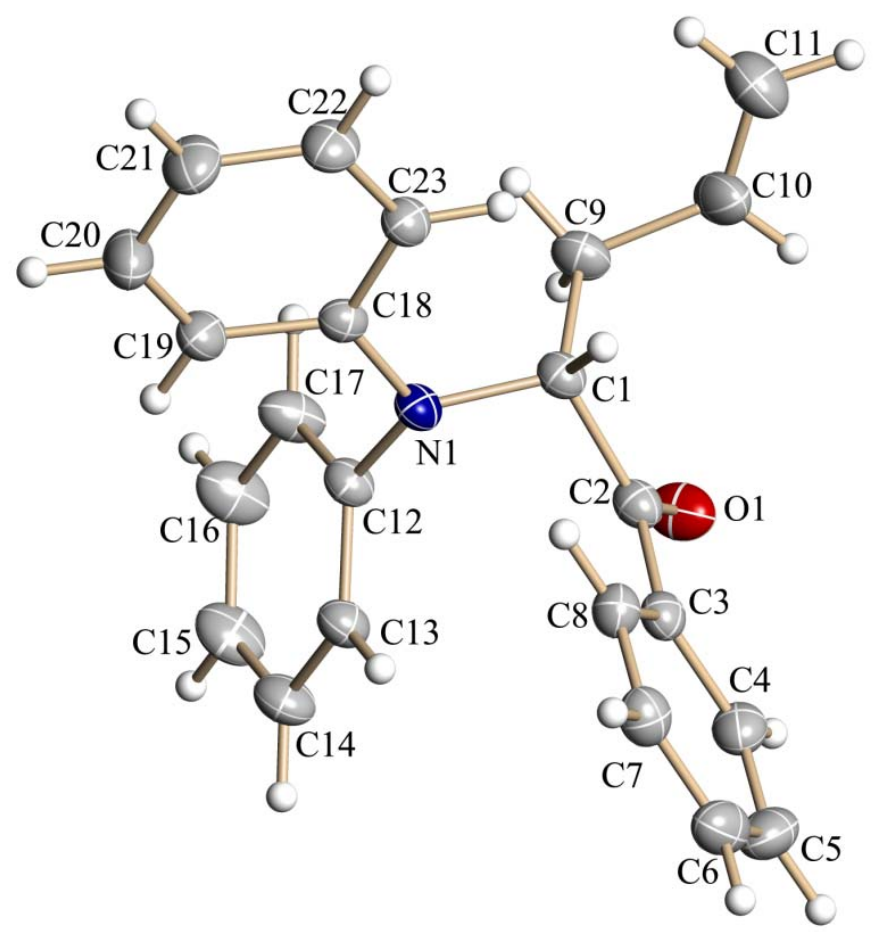

Ortep Diagram of 3r (thermal ellipsoids are shown with 50\% probability).

\section{Synthesis and Characterization of Homoallylic Amines}

\section{Ethyl-2-(diphenylamino)pent-4-enoate (3a)}

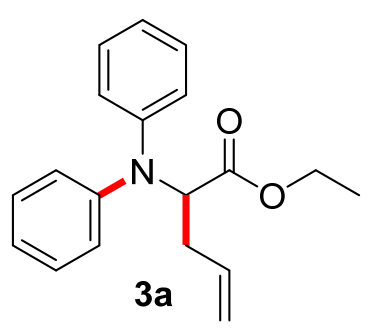

Following the general procedure, treatment of ethyl $\mathrm{N}$-allyl- $N$ phenylglycinate 1a $(0.110 \mathrm{~g}, 0.5 \mathrm{mmol})$ and 2-(trimethylsilyl)phenyl trifluoromethane sulfonate $2 \mathbf{a}(0.223 \mathrm{~g}, 182 \mu \mathrm{L}, 0.75 \mathrm{mmol})$ in the presence of $\mathrm{KF}(0.087 \mathrm{~g}, 1.5 \mathrm{mmol})$ and 18-crown-6 (0.396 g, 1.5 mmol) in THF $(2.0 \mathrm{~mL})$ at $\mathrm{rt}$ for $12 \mathrm{~h}$ followed by flash column chromatography $($ Pet. ether $/$ EtOAc $=99 / 01)$ of the crude reaction mixture using silica gel afforded ethyl-2-(diphenylamino)pent-4-enoate 3a as yellow oil (0.128 g, 87\% yield).

$\boldsymbol{R}_{\mathbf{f}}($ Pet. ether $/$ EtOAc $=95 / 05): 0.53 ;{ }^{1} \mathbf{H}$ NMR (400 MHz, $\left.\mathbf{C D C l}_{3}\right) \delta 7.31(\mathrm{t}, J=7.8 \mathrm{~Hz}, 4 \mathrm{H})$, $7.06-7.03(\mathrm{~m}, 6 \mathrm{H}), 5.91-5.81(\mathrm{~m}, 1 \mathrm{H}), 5.12-5.07(\mathrm{~m}, 2 \mathrm{H}), 4.69(\mathrm{t}, J=7.5 \mathrm{~Hz}, 1 \mathrm{H}), 4.29-4.17$ $(\mathrm{m}, 2 \mathrm{H}), 2.69$ (t, $J=7.0 \mathrm{~Hz}, 2 \mathrm{H}), 1.27$ (t, $J=7.1 \mathrm{~Hz}, 3 \mathrm{H}) .{ }^{13} \mathbf{C}$ NMR (100 MHz, CDCl $\left.\mathbf{3}\right) \delta$ $172.77,146.67,134.56,129.19,122.68,122.30,117.71,62.72,61.12,34.48,14.27 . \mathbf{H M S}$ (ESI) calculated $[\mathrm{M}+\mathrm{H}]^{+}$for $\mathrm{C}_{19} \mathrm{H}_{22} \mathrm{O}_{2} \mathrm{~N}: 296.1645$, found: 296.1637. FTIR (cm $\left.{ }^{-1}\right)$ 3022, 2951, 1722, 1598, 1517, 1445, 1363, 1289, 1261, 1218, 1082, 951, 909, 757. 


\section{Ethyl-2-((4-methoxyphenyl)(phenyl)amino)pent-4-enoate (3b)}

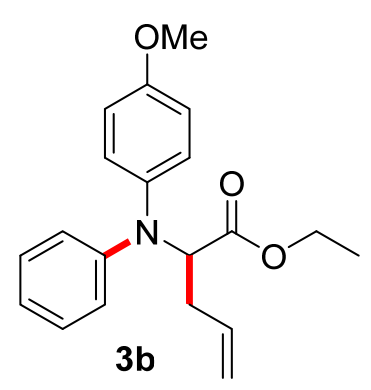

Following the general procedure, treatment of ethyl N-allyl-N-(4methoxyphenyl)glycinate $\mathbf{1 b}(0.125 \mathrm{~g}, \quad 0.5 \mathrm{mmol})$ and 2(trimethylsilyl)phenyl trifluoromethane sulfonate $\mathbf{2 a}(0.223 \mathrm{~g}, 182 \mu \mathrm{L}$, $0.75 \mathrm{mmol})$ in the presence of $\mathrm{KF}(0.087 \mathrm{~g}, 1.5 \mathrm{mmol})$ and 18-crown-6 $(0.396 \mathrm{~g}, 1.5 \mathrm{mmol})$ in THF $(2.0 \mathrm{~mL})$ at $30{ }^{\circ} \mathrm{C}$ for $12 \mathrm{~h}$ followed by flash column chromatography $($ Pet. ether $/$ EtOAc $=99 / 01)$ of the crude reaction mixture using silica gel afforded ethyl-2-((4-methoxyphenyl)(phenyl)amino)pent-4enoate $3 \mathbf{b}$ as yellow oil ( $0.144 \mathrm{~g}, 88 \%$ yield).

$\boldsymbol{R}_{\mathbf{f}}\left(\right.$ Pet. ether $/$ EtOAc = 95/05): 0.48; ${ }^{1} \mathbf{H}$ NMR (400 MHz, $\left.\mathbf{C D C l}_{3}\right) \delta 7.27$ - $7.09(\mathrm{~m}, 4 \mathrm{H}), 6.93$ $(\mathrm{d}, J=8.8 \mathrm{~Hz}, 2 \mathrm{H}), 6.79(\mathrm{t}, J=7.2 \mathrm{~Hz}, 1 \mathrm{H}), 6.68(\mathrm{~d}, J=8.2 \mathrm{~Hz}, 2 \mathrm{H}), 5.87-5.77(\mathrm{~m}, 1 \mathrm{H}), 5.09$ (s, 1H), $5.06(\mathrm{~d}, J=5.9 \mathrm{~Hz}, 1 \mathrm{H}), 4.62(\mathrm{t}, J=7.5 \mathrm{~Hz}, 1 \mathrm{H}), 4.24-4.18(\mathrm{~m}, 2 \mathrm{H}), 3.85(\mathrm{~s}, 3 \mathrm{H}), 2.59$ $(\mathrm{t}, J=7.1 \mathrm{~Hz}, 2 \mathrm{H}), 1.27(\mathrm{t}, J=7.1 \mathrm{~Hz}, 3 \mathrm{H}) .{ }^{13} \mathbf{C}$ NMR (100 MHz, CDCl $\left.\mathbf{3}\right) \delta 172.96,157.65$, $148.77,137.35,134.60,130.46,129.00,118.65,117.69,115.92,114.67,62.33,61.11,55.54$, 34.85, 14.35. HRMS (ESI) calculated $[\mathrm{M}+\mathrm{H}]^{+}$for $\mathrm{C}_{20} \mathrm{H}_{24} \mathrm{O}_{3} \mathrm{~N}: 326.1751$, found: 326.1747 . FTIR (cm $\left.{ }^{-1}\right)$ 3018, 2983, 2909, 2402, 1732, 1597, 1509, 1245, 1183, 1035, 993, 923, 772, 668, 501.

\section{Ethyl -2-(phenyl(p-tolyl)amino)pent-4-enoate (3c)}

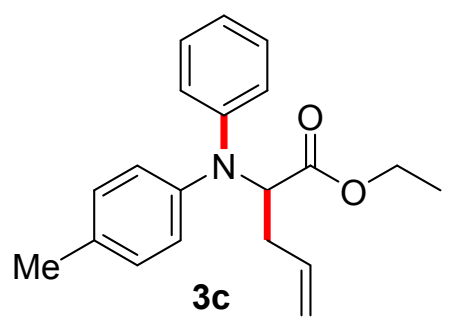

Following the general procedure, treatment of ethyl $N$-allyl-N- $(p$ tolyl)glycinate 1c (0.117 g, $0.5 \mathrm{mmol})$ and 2-(trimethylsilyl)phenyl trifluoromethane sulfonate $2 \mathbf{a}(0.223 \mathrm{~g}, 182 \mu \mathrm{L}, 0.75 \mathrm{mmol})$ in the presence of KF (0.087 g, $1.5 \mathrm{mmol})$ and 18-crown-6 (0.396 g, 1.5 $\mathrm{mmol})$ in THF $(2.0 \mathrm{~mL})$ at $30^{\circ} \mathrm{C}$ for $12 \mathrm{~h}$ followed by flash column chromatography $($ Pet. ether $/$ EtOAc $=99 / 01)$ of the crude reaction mixture using silica gel afforded ethyl -2-(phenyl( $p$-tolyl)amino)pent-4-enoate 3c as yellow oil $(0.133 \mathrm{~g}, 86 \%$ yield).

$\boldsymbol{R}_{\mathbf{f}}\left(\right.$ Pet. ether $/$ EtOAc $=$ 95/05): 0.52; ${ }^{1} \mathbf{H}$ NMR (400 $\left.\mathbf{M H z}, \mathbf{C D C l}_{3}\right) \delta 7.25(\mathrm{t}, J=7.9 \mathrm{~Hz}, 2 \mathrm{H})$, $7.17(\mathrm{~d}, J=8.2 \mathrm{~Hz}, 2 \mathrm{H}), 7.06(\mathrm{~d}, J=8.3 \mathrm{~Hz}, 2 \mathrm{H}), 6.94-6.87(\mathrm{~m}, 3 \mathrm{H}), 5.91-5.80(\mathrm{~m}, 1 \mathrm{H}), 5.12$ $5.08(\mathrm{~m}, 2 \mathrm{H}), 4.65(\mathrm{t}, J=7.5 \mathrm{~Hz}, 1 \mathrm{H}), 4.26-4.19(\mathrm{~m}, 2 \mathrm{H}), 2.66(\mathrm{t}, J=7.1 \mathrm{~Hz}, 2 \mathrm{H}), 2.38(\mathrm{~s}, 3 \mathrm{H})$, $1.28(\mathrm{t}, J=7.1 \mathrm{~Hz}, 3 \mathrm{H}) .{ }^{13} \mathbf{C}$ NMR (100 MHz, $\left.\mathbf{C D C l}_{3}\right) \delta 172.89,147.74,143.22,134.66,133.59$, 129.97, 129.06, 125.74, 120.46, 119.49, 117.67, 62.64, 61.10, 34.59, 20.94, 14.31. HRMS (ESI) 
calculated $[\mathrm{M}+\mathrm{H}]^{+}$for $\mathrm{C}_{20} \mathrm{H}_{24} \mathrm{O}_{2} \mathrm{~N}$ : 310.1802 , found: 310.1800 . FTIR $\left(\mathbf{c m}^{-1}\right)$ 3026, 2936, 1718 , $1588,1523,1453,1369,1311,1227,1108,998$.

\section{Ethyl-2-((4-bromophenyl)(phenyl)amino)pent-4-enoate (3d)}

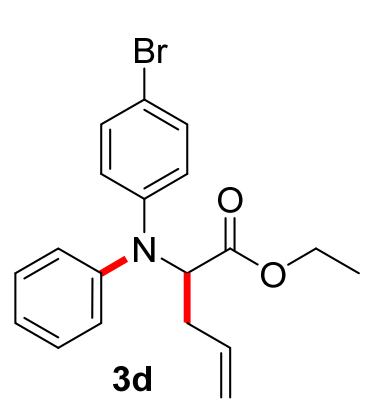

Following the general procedure, treatment of ethyl $\mathrm{N}$-allyl- $\mathrm{N}-(4-$ bromophenyl)glycinate $\quad \mathbf{1 d} \quad(0.149 \quad \mathrm{~g}, \quad 0.5 \quad \mathrm{mmol})$ and $2-$ (trimethylsilyl)phenyl trifluoromethane sulfonate $2 \mathrm{a}(0.223 \mathrm{~g}, 182 \mu \mathrm{L}$, $0.75 \mathrm{mmol})$ in the presence of $\mathrm{KF}(0.087 \mathrm{~g}, 1.5 \mathrm{mmol})$ and 18 -crown-6 $(0.396 \mathrm{~g}, 1.5 \mathrm{mmol})$ in THF $(2.0 \mathrm{~mL})$ at $30{ }^{\circ} \mathrm{C}$ for $12 \mathrm{~h}$ followed by flash column chromatography (Pet. ether $/ \mathrm{EtOAc}=99 / 01)$ of the crude reaction mixture using silica gel afforded ethyl-2-((4-bromophenyl)(phenyl)amino)pent-4-enoate 3d as yellow oil ( $0.156 \mathrm{~g}, 83 \%$ yield).

$\boldsymbol{R}_{\mathbf{f}}($ Pet. ether $/$ EtOAc $=95 / 05): 0.57 ;{ }^{1} \mathbf{H}$ NMR $\left(400 \mathbf{~ M H z}, \mathbf{C D C l}_{3}\right) \delta 7.38-7.27(\mathrm{~m}, 4 \mathrm{H}), 7.13-$ $7.06(\mathrm{~m}, 3 \mathrm{H}), 6.80(\mathrm{~d}, J=8.8 \mathrm{~Hz}, 2 \mathrm{H}), 5.99-5.66(\mathrm{~m}, 1 \mathrm{H}), 5.10-5.04(\mathrm{~m}, 2 \mathrm{H}), 4.61(\mathrm{t}, J=7.5$ $\mathrm{Hz}, 1 \mathrm{H}), 4.30$ - $4.14(\mathrm{~m}, 2 \mathrm{H}), 2.74-2.44(\mathrm{~m}, 2 \mathrm{H}), 1.26$ (t, $J=7.1 \mathrm{~Hz}, 3 \mathrm{H}) .{ }^{13} \mathbf{C}$ NMR (100 MHz, $\left.\mathbf{C D C l}_{3}\right) \delta 172.56,146.27,145.90,134.32,132.06,129.58,129.47,124.48,123.73,122.63$, 118.01, 114.59, 113.98, 62.80,61.34, 34.37, 14.34. HRMS (ESI) calculated $[\mathrm{M}+\mathrm{H}]^{+}$for $\mathrm{C}_{19} \mathrm{H}_{21} \mathrm{O}_{2} \mathrm{NBr}$ : 374.0750, found: 374.0749. FTIR (cm $\left.{ }^{-1}\right)$ 3066, 3017, 2938, 1732, 1585, 1444, 1377, 1217, 1097, 924, 771, 668 .

\section{Ethyl-2-((4-chlorophenyl)(phenyl)amino)pent-4-enoate (3e)}

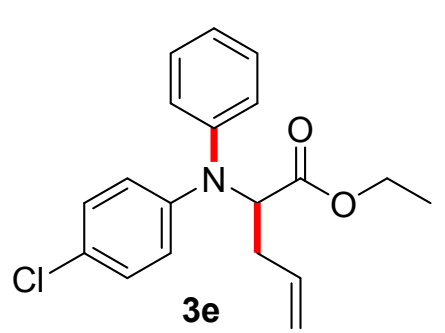

Following the general procedure, treatment of ethyl $\mathrm{N}$-allyl-N-(4chlorophenyl)glycinate $\quad 1 \mathrm{e} \quad(0.127 \mathrm{~g}, \quad 0.5 \mathrm{mmol})$ and $\quad 2$ (trimethylsilyl)phenyl trifluoromethane sulfonate $2 \mathrm{a}(0.223 \mathrm{~g}, 182$ $\mu \mathrm{L}, 0.75 \mathrm{mmol})$ in the presence of $\mathrm{KF}(0.087 \mathrm{~g}, 1.5 \mathrm{mmol})$ and 18 crown-6 (0.396 g, $1.5 \mathrm{mmol})$ in THF $(2.0 \mathrm{~mL})$ at $30^{\circ} \mathrm{C}$ for $12 \mathrm{~h}$ followed by flash column chromatography (Pet. ether $/ \mathrm{EtOAc}=99 / 01)$ of the crude reaction mixture using silica gel afforded ethyl-2-((4-chlorophenyl)(phenyl)amino)pent-4-enoate $\mathbf{3 e}$ as yellow oil $(0.132 \mathrm{~g}, 80 \%$ yield $)$.

$\boldsymbol{R}_{\mathbf{f}}($ Pet. ether $/$ EtOAc $=95 / 05): 0.45 ;{ }^{1} \mathbf{H}$ NMR $\left(\mathbf{4 0 0} \mathbf{~ M H z}, \mathbf{C D C l}_{3}\right) \delta 7.31(\mathrm{t}, J=7.8 \mathrm{~Hz}, 2 \mathrm{H})$, $7.23(\mathrm{~d}, J=8.6 \mathrm{~Hz}, 2 \mathrm{H}), 7.10-7.03(\mathrm{~m}, 3 \mathrm{H}), 6.90(\mathrm{~d}, J=8.5 \mathrm{~Hz}, 2 \mathrm{H}), 5.86-5.76(\mathrm{~m}, 1 \mathrm{H}), 5.11$ - 
$5.04(\mathrm{~m}, 2 \mathrm{H}), 4.62(\mathrm{t}, J=7.8 \mathrm{~Hz}, 1 \mathrm{H}), 4.25-4.18(\mathrm{~m}, 2 \mathrm{H}), 2.69-2.60(\mathrm{~m}, 2 \mathrm{H}), 1.26(\mathrm{t}, J=6.9$ $\mathrm{Hz}, 3 \mathrm{H}) .{ }^{13} \mathbf{C}$ NMR (100 MHz, $\left.\mathbf{C D C l}_{3}\right) \delta$ 172.61, 146.18, 145.58, 134.33, 129.41, 129.17, 126.92, 123.71, 123.26, 122.99, 117.98, 62.81, 61.32, 34.40, 14.32. HRMS (ESI) calculated $[\mathrm{M}+\mathrm{H}]^{+}$for $\mathrm{C}_{19} \mathrm{H}_{21} \mathrm{O}_{2} \mathrm{NCl}$ : 330.1255, found: 330.1258. FTIR $\left(\mathbf{c m}^{-1}\right)$ 3022, 2358, 1718, 1712, $1590,1509,1460,1359,1316,1278,1227,998$.

\section{Ethyl-2-((4-fluorophenyl)(phenyl)amino)pent-4-enoate (3f)}

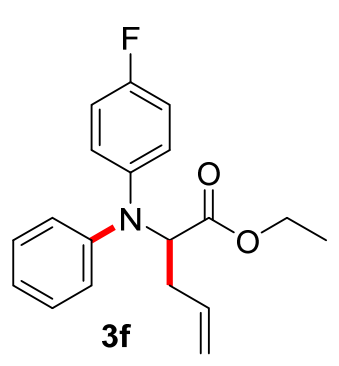

Following the general procedure, treatment of ethyl $\mathrm{N}$-allyl- $\mathrm{N}-(4-$ fluorophenyl)glycinate $\mathbf{1 f}(0.119 \mathrm{~g}, 0.5 \mathrm{mmol})$ and 2-(trimethylsilyl)phenyl trifluoromethane sulfonate $2 \mathrm{a}(0.223 \mathrm{~g}, 182 \mu \mathrm{L}, 0.75 \mathrm{mmol})$ in the presence of $\mathrm{KF}(0.087 \mathrm{~g}, 1.5 \mathrm{mmol})$ and 18-crown-6 (0.396 g, $1.5 \mathrm{mmol})$ in THF $(2.0 \mathrm{~mL})$ at $30{ }^{\circ} \mathrm{C}$ for $12 \mathrm{~h}$ followed by flash column chromatography $($ Pet. ether $/ \mathrm{EtOAc}=99 / 01)$ of the crude reaction mixture using silica gel afforded ethyl-2-((4-fluorophenyl)(phenyl)amino)pent-4-enoate $\mathbf{3 f}$ as yellow oil (0.141 g, 90\% yield).

$\boldsymbol{R}_{\mathbf{f}}($ Pet. ether $/$ EtOAc $=95 / 05): 0.50 ;{ }^{1} \mathbf{H}$ NMR $\left(400 \mathbf{~ M H z}, \mathbf{C D C l}_{3}\right) \delta 7.21(\mathrm{t}, J=7.8 \mathrm{~Hz}, 2 \mathrm{H})$, 7.16-7.12 (m, 2H), $7.02(\mathrm{t}, J=8.6 \mathrm{~Hz}, 2 \mathrm{H}), 6.89(\mathrm{t}, J=7.3 \mathrm{~Hz}, 1 \mathrm{H}), 6.78(\mathrm{~d}, J=8.1 \mathrm{~Hz}, 2 \mathrm{H})$, 5.84-5.74 (m, 1H), $5.19-4.91(\mathrm{~m}, 2 \mathrm{H}), 4.60(\mathrm{t}, J=7.5 \mathrm{~Hz}, 1 \mathrm{H}), 4.33-4.08(\mathrm{~m}, 2 \mathrm{H}), 2.74-2.47$ $(\mathrm{m}, 2 \mathrm{H}), 1.24(\mathrm{t}, J=7.1 \mathrm{~Hz}, 3 \mathrm{H}) .{ }^{13} \mathbf{C}$ NMR (100 MHz, $\left.\mathbf{C D C l}_{3}\right) \delta 172.80,159.92(\mathrm{~d}, J=244.5)$, $147.77,134.41,129.19,128.26$ (d, $J=8.44$ ), 120.47, 118.76, 117.89, 116.19, 115.97, 62.62, 61.24, 34.64, 14.34. HRMS (ESI) calculated $[\mathrm{M}+\mathrm{H}]{ }^{+}$for $\mathrm{C}_{19} \mathrm{H}_{21} \mathrm{O}_{2} \mathrm{NF}$ : 314.1551, found: 314.1546. FTIR $\left(\mathbf{c m}^{-1}\right)$ 3018, 2983, 2936, 1733, 1504, 1443, 1218, 1094, 994, 839, 772.

\section{Ethyl -2-(phenyl( $m$-tolyl)amino)pent-4-enoate (3g)}<smiles>C=CCC(C(=O)OCC)N(c1ccccc1)c1cccc(C)c1</smiles>

Following the general procedure, treatment of ethyl $N$-allyl- $N-(\mathrm{m}-$ tolyl)glycinate $1 \mathrm{~g}(0.117 \mathrm{~g}, 0.5 \mathrm{mmol})$ and 2-(trimethylsilyl)phenyl trifluoromethane sulfonate $2 \mathbf{a}(0.223 \mathrm{~g}, 182 \mu \mathrm{L}, 0.75 \mathrm{mmol})$ in the presence of KF (0.087 g, $1.5 \mathrm{mmol})$ and 18-crown-6 (0.396 g, 1.5 $\mathrm{mmol})$ in THF $(2.0 \mathrm{~mL})$ at $30^{\circ} \mathrm{C}$ for $12 \mathrm{~h}$ followed by flash column chromatography $($ Pet. ether $/$ EtOAc $=99 / 01)$ of the crude reaction mixture using silica gel afforded ethyl -2-(phenyl( $m$-tolyl)amino)pent-4-enoate $\mathbf{3 g}$ as yellow oil $(0.116 \mathrm{~g}, 75 \%$ yield $)$. 
$\boldsymbol{R}_{\mathbf{f}}($ Pet. ether $/$ EtOAc $=95 / 05): 0.51 ;{ }^{1} \mathbf{H}$ NMR (400 MHz, $\left.\mathbf{C D C l}_{3}\right) \delta 7.28(\mathrm{t}, J=7.8 \mathrm{~Hz}, 2 \mathrm{H})$, $7.18(\mathrm{t}, J=7.6 \mathrm{~Hz}, 1 \mathrm{H}), 7.03$ - $6.97(\mathrm{~m}, 3 \mathrm{H}), 6.88$ - $6.82(\mathrm{~m}, 3 \mathrm{H}), 5.87$ - $5.78(\mathrm{~m}, 1 \mathrm{H}), 5.10$ - 5.05 $(\mathrm{m}, 2 \mathrm{H}), 4.64(\mathrm{t}, J=7.5 \mathrm{~Hz}, 1 \mathrm{H}), 4.24-4.17(\mathrm{~m}, 2 \mathrm{H}), 2.65(\mathrm{t}, J=6.8 \mathrm{~Hz}, 2 \mathrm{H}), 2.31(\mathrm{~s}, 3 \mathrm{H}), 1.26$ $(\mathrm{t}, J=7.1 \mathrm{~Hz}, 3 \mathrm{H}) .{ }^{13} \mathbf{C}$ NMR (100 $\left.\mathbf{~ M H z , ~} \mathbf{C D C l}_{3}\right) \delta 172.90,146.83,146.59,139.05,134.70$, $129.18,129.06,123.83,123.47,122.31,122.01,120.23,117.72$, 62.76, 61.15, 34.60, 21.71, 14.34. HRMS (ESI) calculated $[\mathrm{M}+\mathrm{H}]^{+}$for $\mathrm{C}_{20} \mathrm{H}_{24} \mathrm{O}_{2} \mathrm{~N}: 310.1802$, found: 310.1799 . FTIR (cm ${ }^{-}$ 1) $3027,2939,1716,1592,1523,1441,1357,1315,1278,1118,995$.

\section{Ethyl 2-((3-chlorophenyl)(phenyl)amino)pent-4-enoate (3h)}

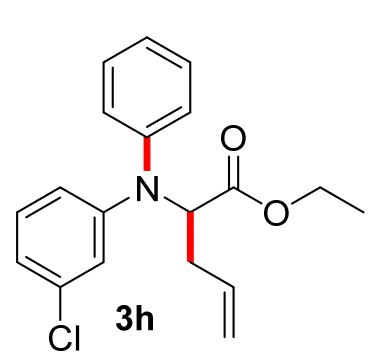

Following the general procedure, treatment of ethyl $\mathrm{N}$-allyl- $\mathrm{N}$-(3chlorophenyl)glycinate $\quad \mathbf{1 h} \quad(0.126 \quad \mathrm{~g}, \quad 0.5 \quad \mathrm{mmol})$ and 2(trimethylsilyl)phenyl trifluoromethane sulfonate $\mathbf{2 a}(0.223 \mathrm{~g}, 182 \mu \mathrm{L}$, $0.75 \mathrm{mmol})$ in the presence of $\mathrm{KF}(0.087 \mathrm{~g}, 1.5 \mathrm{mmol})$ and 18-crown-6 $(0.396 \mathrm{~g}, 1.5 \mathrm{mmol})$ in THF $(2.0 \mathrm{~mL})$ at $30{ }^{\circ} \mathrm{C}$ for $12 \mathrm{~h}$ followed by flash column chromatography (Pet. ether $/ \mathrm{EtOAc}=99 / 01)$ of the crude reaction mixture using silica gel afforded ethyl 2-((3-chlorophenyl)(phenyl)amino)pent-4-enoate $\mathbf{3 h}$ as yellow oil (0.119 g, 72\% yield).

$\boldsymbol{R}_{\mathbf{f}}\left(\right.$ Pet. ether $/$ EtOAc $=$ 95/05): 0.54; ${ }^{1} \mathbf{H}$ NMR (400 $\left.\mathbf{M H z}, \mathbf{C D C l}_{3}\right) \delta 7.36(\mathrm{t}, J=7.7 \mathrm{~Hz}, 2 \mathrm{H})$, 7.19 - $7.10(\mathrm{~m}, 3 \mathrm{H}), 6.87$ - $6.81(\mathrm{~m}, 2 \mathrm{H}), 6.71$ - $6.69(\mathrm{~m}, 1 \mathrm{H}), 5.83$ - $5.77(\mathrm{~m}, 1 \mathrm{H}), 5.14$ - $5.04(\mathrm{~m}$, 2H), $4.61(\mathrm{t}, J=7.5 \mathrm{~Hz}, 1 \mathrm{H}), 4.28-4.15(\mathrm{~m}, 2 \mathrm{H}), 2.69-2.57(\mathrm{~m}, 2 \mathrm{H}), 1.29-1.24(\mathrm{~m}, 4 \mathrm{H}) .{ }^{13} \mathrm{C}$

NMR (100 MHz, $\left.\mathbf{C D C l}_{3}\right) \delta 172.41,148.91,144.96,134.81,134.25,129.99,129.56,126.36$, 124.86, 120.39, 119.08, 118.01, 117.13, 62.73, 61.34, 34.44, 14.30. HRMS (ESI) calculated $[\mathrm{M}+\mathrm{H}]^{+}$for $\mathrm{C}_{19} \mathrm{H}_{21} \mathrm{O}_{2} \mathrm{NCl}: 330.1255$, found: 330.1255. FTIR (cm $\left.{ }^{-1}\right)$ 3016, 2925, 2401, 1691, $1640,1587,1396,1217,1032,1009,918,841,771$.

\section{Ethyl-2-((3-bromophenyl)(phenyl)amino)pent-4-enoate (3i)}<smiles>C=CCC(C(=O)OCC)N(c1ccccc1)c1cccc(Br)c1</smiles>

Following the general procedure, treatment of ethyl $\mathrm{N}$-allyl- $\mathrm{N}-(3-$ bromophenyl)glycinate $\mathbf{1 i}(0.149 \mathrm{~g}, \quad 0.5 \mathrm{mmol})$ and 2(trimethylsilyl)phenyl trifluoromethane sulfonate $\mathbf{2 a}(0.223 \mathrm{~g}, 182$ $\mu \mathrm{L}, 0.75 \mathrm{mmol})$ in the presence of $\mathrm{KF}(0.087 \mathrm{~g}, 1.5 \mathrm{mmol})$ and 18 crown-6 (0.396 g, $1.5 \mathrm{mmol})$ in THF $(2.0 \mathrm{~mL})$ at $30{ }^{\circ} \mathrm{C}$ for $12 \mathrm{~h}$ 
followed by flash column chromatography (Pet. ether $/$ EtOAc $=99 / 01)$ of the crude reaction mixture using silica gel afforded ethyl-2-((3-bromophenyl)(phenyl)amino)pent-4-enoate $\mathbf{3 i}$ as yellow oil $(0.107 \mathrm{~g}, 57 \%$ yield $)$.

$\boldsymbol{R}_{\mathbf{f}}($ Pet. ether $/$ EtOAc $=95 / 05): 0.45 ;{ }^{1} \mathbf{H}$ NMR $\left(\mathbf{4 0 0} \mathbf{~ M H z}, \mathbf{C D C l}_{3}\right) \delta 7.38(\mathrm{t}, J=7.7 \mathrm{~Hz}, 2 \mathrm{H})$, $7.21-7.17(\mathrm{~m}, 3 \mathrm{H}), 7.11-7.01(\mathrm{~m}, 3 \mathrm{H}), 6.77(\mathrm{~d}, J=7.3 \mathrm{~Hz}, 1 \mathrm{H}), 5.87-5.77(\mathrm{~m}, 1 \mathrm{H}), 5.12$ $5.06(\mathrm{~m}, 2 \mathrm{H}), 4.63(\mathrm{t}, J=7.5 \mathrm{~Hz}, 1 \mathrm{H}), 4.26-4.22(\mathrm{~m}, 2 \mathrm{H}), 2.68-2.62(\mathrm{~m}, 2 \mathrm{H}), 1.29(\mathrm{t}, J=7.1$ $\mathrm{Hz}, 3 \mathrm{H}) .{ }^{13} \mathbf{C}$ NMR (100 MHz, $\left.\mathbf{C D C l}_{3}\right) \delta 172.39,149.01,144.95,134.22,130.28,129.55$, $126.20,124.80,123.39,123.00,122.08,118.03,117.76,62.72,61.35,34.42,14.32$. HRMS (ESI) calculated $[\mathrm{M}+\mathrm{H}]{ }^{+}$for $\mathrm{C}_{19} \mathrm{H}_{21} \mathrm{O}_{2} \mathrm{NBr}$ : 374.0750, found: 374.0751. FTIR ( $\left.\mathbf{c m}^{-1}\right)$ 3023, 2936, 1728, 1588, 1523, 1451, 1369, 1321, 1258, 1108, 996.

\section{Ethyl-2-((3,4-dimethoxyphenyl)(phenyl)amino)pent-4-enoate (3j)}

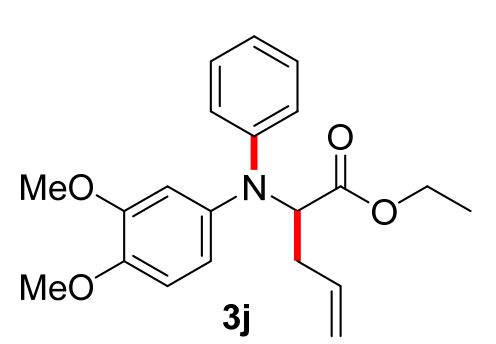

Following the general procedure, treatment of ethyl $\mathrm{N}$-allyl-N-(3,4dimethoxyphenyl)glycinate $\mathbf{1 j}(0.140 \mathrm{~g}, \quad 0.5 \mathrm{mmol})$ and 2 (trimethylsilyl)phenyl trifluoromethane sulfonate 2a $(0.223 \mathrm{~g}, 182$ $\mu \mathrm{L}, 0.75 \mathrm{mmol})$ in the presence of $\mathrm{KF}(0.087 \mathrm{~g}, 1.5 \mathrm{mmol})$ and 18-crown-6 $(0.396 \mathrm{~g}, 1.5 \mathrm{mmol})$ in THF $(2.0 \mathrm{~mL})$ at $30^{\circ} \mathrm{C}$ for 12 $\mathrm{h}$ followed by flash column chromatography $($ Pet. ether $/$ EtOAc $=99 / 01)$ of the crude reaction mixture using silica gel afforded ethyl-2-((3,4-dimethoxyphenyl)(phenyl)amino)pent-4-enoate $\mathbf{3 j}$ as yellow oil ( $0.142 \mathrm{~g}, 85 \%$ yield $)$.

$\boldsymbol{R}_{\mathbf{f}}\left(\right.$ Pet. ether $/$ EtOAc $=$ 95/05): 0.21; ${ }^{1} \mathbf{H}$ NMR $\left(\mathbf{4 0 0} \mathbf{~ M H z}, \mathbf{C D C l}_{3}\right) \delta 7.20(\mathrm{t}, J=7.6 \mathrm{~Hz}, 2 \mathrm{H})$, 6.92 - $6.86(\mathrm{~m}, 3 \mathrm{H}), 6.82(\mathrm{t}, J=7.1 \mathrm{~Hz}, 1 \mathrm{H}), 6.72(\mathrm{~d}, J=8.1 \mathrm{~Hz}, 2 \mathrm{H}), 5.88-5.81$ (m, 1H), 5.11 $5.07(\mathrm{~m}, 2 \mathrm{H}), 4.65(\mathrm{t}, J=7.4 \mathrm{~Hz}, 1 \mathrm{H}), 4.25-4.21(\mathrm{~m}, 2 \mathrm{H}), 3.93(\mathrm{~s}, 3 \mathrm{H}), 3.85(\mathrm{~s}, 3 \mathrm{H}), 2.63(\mathrm{t}, J=$ $6.9 \mathrm{~Hz}, 2 \mathrm{H}), 1.29(\mathrm{t}, J=7.1 \mathrm{~Hz}, 3 \mathrm{H}) .{ }^{13} \mathbf{C}$ NMR (100 MHz, $\left.\mathbf{C D C l}_{3}\right) \delta 172.89,149.42,148.42$, 147.12, 137.47, 134.59, 128.93, 121.04, 118.71, 117.57, 115.91, 112.69, 111.38, 62.21, 61.00, 55.99, 55.86, 34.73, 14.29. HRMS (ESI) calculated $[\mathrm{M}+\mathrm{H}]^{+}$for $\mathrm{C}_{21} \mathrm{H}_{26} \mathrm{O}_{4} \mathrm{~N}$ : 356.1856 , found: 356.1852. FTIR (cm $\left.{ }^{-1}\right)$ 3025, 2928, 1716, 1598, 1515, 1453, 1361, 1321, 1272, 1108, 998. 


\section{Ethyl-2-((3,4-difluorophenyl)(phenyl)amino)pent-4-enoate (3k)}

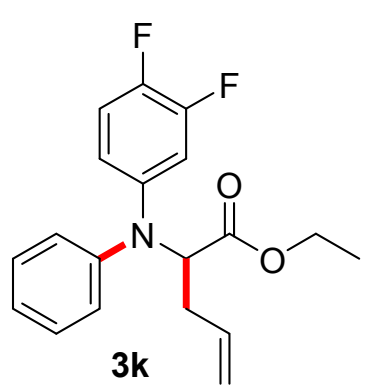

Following the general procedure, treatment of ethyl ethyl $N$-allyl- $N-(3,4-$ difluorophenyl)glycinate $\quad \mathbf{1 k} \quad\left(\begin{array}{lllll}0.128 & \mathrm{~g}, & 0.5 & \mathrm{mmol}\end{array}\right)$ and $\quad 2-$ (trimethylsilyl)phenyl trifluoromethane sulfonate $2 \mathrm{a}(0.223 \mathrm{~g}, 182 \mu \mathrm{L}$, $0.75 \mathrm{mmol})$ in the presence of $\mathrm{KF}(0.087 \mathrm{~g}, 1.5 \mathrm{mmol})$ and 18 -crown- 6 $(0.396 \mathrm{~g}, 1.5 \mathrm{mmol})$ in $\mathrm{THF}(2.0 \mathrm{~mL})$ at $30^{\circ} \mathrm{C}$ for $12 \mathrm{~h}$ followed by flash column chromatography (Pet. ether $/$ EtOAc $=99 / 01)$ of the crude reaction mixture using silica gel afforded ethyl-2-((3,4-difluorophenyl)(phenyl)amino)pent-4enoate $3 \mathbf{k}$ as yellow oil $(0.138 \mathrm{~g}, 83 \%$ yield).

$\boldsymbol{R}_{\mathbf{f}}($ Pet. ether $/$ EtOAc $=95 / 05): 0.47 ;{ }^{1} \mathbf{H}$ NMR (400 MHz, $\left.\mathbf{C D C l}_{3}\right) \delta$ 7.34-7.28 (m, 2H), $7.11-$ $6.93(\mathrm{~m}, 4 \mathrm{H}), 6.86-6.81(\mathrm{~m}, 1 \mathrm{H}), 6.71-6.69(\mathrm{~m}, 1 \mathrm{H}), 5.87-5.76(\mathrm{~m}, 1 \mathrm{H}), 5.09(\mathrm{t}, J=14.2 \mathrm{~Hz}$, $2 \mathrm{H}), 4.62-4.49(\mathrm{~m}, 1 \mathrm{H}), 4.25-4.22(\mathrm{~m}, 2 \mathrm{H}), 2.67-2.61(\mathrm{~m}, 2 \mathrm{H}), 1.28(\mathrm{t}, J=7.1 \mathrm{~Hz}, 3 \mathrm{H}) .{ }^{13} \mathbf{C}$ NMR (100 MHz, $\left.\mathbf{C D C l}_{3}\right) \delta 172.38,151.70\left(\mathrm{dd}, J_{1}=13.4, J_{2}=246.89\right), 147.24\left(\mathrm{dd}, J_{1}=13.5, J_{2}=\right.$ 242.84), 145.90, 143.59, 134.08, 129.38, 123.21, 117.96, 117.18 (d, $J=17.97), 111.31$ (d, $J=$ 19.66), 62.72, 61.27, 34.37, 14.20. HRMS (ESI) calculated $[\mathrm{M}+\mathrm{H}]{ }^{+}$for $\mathrm{C}_{19} \mathrm{H}_{20} \mathrm{O}_{2} \mathrm{NF}_{2}$ : 332.1457, found: 332.1452. FTIR ( cm $\left.^{-1}\right)$ 3060, 2935, 2360, 1746, 1642, 1611, 1513, 1454, 1097, $958,921,816$.

Ethyl 2-((3-chloro-4-fluorophenyl)(phenyl)amino)pent-4-enoate (3I)

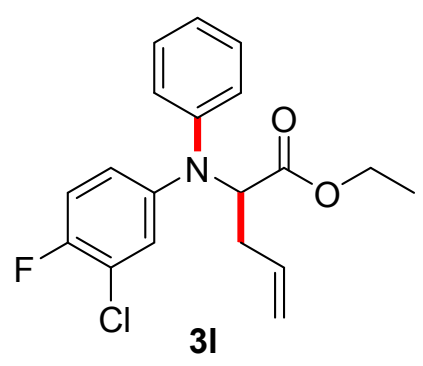

Following the general procedure, treatment of ethyl $\mathrm{N}$-allyl- $\mathrm{N}-(3-$ chloro-4-fluorophenyl)glycinate1l (0.135 g, $0.5 \mathrm{mmol})$ and 2(trimethylsilyl)phenyl trifluoromethane sulfonate 2a $(0.223 \mathrm{~g}, 182$ $\mu \mathrm{L}, 0.75 \mathrm{mmol})$ in the presence of $\mathrm{KF}(0.087 \mathrm{~g}, 1.5 \mathrm{mmol})$ and 18 crown-6 $(0.396 \mathrm{~g}, 1.5 \mathrm{mmol})$ in THF $(2.0 \mathrm{~mL})$ at $30^{\circ} \mathrm{C}$ for $12 \mathrm{~h}$ followed by flash column chromatography (Pet. ether /EtOAc $=$ 99/01) of the crude reaction mixture using silica gel afforded ethyl $(R)-2$-((3-chloro-4fluorophenyl)(phenyl)amino)pent-4-enoate 31 as yellow oil (0.133 g, 77\% yield).

$\boldsymbol{R}_{\mathbf{f}}($ Pet. ether $/$ EtOAc $=95 / 05): 0.46 ;{ }^{1} \mathbf{H}$ NMR $\left(\mathbf{4 0 0} \mathbf{~ M H z}, \mathbf{C D C l}_{3}\right) \delta 7.30(\mathrm{t}, J=7.7 \mathrm{~Hz}, 2 \mathrm{H})$, 7.11 - 6.88 (m, 6H), 5.84 - 5.77 (m, 1H), 5.12 - $5.04(\mathrm{~m}, 2 \mathrm{H}), 4.60$ (t, J= 7.5 Hz, 1H), 4.27 - 4.16 (m, 2H), 2.69-2.56 (m, 2H), 1.30 - $1.25(\mathrm{~m}, 3 \mathrm{H}) .{ }^{13} \mathbf{C}$ NMR (100 MHz, $\left.\mathbf{C D C l}_{3}\right) \delta$ 172.43, 154.157 (d, J = 248.1 Hz), 146.27, 143.41, 143.38, 134.14, 129.54, 129.43, 125.21, 122.93 (d, $J$ 
$=6.6 \mathrm{~Hz}), 122.74,122.26,121.25,121.07,120.77,118.07,116.86,116.64,114.57,62.77,61.35$, 34.46, 14.30. HRMS (ESI) calculated $[\mathrm{M}+\mathrm{H}]^{+}$for $\mathrm{C}_{19} \mathrm{H}_{20} \mathrm{O}_{2} \mathrm{NClF}: 348.1161$, found: 348.1159 . FTIR $\left(\mathbf{c m}^{-1}\right)$ 3018, 2929, 2401, 1733, 1641, 1596, 1498, 1371, 1217, 1129, 993, 824, 772.

\section{Ethyl 2-(phenyl(3,4,5-trimethoxyphenyl)amino)pent-4-enoate (3m)}<smiles>C=CCC(C(=O)OCC)N(c1ccccc1)c1cc(OC)c(OC)c(OC)c1</smiles>

Following the general procedure, treatment of ethyl $N$-allyl- $N$ (3,4,5-trimethoxyphenyl)glycinate $1 \mathrm{~m}(0.154 \mathrm{~g}, 0.5 \mathrm{mmol})$ and 2(trimethylsilyl)phenyl trifluoromethane sulfonate 2a (0.223 g, 182 $\mu \mathrm{L}, 0.75 \mathrm{mmol})$ in the presence of $\mathrm{KF}(0.087 \mathrm{~g}, 1.5 \mathrm{mmol})$ and 18 crown-6 (0.396 g, $1.5 \mathrm{mmol})$ in THF $(2.0 \mathrm{~mL})$ at $30{ }^{\circ} \mathrm{C}$ for $12 \mathrm{~h}$ followed by flash column chromatography (Pet. ether $/$ EtOAc $=99 / 01)$ of the crude reaction mixture using silica gel afforded Ethyl 2-(phenyl(3,4,5-trimethoxyphenyl)amino)pent-4-enoate $3 \mathrm{~m}$ as yellow oil $(0.125 \mathrm{~g}, 65 \%$ yield).

$\boldsymbol{R}_{\mathbf{f}}\left(\right.$ Pet. ether $/$ EtOAc $=$ 95/05): 0.41; ${ }^{1} \mathbf{H}$ NMR (400 $\left.\mathbf{M H z}, \mathbf{C D C l}_{3}\right) \delta 7.25(\mathrm{t}, J=7.8 \mathrm{~Hz}, 2 \mathrm{H})$, $6.94-6.86(\mathrm{~m}, 3 \mathrm{H}), 6.45(\mathrm{~s}, 2 \mathrm{H}), 5.90-5.80(\mathrm{~m}, 1 \mathrm{H}), 5.12-5.08(\mathrm{~m}, 2 \mathrm{H}), 4.64(\mathrm{t}, J=7.5 \mathrm{~Hz}$, 1H), 4.29 - $4.17(\mathrm{~m}, 2 \mathrm{H}), 3.89(\mathrm{~s}, 3 \mathrm{H}), 3.80(\mathrm{~s}, 6 \mathrm{H}), 2.67-2.64(\mathrm{~m}, 2 \mathrm{H}), 1.29(\mathrm{t}, J=6.8 \mathrm{~Hz}, 3 \mathrm{H})$.

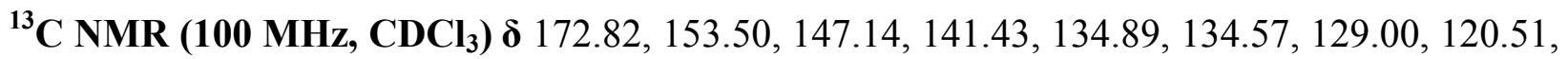
119.10, 117.61, 103.30, 62.47, 61.04, 60.94, 55.96, 34.59, 14.26. HRMS (ESI) calculated $[\mathrm{M}+\mathrm{Na}]^{+}$for $\mathrm{C}_{21} \mathrm{H}_{27} \mathrm{O}_{5} \mathrm{NNa}$ : 408.1781, found: 408.1777. FTIR (cm $\left.{ }^{-1}\right)$ 3014, 2938, 2836, 2250, $1640,1590,1495,1462,1369,1229,1007,856,747$.

\section{2-(Diphenylamino)pent-4-enenitrile (3n)}

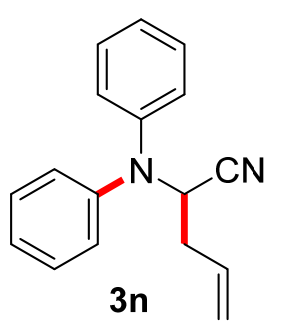

Following the general procedure, treatment of 2-(allyl(phenyl)amino) acetonitrile $1 \mathrm{n} \quad(0.086 \mathrm{~g}, \quad 0.5 \mathrm{mmol})$ and 2-(trimethylsilyl)phenyl trifluoromethane sulfonate $2 \mathbf{a}(0.223 \mathrm{~g}, 182 \mu \mathrm{L}, 0.75 \mathrm{mmol})$ in the presence of $\mathrm{KF}(0.087 \mathrm{~g}, 1.5 \mathrm{mmol})$ and 18-crown-6 (0.396 g, $1.5 \mathrm{mmol})$ in THF $(2.0$ $\mathrm{mL}$ ) at $\mathrm{rt}$ for $12 \mathrm{~h}$ followed by flash column chromatography (Pet. ether $/$ EtOAc $=99 / 01)$ of the crude reaction mixture using silica gel afforded 2-(diphenylamino)pent4-enenitrile 3n as yellow oil (0.099 g, 80\% yield).

$\boldsymbol{R}_{\mathbf{f}}($ Pet. ether $/$ EtOAc $=95 / 05): 0.52 ;{ }^{1} \mathbf{H}$ NMR (400 MHz, $\left.\mathbf{C D C l}_{3}\right) \delta 7.39(\mathrm{t}, J=7.8 \mathrm{~Hz}, 4 \mathrm{H})$, $7.18(\mathrm{t}, J=7.3 \mathrm{~Hz}, 2 \mathrm{H}), 7.10(\mathrm{~d}, J=8.0 \mathrm{~Hz}, 4 \mathrm{H}), 5.94-5.84(\mathrm{~m}, 1 \mathrm{H}), 5.27(\mathrm{dd}, J=13.5,7.5 \mathrm{~Hz}$, 
2H), $4.86(\mathrm{t}, J=5.4 \mathrm{~Hz}, 1 \mathrm{H}), 2.72-2.49(\mathrm{~m}, 2 \mathrm{H}) .{ }^{13} \mathbf{C}$ NMR (100 MHz, CDCl $\left.\mathbf{3}\right) \delta 145.73$, 131.73, 129.67, 124.07, 123.20, 120.02, 118.60, 51.28, 36.59. HRMS (ESI) calculated [M+H] ${ }^{+}$ for $\mathrm{C}_{17} \mathrm{H}_{17} \mathrm{~N}_{2}$ : 249.1386, found: 249.1382 . FTIR (cm $\left.{ }^{-1}\right)$ 3019, 2948, 1719, 11604, 1446, 1342 , $1291,1086,963,760$.

Methyl-2-(diphenylamino)pent-4-enoate (3o)

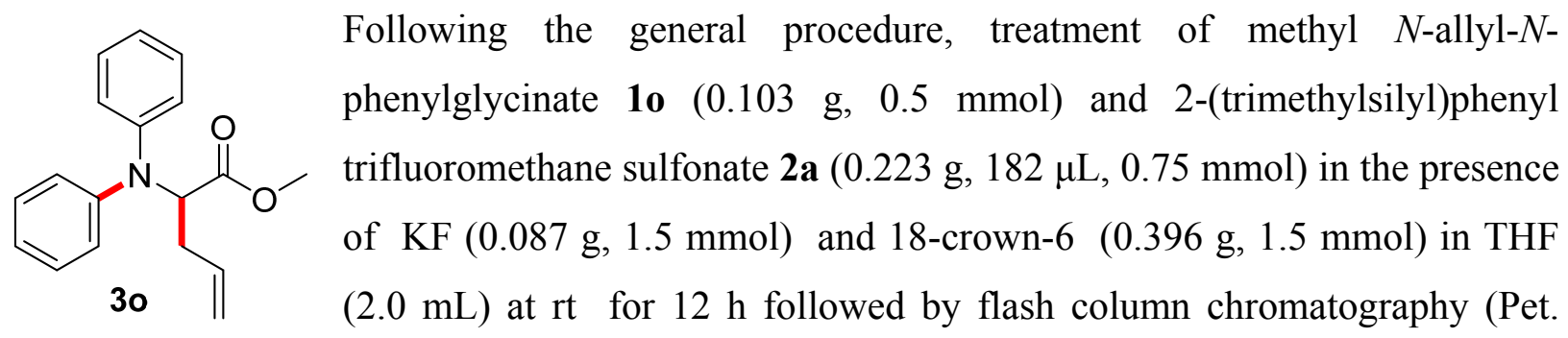
ether $/$ EtOAc $=99 / 01)$ of the crude reaction mixture using silica gel afforded methyl-2(diphenylamino)pent-4-enoate $\mathbf{3 o}$ as yellow oil (0.111 g, 79\% yield).

$\boldsymbol{R}_{\mathbf{f}}\left(\right.$ Pet. ether $/$ EtOAc $=$ 95/05): 0.56; ${ }^{1} \mathbf{H}$ NMR (400 $\left.\mathbf{M H z}, \mathbf{C D C l}_{3}\right) \delta 7.33(\mathrm{t}, J=7.8 \mathrm{~Hz}, 4 \mathrm{H})$, 7.08-7.04 ( $\mathrm{m}, J=9.4 \mathrm{~Hz}, 6 \mathrm{H}), 5.92-5.82(\mathrm{~m}, 1 \mathrm{H}), 5.22-5.00(\mathrm{~m}, 2 \mathrm{H}), 4.73(\mathrm{t}, J=7.5 \mathrm{~Hz}, 1 \mathrm{H})$, 3.77 (s, 3H), $2.71(\mathrm{t}, J=7.1 \mathrm{~Hz}, 2 \mathrm{H}) .{ }^{13} \mathbf{C}$ NMR (100 MHz, CDCl $) \delta 173.28,146.54,134.44$, 129.24, 122.58, 122.36, 117.77, 62.51, 52.06, 34.39.HRMS (ESI) calculated $[\mathrm{M}+\mathrm{H}]{ }^{+}$for $\mathrm{C}_{18} \mathrm{H}_{20} \mathrm{O}_{2} \mathrm{~N}$ : 282.1489, found: 282.1487. FTIR (cm $\left.{ }^{-1}\right)$ 3019, 2951, 1722, 1598, 1577, 1499, 1409, 1344, 1297, 1249, 1216, 1099, 995, 759.

\section{Benzyl 2-(diphenylamino)pent-4-enoate (3p)}

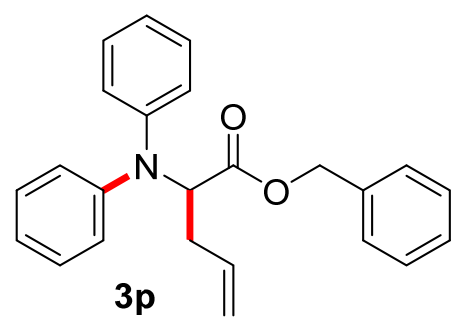

Following the general procedure, treatment of ethyl benzyl $\mathrm{N}$-allyl$N$-phenylglycinate $\quad \mathbf{1 p} \quad(0.141 \quad \mathrm{~g}, \quad 0.5 \quad \mathrm{mmol})$ and 2(trimethylsilyl)phenyl trifluoromethane sulfonate $\mathbf{2 a}(0.223 \mathrm{~g}, 182$ $\mu \mathrm{L}, 0.75 \mathrm{mmol})$ in the presence of $\mathrm{KF}(0.087 \mathrm{~g}, 1.5 \mathrm{mmol})$ and 18-crown-6 (0.396 g, $1.5 \mathrm{mmol})$ in THF $(2.0 \mathrm{~mL})$ at $\mathrm{rt}$ for $12 \mathrm{~h}$ followed by flash column chromatography $($ Pet. ether $/$ EtOAc $=99 / 01)$ of the crude reaction mixture using silica gel afforded benzyl 2-(diphenylamino)pent-4-enoate 3p as yellow oil (0.124 g, 84\% yield).

$\boldsymbol{R}_{\mathbf{f}}($ Pet. ether $/$ EtOAc $=95 / 05)$ : 0.55; ${ }^{1} \mathbf{H}$ NMR (400 MHz, $\left.\mathbf{C D C l}_{3}\right) \delta 7.47$ - $7.24(\mathrm{~m}, 9 \mathrm{H}), 7.12$ $6.95(\mathrm{~m}, 6 \mathrm{H}), 5.98-5.76(\mathrm{~m}, 1 \mathrm{H}), 5.24(\mathrm{q}, J=12.3 \mathrm{~Hz}, 2 \mathrm{H}), 5.17-5.05(\mathrm{~m}, 2 \mathrm{H}), 4.80(\mathrm{t}, J=7.5$ 
$\mathrm{Hz}, 1 \mathrm{H}), 2.73(\mathrm{~d}, J=2.9 \mathrm{~Hz}, 2 \mathrm{H}) .{ }^{13} \mathbf{C}$ NMR (100 MHz, $\mathbf{C D C l}_{3} \delta 172.66,146.56,135.60$, $134.41,129.23,128.56,128.37,128.31,122.72,122.38,117.85,66.90,62.65,34.48$. HRMS (ESI) calculated $[\mathrm{M}+\mathrm{H}]^{+}$for $\mathrm{C}_{24} \mathrm{H}_{24} \mathrm{O}_{2} \mathrm{~N}$ : 358.1802, found: 358.1800. FTIR ( $\left.\mathbf{c m}^{-1}\right)$ 3018, 2982, 1732, 1641, 1565, 1440, 1371, 1216, 1028, 989, 854, 772.

\section{Tert-butyl 2-(diphenylamino)pent-4-enoate (3q)}

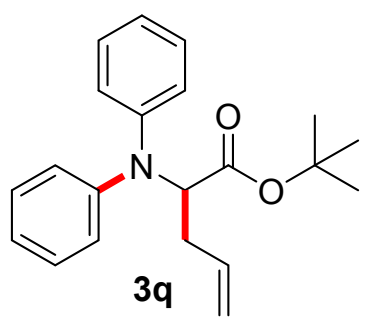

Following the general procedure, treatment of tert-butyl $\mathrm{N}$-allyl- $\mathrm{N}$ phenylglycinate 1q $(0.124 \mathrm{~g}, 0.5 \mathrm{mmol})$ and 2-(trimethylsilyl)phenyl trifluoromethane sulfonate $2 \mathrm{a}(0.223 \mathrm{~g}, 182 \mu \mathrm{L}, 0.75 \mathrm{mmol})$ in the presence of $\mathrm{KF}(0.087 \mathrm{~g}, 1.5 \mathrm{mmol})$ and 18-crown-6 (0.396 g, 1.5 $\mathrm{mmol})$ in THF $(2.0 \mathrm{~mL})$ at $\mathrm{rt}$ for $12 \mathrm{~h}$ followed by flash column chromatography $($ Pet. ether $/$ EtOAc $=99 / 01)$ of the crude reaction mixture using silica gel afforded tert-butyl 2-(diphenylamino)pent-4-enoate $3 q$ as yellow oil ( $0.133 \mathrm{~g}, 82 \%$ yield).

$\boldsymbol{R}_{\mathbf{f}}\left(\right.$ Pet. ether $/$ EtOAc $=$ 95/05): 0.53; ${ }^{1} \mathbf{H}$ NMR $\left(\mathbf{4 0 0} \mathbf{~ M H z}, \mathbf{C D C l}_{3}\right) \delta 7.29(\mathrm{t}, J=7.7 \mathrm{~Hz}, 4 \mathrm{H})$, $7.04-7.01(\mathrm{~m}, 6 \mathrm{H}), 5.90-5.80(\mathrm{~m}, 1 \mathrm{H}), 5.10-5.05(\mathrm{~m}, 2 \mathrm{H}), 4.56(\mathrm{t}, J=7.5 \mathrm{~Hz}, 1 \mathrm{H}), 2.65(\mathrm{t}, J=$ $7.1 \mathrm{~Hz}, 2 \mathrm{H}), 1.45$ (s, 9H). ${ }^{13} \mathbf{C}$ NMR (100 MHz, $\left.\mathbf{C D C l}_{3}\right) \delta$ 172.00, 146.92, 134.90, 129.13, 122.73, 122.15, 117.53, 81.78, 63.63, 34.65, 28.11. HRMS (ESI) calculated $[\mathrm{M}+\mathrm{H}]{ }^{+}$for $\mathrm{C}_{21} \mathrm{H}_{26} \mathrm{O}_{2} \mathrm{~N}: 324.1958$, found: 324.1953. FTIR (cm $\left.{ }^{-1}\right)$ 3014, 2981, 1598, 1734, 1640, 1590, 1495, 1462, 1431, 1415, 1369, 1291, 921.

\section{2-(Diphenylamino)-1-phenylpent-4-en-1-one (3r)}

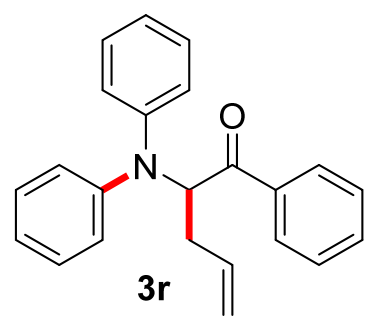

Following the general procedure, treatment of 2-(allyl(phenyl)amino)-1phenylethan-1-one 1r (0.126 g, $0.5 \mathrm{mmol})$ and 2-(trimethylsilyl)phenyl trifluoromethane sulfonate $2 \mathrm{a}(0.223 \mathrm{~g}, 182 \mu \mathrm{L}, 0.75 \mathrm{mmol})$ in the presence of $\mathrm{KF}(0.087 \mathrm{~g}, 1.5 \mathrm{mmol})$ and 18-crown-6 (0.396 g, 1.5 $\mathrm{mmol})$ in THF $(2.0 \mathrm{~mL})$ at $\mathrm{rt}$ for $12 \mathrm{~h}$ followed by flash column chromatography (Pet. ether $/$ EtOAc $=99 / 01)$ of the crude reaction mixture using silica gel afforded 2-(diphenylamino)-1-phenylpent-4-en-1-one $3 \mathbf{r}$ as yellow solid ( $0.149 \mathrm{~g}, 91 \%$ yield). $\boldsymbol{R}_{\mathbf{f}}($ Pet. ether $/$ EtOAc $=95 / 05): 0.50 ;{ }^{1} \mathbf{H}$ NMR $\left(400 \mathbf{~ M H z}, \mathbf{C D C l}_{3}\right) \delta 8.00(\mathrm{~d}, J=7.7 \mathrm{~Hz}, 2 \mathrm{H})$, 7.63 (t, $J=7.3 \mathrm{~Hz}, 1 \mathrm{H}), 7.49$ (t, $J=7.6 \mathrm{~Hz}, 2 \mathrm{H}), 7.33$ (t, $J=7.7 \mathrm{~Hz}, 4 \mathrm{H}), 7.10$ (t, $J=7.3 \mathrm{~Hz}$, 2H), $6.96(\mathrm{~d}, J=8.0 \mathrm{~Hz}, 4 \mathrm{H}), 6.04-5.94(\mathrm{~m}, 1 \mathrm{H}), 5.61(\mathrm{t}, J=6.2 \mathrm{~Hz}, 1 \mathrm{H}), 5.28(\mathrm{~d}, J=17.1 \mathrm{~Hz}$, 
1H), $5.19(\mathrm{~d}, J=10.1 \mathrm{~Hz}, 1 \mathrm{H}), 3.06-2.99(\mathrm{~m}, 1 \mathrm{H}), 2.75-2.69(\mathrm{~m}, 1 \mathrm{H}) .{ }^{13} \mathbf{C}$ NMR (100 MHz, $\left.\mathbf{C D C l}_{3}\right) \delta 197.76,146.12,136.59,135.20,133.27,129.46,128.77,128.60,122.68,117.68$, 63.29, 32.71. HRMS (ESI) calculated $[\mathrm{M}+\mathrm{H}]^{+}$for $\mathrm{C}_{23} \mathrm{H}_{22} \mathrm{ON}: 328.1696$, found: 328.1694 . FTIR (cm $\left.{ }^{-1}\right)$ 3019, 2947, 1721, 1593, 1495, 1449, 1356, 1274, 1132, 1036, 909, 760.

\section{2-(Diphenylamino)-1-(p-tolyl)pent-4-en-1-one (3s)}

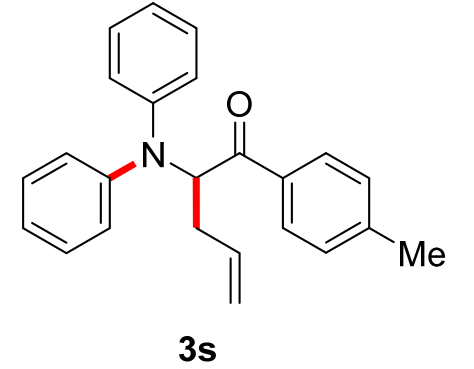

99/01) of the crude reaction mixture using silica gel afforded 2-(diphenylamino)-1-( $p$ tolyl)pent-4-en-1-one 3s as yellow oil ( $0.145 \mathrm{~g}, 85 \%$ yield).

$\boldsymbol{R}_{\mathbf{f}}($ Pet. ether $/$ EtOAc $=95 / 05): 0.35 ;{ }^{1} \mathbf{H}$ NMR (400 $\left.\mathbf{M H z}, \mathbf{C D C l}_{3}\right) \delta 7.90(\mathrm{~d}, J=8.1 \mathrm{~Hz}, 2 \mathrm{H})$, $7.32-7.25(\mathrm{~m}, 6 \mathrm{H}), 7.06(\mathrm{t}, J=7.3 \mathrm{~Hz}, 2 \mathrm{H}), 6.95(\mathrm{~d}, J=7.9 \mathrm{~Hz}, 4 \mathrm{H}), 6.02-5.92(\mathrm{~m}, 1 \mathrm{H}), 5.58$ (t, $J=7.1 \mathrm{~Hz}, 1 \mathrm{H}), 5.23(\mathrm{dd}, J=37.7,13.7 \mathrm{~Hz}, 2 \mathrm{H}), 3.03-2.95(\mathrm{~m}, 1 \mathrm{H}), 2.72-2.65$ (m, 1H), 2.44 (s, 3H). ${ }^{13} \mathbf{C}$ NMR (100 MHz, $\left.\mathbf{C D C l}_{3}\right) \delta$ 197.23, 146.13, 144.05, 135.26, 134.01, 129.43, 129.41, 128.70, 122.66, 122.59, 117.53, 63.06, 32.74, 21.75. HRMS (ESI) calculated [M+H] ${ }^{+}$ for $\mathrm{C}_{24} \mathrm{H}_{24} \mathrm{ON}$ : 342.1852, found: 342.1851. FTIR (cm $\left.{ }^{-1}\right)$ 3024, 2921, 2348, 1716, 1578, 1513, $1451,1356,1313,1278,1108,995$.

\section{$N$-(1-(4-bromophenyl)but-3-en-1-yl)- $N$-phenylaniline (3t)}<smiles>C=CCC(C(=O)c1ccc(Br)cc1)N(c1ccccc1)c1ccccc1</smiles>

Following the general procedure, treatment of 2(allyl(phenyl)amino)-1-(4-bromophenyl)ethan-1-one 1t (0.165 g, 0.5 mmol) and 2-(trimethylsilyl)phenyl trifluoromethane sulfonate $\mathbf{2 a}$ $(0.223 \mathrm{~g}, 182 \mu \mathrm{L}, 0.75 \mathrm{mmol})$ in the presence of $\mathrm{KF}(0.087 \mathrm{~g}, 1.5$ mmol) and 18-crown-6 (0.396 g, $1.5 \mathrm{mmol})$ in THF $(2.0 \mathrm{~mL})$ at $\mathrm{rt}$ for $12 \mathrm{~h}$ followed by flash column chromatography (Pet. ether /EtOAc $=99 / 01)$ of the crude reaction mixture using silica gel afforded $N$-(1-(4-bromophenyl)but-3-en-1-yl)- $N$-phenylaniline 3t as yellow solid $(0.166 \mathrm{~g}, 88 \%$ yield $)$. 
$\boldsymbol{R}_{\mathbf{f}}($ Pet. ether $/$ EtOAc $=95 / 05): 0.51 ;{ }^{1} \mathbf{H}$ NMR (400 MHz, $\left.\mathbf{C D C l}_{3}\right) \delta 7.82(\mathrm{~d}, J=8.5 \mathrm{~Hz}, 2 \mathrm{H})$, 7.57 (d, $J=8.4 \mathrm{~Hz}, 2 \mathrm{H}), 7.29$ (t, $J=7.3 \mathrm{~Hz}, 4 \mathrm{H}), 7.07$ (t, $J=7.4 \mathrm{~Hz}, 2 \mathrm{H}), 6.91$ (d, $J=7.9 \mathrm{~Hz}$, 4H), $6.00-5.90(\mathrm{~m}, 1 \mathrm{H}), 5.50(\mathrm{dd}, J=7.8,5.8 \mathrm{~Hz}, 1 \mathrm{H}), 5.25(\mathrm{~d}, J=17.1 \mathrm{~Hz}, 1 \mathrm{H}), 5.16(\mathrm{~d}, J=$ $10.2 \mathrm{~Hz}, 1 \mathrm{H}), 3.02-2.95(\mathrm{~m}, 1 \mathrm{H}), 2.79-2.57$ (m, 1H). ${ }^{13} \mathbf{C}$ NMR (100 MHz, CDCl $) \delta 196.71$, $145.96,135.24,134.96,132.02,130.11,129.80,129.52,128.37,122.83,122.58,117.85,63.35$, 32.60. HRMS (ESI) calculated $[\mathrm{M}+\mathrm{H}]^{+}$for $\mathrm{C}_{23} \mathrm{H}_{21} \mathrm{ONBr}$ : 406.0801, found: 406.0803. FTIR $\left(\mathbf{c m}^{-1}\right)$ 3020, 2902, 1712, 1604, 1492, 1421, 1278, 1100, 1035, 1009, 931, 865, 759.

\section{Ethyl 2-((3,4-dimethylphenyl)(phenyl)amino)pent-4-enoate (3u)}

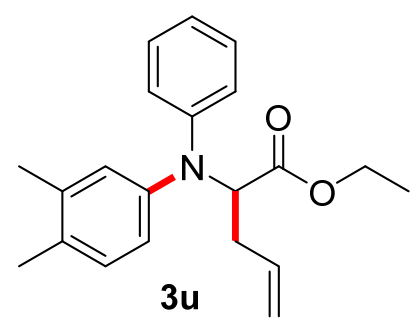

Following the general procedure, treatment of ethyl $\mathrm{N}$-allyl- $N$ phenylglycinate $1 \mathrm{a}(0.110 \mathrm{~g}, \quad 0.5 \mathrm{mmol})$ and 4,5-dimethyl-2(trimethylsilyl)phenyl trifluoromethanesulfonate $\mathbf{2 b}(0.245 \mathrm{~g}, 0.75$ $\mathrm{mmol})$ in the presence of $\mathrm{KF}(0.087 \mathrm{~g}, 1.5 \mathrm{mmol})$ and 18-crown-6 $(0.396 \mathrm{~g}, 1.5 \mathrm{mmol})$ in THF $(2.0 \mathrm{~mL})$ at $\mathrm{rt}$ for $12 \mathrm{~h}$ followed by flash column chromatography $($ Pet. ether $/$ EtOAc $=99 / 01)$ of the crude reaction mixture using silica gel afforded ethyl 2-((3,4-dimethylphenyl)(phenyl)amino)pent-4-enoate $\mathbf{3 u}$ as yellow oil (0.128 g, 79\% yield).

$\boldsymbol{R}_{\mathbf{f}}\left(\right.$ Pet. ether $/$ EtOAc $=$ 95/05): 0.56; ${ }^{1} \mathbf{H}$ NMR (400 $\left.\mathbf{M H z}, \mathbf{C D C l}_{3}\right) \delta 7.25(\mathrm{t}, J=7.8 \mathrm{~Hz}, 2 \mathrm{H})$, $7.13(\mathrm{~d}, J=8.0 \mathrm{~Hz}, 1 \mathrm{H}), 6.98(\mathrm{~s}, 1 \mathrm{H}), 6.95-6.83(\mathrm{~m}, 4 \mathrm{H}), 6.05-5.68(\mathrm{~m}, 1 \mathrm{H}), 5.12(\mathrm{~d}, J=12.4$ Hz, 2H), 4.66 (t, J=7.5 Hz, 1H), $4.32-4.16(\mathrm{~m}, 2 \mathrm{H}), 2.67$ (t, $J=7.1 \mathrm{~Hz}, 2 \mathrm{H}), 2.30$ (s, 3H), 2.27 (s, 3H), 1.29 (t, $J=7.1 \mathrm{~Hz}, 3 \mathrm{H}) .{ }^{13} \mathbf{C}$ NMR (100 MHz, $\left.\mathbf{C D C l}_{3}\right) \delta$ 172.92, 147.76, 143.44, $137.53,134.74,132.44,130.43,129.01,127.22,123.37,120.22,119.24,117.61,62.62,61.06$, 34.63, 20.08, 19.26, 14.32. HRMS (ESI) calculated $[\mathrm{M}+\mathrm{H}]^{+}$for $\mathrm{C}_{21} \mathrm{H}_{26} \mathrm{O}_{2} \mathrm{~N}: 324.1958$, found: 324.1953. FTIR (cm $\left.{ }^{-1}\right)$ 3020, 2404, 1795, 1726, 1596, 1495, 1444, 1334, 1282, 1216, 1036, 930, 760.

\section{Ethyl-2-(benzo[d][1,3]dioxol-5-yl(phenyl)amino)pent-4-enoate (3v)}

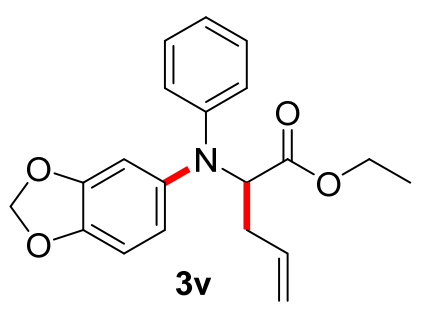

Following the general procedure, treatment of ethyl $\mathrm{N}$-allyl- $\mathrm{N}$ phenylglycinate 1a $(0.110 \quad \mathrm{~g}, \quad 0.5 \quad \mathrm{mmol})$ and 6(trimethylsilyl)benzo[d][1,3]dioxol-5-yl trifluoromethanesulfonate 2c $(0.257 \mathrm{~g}, 0.75 \mathrm{mmol})$ in the presence of $\mathrm{KF}(0.087 \mathrm{~g}, 1.5 \mathrm{mmol})$ and 
18-crown-6 $(0.396 \mathrm{~g}, 1.5 \mathrm{mmol})$ in THF $(2.0 \mathrm{~mL})$ at $30{ }^{\circ} \mathrm{C}$ for $12 \mathrm{~h}$ followed by flash column chromatography $($ Pet. ether $/$ EtOAc $=99 / 01)$ of the crude reaction mixture using silica gel afforded ethyl-2-(benzo[d][1,3]dioxol-5-yl(phenyl)amino)pent-4-enoate $\mathbf{3 v}$ as pale yellow oil (0.124 g, 73\% yield).

$\boldsymbol{R}_{\mathbf{f}}($ Pet. ether/EtOAc $=95 / 05): 0.45 ;{ }^{1} \mathbf{H}$ NMR $\left(\mathbf{4 0 0} \mathbf{~ M H z}, \mathbf{C D C l}_{3}\right) \delta 7.17(\mathrm{t}, J=7.9 \mathrm{~Hz}, 2 \mathrm{H})$, $6.82-6.78(\mathrm{~m}, 3 \mathrm{H}), 6.74-6.69(\mathrm{~m}, 3 \mathrm{H}), 5.98(\mathrm{~s}, 2 \mathrm{H}), 5.88-5.62(\mathrm{~m}, 1 \mathrm{H}), 5.08(\mathrm{~s}, 1 \mathrm{H}), 5.05(\mathrm{~d}$, $J=5.8 \mathrm{~Hz}, 1 \mathrm{H}), 4.57(\mathrm{t}, J=7.5 \mathrm{~Hz}, 1 \mathrm{H}), 4.21-4.17(\mathrm{~m}, 2 \mathrm{H}), 2.60$ (t, $J=7.1 \mathrm{~Hz}, 2 \mathrm{H}), 1.25(\mathrm{t}, J$ $=7.1 \mathrm{~Hz}, 3 \mathrm{H}) .{ }^{13} \mathbf{C}$ NMR (100 MHz, $\left.\mathbf{C D C l}_{3}\right) \delta 172.85,148.36,145.46,138.94,134.57,129.03$, $121.88,119.17,117.76,116.63,109.99,108.47,101.46,62.54,61.17,34.73,14.35$. HRMS (ESI) calculated $[\mathrm{M}+\mathrm{H}]^{+}$for $\mathrm{C}_{20} \mathrm{H}_{22} \mathrm{O}_{4} \mathrm{~N}: 340.1543$, found: 340.1530 . FTIR ( $\left.\mathbf{c m}^{-1}\right)$ 3018, 2983, 2776, 2402, 1733, 1597, 1459, 1217, 1040, 994, 926, 668.

\section{Ethyl 2-((3,4-difluorophenyl)(phenyl)amino)pent-4-enoate (3w)}

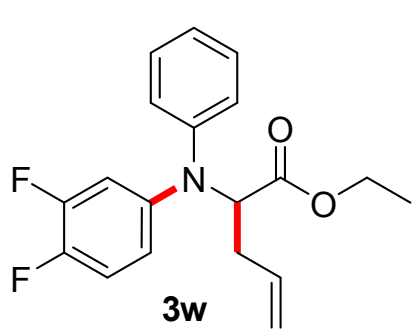

Following the general procedure, treatment of ethyl $\mathrm{N}$-allyl- $\mathrm{N}$ phenylglycinate $\mathbf{1 a}(0.110 \mathrm{~g}, \quad 0.5 \mathrm{mmol})$ and 4,5-difluoro-2(trimethylsilyl)phenyl trifluoromethanesulfonate $2 \mathbf{2 d}(0.251 \mathrm{~g}, 0.75$ $\mathrm{mmol})$ in the presence of $\mathrm{KF}(0.087 \mathrm{~g}, 1.5 \mathrm{mmol})$ and 18-crown-6

$(0.396 \mathrm{~g}, 1.5 \mathrm{mmol})$ in THF $(2.0 \mathrm{~mL})$ at $\mathrm{rt}$ for $12 \mathrm{~h}$ followed by flash column chromatography (Pet. ether $/ \mathrm{EtOAc}=99 / 01)$ of the crude reaction mixture using silica gel afforded ethyl 2-((3,4-difluorophenyl)(phenyl)amino)pent-4-enoate $\mathbf{3 w}$ as yellow oil (0.116 g, $70 \%$ yield).

$\boldsymbol{R}_{\mathbf{f}}($ Pet. ether $/$ EtOAc $=95 / 05): 0.46 ;{ }^{1} \mathbf{H}$ NMR $\left(\mathbf{4 0 0} \mathbf{~ M H z}, \mathbf{C D C l}_{3}\right) \delta 7.38-7.22(\mathrm{~m}, 2 \mathrm{H})$, 7.$6.94(\mathrm{~m}, 4 \mathrm{H}), 6.83-6.77 \mathrm{~m} \mathrm{1H}), 6.72-6.63(\mathrm{~m}, 1 \mathrm{H}), 5.83-5.73(\mathrm{~m}, 1 \mathrm{H}), 5.05(\mathrm{dd}, J=23.3,5.8$ $\mathrm{Hz}, 2 \mathrm{H}), 4.59-4.55(\mathrm{~m}, 1 \mathrm{H}), 4.27-4.13(\mathrm{~m}, 2 \mathrm{H}), 2.68-2.50(\mathrm{~m}, 2 \mathrm{H}), 1.25(\mathrm{t}, J=7.1 \mathrm{~Hz}, 3 \mathrm{H})$. ${ }^{13}$ C NMR (100 MHz, $\left.\mathbf{C D C l}_{3}\right) \delta 172.49,150.57$ (dd, $\left.J=144.5,13.8 \mathrm{~Hz}\right), 146.14$ (dd, $J=245.6$, $12.7 \mathrm{~Hz}$ ), 146.00, 143.63 (dd, $J=7.8,2.5 \mathrm{~Hz}), 134.18,129.48,123.29,118.07,117.83$ (dd, $J=$ 5.2, $2.8 \mathrm{~Hz}), 117.27$ (d, $J=17.4 \mathrm{~Hz}), 111.44$ (d, $19.4 \mathrm{~Hz}), 62.82,61.38,34.47,14.31$. HRMS (ESI) calculated $[\mathrm{M}+\mathrm{H}]{ }^{+}$for $\mathrm{C}_{19} \mathrm{H}_{20} \mathrm{O}_{2} \mathrm{NF}_{2}: 332.1457$, found: 332.1448. FTIR (cm ${ }^{-1}$ ) 3018, 2898, 1718, 1604, 1490, 1440, 1382, 1328, 1251, 1129, 1083, 940, 872. 


\section{Ethyl 2-(naphthalen-2-yl(phenyl)amino)pent-4-enoate (3x)}

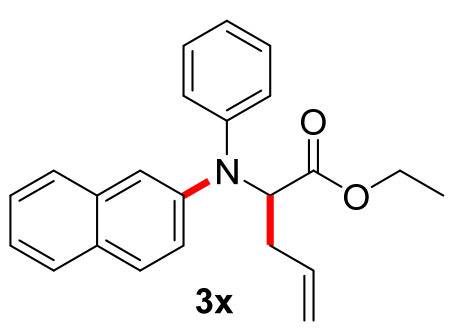

Following the general procedure, treatment of ethyl $\mathrm{N}$-allyl- $\mathrm{N}$ phenylglycinate 1a $(0.110 \mathrm{~g}, \quad 0.5 \mathrm{mmol})$ and $3-$ (trimethylsilyl)naphthalen-2-yl trifluoromethanesulfonate $2 \mathrm{e}(0.261$ $\mathrm{g}, 0.75 \mathrm{mmol})$ in the presence of $\mathrm{KF}(0.087 \mathrm{~g}, 1.5 \mathrm{mmol})$ and 18 crown-6 (0.396 g, $1.5 \mathrm{mmol})$ in THF $(2.0 \mathrm{~mL})$ at $\mathrm{rt}$ for $12 \mathrm{~h}$ followed by flash column chromatography $($ Pet. ether $/ \mathrm{EtOAc}=99 / 01)$ of the crude reaction mixture using silica gel afforded ethyl 2-(naphthalen-2-yl(phenyl)amino)pent-4-enoate $\mathbf{3 x}$ as yellow oil $\quad(0.124 \quad \mathrm{~g}, \quad 72 \% \quad$ yield). When $\quad$ 1-(trimethylsilyl)naphthalen-2-yl trifluoromethanesulfonate aryne precursor was used $\mathbf{3 x}$ was isolated in $69 \%$ yield.

$\boldsymbol{R}_{\mathbf{f}}($ Pet. ether $/$ EtOAc $=95 / 05): 0.57 ;{ }^{1} \mathbf{H}$ NMR $\left(400 \mathrm{MHz}, \mathbf{C D C l}_{3}\right) \delta 7.77(\mathrm{~d}, J=8.0 \mathrm{~Hz}, 1 \mathrm{H})$, $7.71(\mathrm{~d}, J=8.6 \mathrm{~Hz}, 2 \mathrm{H}), 7.56-7.21(\mathrm{~m}, 5 \mathrm{H}), 7.15(\mathrm{dd}, J=8.8,1.5 \mathrm{~Hz}, 1 \mathrm{H}), 7.07$ (t, $J=7.4 \mathrm{~Hz}$, $3 \mathrm{H}), 5.91-5.82(\mathrm{~m}, 1 \mathrm{H}), 5.11-5.06(\mathrm{~m}, 2 \mathrm{H}), 4.77(\mathrm{t}, J=7.5 \mathrm{~Hz}, 1 \mathrm{H}), 4.38-4.08(\mathrm{~m}, 2 \mathrm{H}), 2.74(\mathrm{t}$, $J=7.1 \mathrm{~Hz}, 2 \mathrm{H}), 1.26(\mathrm{t}, J=7.1 \mathrm{~Hz}, 3 \mathrm{H}) .{ }^{13} \mathbf{C} \mathbf{~ N M R}\left(\mathbf{1 0 0} \mathbf{M H z}, \mathbf{C D C l}_{3}\right) \delta 172.85,146.75$, 144.39, 134.59, 134.48, 129.74, 129.30, 128.83, 127.61, 127.18, 126.31, 124.32, 123.65, 123.32, 122.78, 117.88, 117.85, 63.23, 61.26, 34.39, 14.33. HRMS (ESI) calculated $[\mathrm{M}+\mathrm{H}]^{+}$for $\mathrm{C}_{23} \mathrm{H}_{24} \mathrm{O}_{2} \mathrm{~N}: 346.1802$, found: 346.1795. FTIR (cm $\left.{ }^{-1}\right)$ 3020, 2948, 2814, 1724, 1594, 1506, 1447, 1357, 1282, 1222, 1128, 1038, 956.

Ethyl-2-(phenyl(m-tolyl)amino)pent-4-enoate (3y) and Ethyl-2-(phenyl(p-tolyl)amino)pent4-enoate (3y')

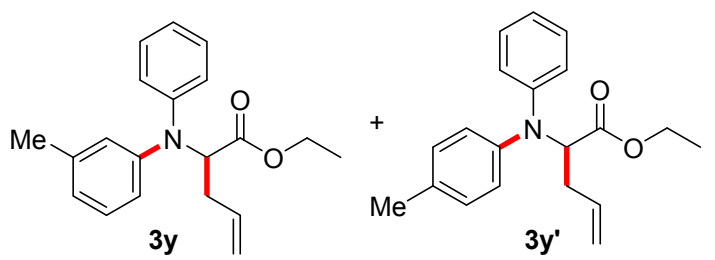

Following the general procedure, treatment of ethyl $N$-allyl- $N$-phenylglycinate 1a $(0.110 \mathrm{~g}, 0.5 \mathrm{mmol})$ and 4-methyl-2-(trimethylsilyl) phenyl trifluoromethanesulfonate $2 \mathrm{~g}(0.234 \mathrm{~g}, 182 \mu \mathrm{L}, 0.75$ $\mathrm{mmol})$ in the presence of $\mathrm{KF}(0.087 \mathrm{~g}, 1.5 \mathrm{mmol})$ and 18 -crown-6 $(0.396 \mathrm{~g}, 1.5 \mathrm{mmol})$ in THF $(2.0 \mathrm{~mL})$ at $30{ }^{\circ} \mathrm{C}$ for $12 \mathrm{~h}$ followed by flash column chromatography (Pet. ether /EtOAc $=$ 99/01) of the crude reaction mixture using silica gel afforded inseparable regioisomeric mixture of ethyl-2-(phenyl(m-tolyl)amino)pent-4-enoate $\mathbf{3 y}$ and ethyl-2-(phenyl(p-tolyl)amino)pent-4enoate $3 \mathbf{y}^{\prime}$ in $1: 1$ as yellow oil ( $0.124 \mathrm{~g}, 80 \%$ yield). 
$\boldsymbol{R}_{\mathbf{f}}($ Pet. ether $/$ EtOAc $=95 / 05): 0.57 ;{ }^{1} \mathbf{H}$ NMR (400 MHz, $\left.\mathbf{C D C l}_{3}\right) \delta$ 7.33-7.17 (m, 4H), 7.09 7.07 (m, 1H), 7.03-7.01 (d, $J=8.2 \mathrm{~Hz}, 1 \mathrm{H}), 6.93-6.85(\mathrm{~m}, 3 \mathrm{H}), 5.90-5.83(\mathrm{~m}, 1 \mathrm{H}), 5.13-5.09$ (m, 2H), 4.70-4.66 (m, 1H), 4.25-4.22 (m, 2H), 2.69-2.67 (m, 2H), 2.39 (s, 3H), 1.31 (t, J=6.7 Hz, 3H). ${ }^{13}$ C NMR (100 MHz, $\left.\mathbf{C D C l}_{3}\right) \delta 172.83,147.68,146.51,138.94,133.53,129.10,125.68$, 123.39. 121.94, 120.15, 117.63, 62.66, 61.05, 20.88 Representative peak for other isomer: ${ }^{1} \mathbf{H}$ NMR (400 MHz, $\left.\mathbf{C D C l}_{3}\right) 2.34$ (s). ${ }^{13} \mathbf{C}$ NMR (100 MHz, $\left.\mathbf{C D C l}_{3}\right) \delta$ 172.80, 146.75, 143.16, $134.61,129.92,129.0,123.73,122.23,120.40,119.42,62.58,21.62,14.25$. HRMS (ESI) calculated $[\mathrm{M}+\mathrm{H}]^{+}$for $\mathrm{C}_{20} \mathrm{H}_{24} \mathrm{O}_{2} \mathrm{~N}: 310.1802$, found: 310.1797 . FTIR (cm $\left.{ }^{-1}\right)$ 2982, 2929, 1733, $1641,1498,1217,1129,1030,993,772,703$.

\section{Ethyl-2-((4-fluorophenyl)(phenyl)amino)pent-4-enoate (3z) and Ethyl-2-((3 fluorophenyl)} (phenyl)amino)pent-4-enoate $\left(3 z^{\prime}\right)$

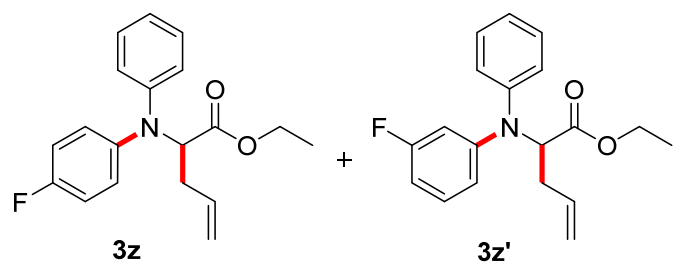

Following the general procedure, treatment of ethyl $N$ allyl- $N$-phenylglycinate $1 \mathrm{a}(0.110 \mathrm{~g}, 0.5 \mathrm{mmol})$ and 4fluoro-2-(trimethylsilyl)phenyl trifluoromethane sulfonate $2 \mathbf{h}(0.237 \mathrm{~g}, 0.75 \mathrm{mmol})$ in the presence of KF (0.087 g, $1.5 \mathrm{mmol})$ and 18-crown-6 (0.396 g, 1.5 $\mathrm{mmol})$ in $\mathrm{THF}(2.0 \mathrm{~mL})$ at $30^{\circ} \mathrm{C}$ for $12 \mathrm{~h}$ followed by flash column chromatography (Pet. ether $/$ EtOAc $=99 / 01) \quad$ of the crude reaction mixture using silica gel afforded inseparable 2:1 regioisomeric mixture of ethyl-2-((4-fluorophenyl)(phenyl)amino)pent-4-enoate $\mathbf{3 z}$ and ethyl-2((3 fluorophenyl)(phenyl)amino)pent-4-enoate 3z' as yellow oil (0.123 g, 78\% yield).

$\boldsymbol{R}_{\mathbf{f}}($ Pet. ether $/$ EtOAc $=95 / 05): 0.52 ;{ }^{1} \mathbf{H}$ NMR (400 MHz, $\left.\mathbf{C D C l}_{3}\right) \delta 7.24-7.08(\mathrm{~m}, 4 \mathrm{H}), 7.02$ $(\mathrm{t}, J=8.0,2 \mathrm{H}), 6.88(\mathrm{t}, J=7.3 \mathrm{~Hz}, 1 \mathrm{H}), 6.77(\mathrm{~d}, J=8.1 \mathrm{~Hz}, 2 \mathrm{H}), 5.95-5.62(\mathrm{~m}, 1 \mathrm{H}), 5.08-5.05$ $(\mathrm{m}, 2 \mathrm{H}), 4.60(\mathrm{t}, J=7.5 \mathrm{~Hz}, 1 \mathrm{H}), 4.29-4.06(\mathrm{~m}, 2 \mathrm{H}), 2.79-2.39(\mathrm{~m}, 2 \mathrm{H}), 1.24(\mathrm{t}, J=7.1 \mathrm{~Hz}$, 3H). ${ }^{13}$ C NMR (100 MHz, $\left.\mathbf{C D C l}_{3}\right) \delta 172.81,159.92$ (d, $J=242.7 \mathrm{~Hz}$ ), 147.78, 134.41, 129.19, $128.28(\mathrm{~d}, J=242.7 \mathrm{~Hz}), 127.06,118.74,117.90,116.20,62.61,61.24,34.64,14.34 .{ }^{1} \mathbf{H}$ NMR $\left(400 \mathrm{MHz}, \mathbf{C D C l}_{3}\right) \delta 7.24-7.08(\mathrm{~m}, 4 \mathrm{H}), 7.02(\mathrm{t}, J=8.0,2 \mathrm{H}), 6.88(\mathrm{t}, J=7.3 \mathrm{~Hz}, 1 \mathrm{H}), 6.77$ (d, $J=8.1 \mathrm{~Hz}, 2 \mathrm{H}), 5.95-5.62(\mathrm{~m}, 1 \mathrm{H}), 5.08-5.05(\mathrm{~m}, 2 \mathrm{H}), 4.60(\mathrm{t}, J=7.5 \mathrm{~Hz}, 1 \mathrm{H}), 4.29-4.06(\mathrm{~m}$, 2H), $2.79-2.39(\mathrm{~m}, 2 \mathrm{H}), 1.24(\mathrm{t}, J=7.1 \mathrm{~Hz}, 3 \mathrm{H}) .{ }^{13} \mathbf{C}$ NMR (100 MHz, CDCl $\left.\mathbf{3}\right) \delta 172.81$, $159.92(\mathrm{~d}, J=242.7 \mathrm{~Hz}), 141.56,129.58,125.21,120.46,118.00,116.20,115.98,62.77,61.35$, 
34.51, 14.34. HRMS (ESI) calculated $[\mathrm{M}+\mathrm{H}]^{+}$for $\mathrm{C}_{19} \mathrm{H}_{21} \mathrm{O}_{2} \mathrm{NF}: 314.1551$, found: 314.1549 . FTIR (cm $\left.{ }^{-1}\right)$ 3019, 2983, 2402, 1733, 1594, 1504, 1370, 1217, 1030, 923, 771, 669.

\section{Ethyl 2-(diphenylamino)-3-phenylpent-4-enoate (4)}<smiles>C=CC(c1ccccc1)C(C(=O)OCC)N(c1ccccc1)c1ccccc1</smiles>

Following the general procedure, treatment of ethyl $N$-cinnamyl- $N$ phenylglycinate $(0.198 \mathrm{~g}, 0.5 \mathrm{mmol})$ and 2-(trimethylsilyl)phenyl trifluoromethane sulfonate $\mathbf{2 a}(0.223 \mathrm{~g}, 182 \mu \mathrm{L}, 0.75 \mathrm{mmol})$ in the presence of $\mathrm{KF}(0.087 \mathrm{~g}, 1.5 \mathrm{mmol})$ and 18-crown-6 (0.396 g, $1.5 \mathrm{mmol})$ in THF

$(2.0 \mathrm{~mL})$ at $\mathrm{rt}$ for $12 \mathrm{~h}$ followed by flash column chromatography (Pet. ether /EtOAc $=99 / 01)$ of the crude reaction mixture using silica gel afforded ethyl 2-(diphenylamino)-3-phenylpent-4enoate 4 as yellow oil $(0.137 \mathrm{~g}, 74 \%$ yield $)$.

$\boldsymbol{R}_{\mathbf{f}}($ Pet. ether $/$ EtOAc $=95 / 05): 0.51 ;{ }^{1} \mathbf{H}$ NMR (400 MHz, $\left.\mathbf{C D C l}_{3}\right) \delta 7.37-7.27(\mathrm{~m}, 9 \mathrm{H}), 7.21$ $(\mathrm{d}, J=7.6 \mathrm{~Hz}, 4 \mathrm{H}), 7.10(\mathrm{t}, J=7.2 \mathrm{~Hz}, 2 \mathrm{H}), 6.21-6.12(\mathrm{~m}, 1 \mathrm{H}), 5.14(\mathrm{t}, J=11.9 \mathrm{~Hz}, 2 \mathrm{H}), 5.01$ $(\mathrm{d}, J=17.3 \mathrm{~Hz}, 1 \mathrm{H}), 4.14\left(\mathrm{dd}, J_{1}=7.2 \mathrm{~Hz}, J_{2}=10.7 \mathrm{~Hz}, 1 \mathrm{H}\right), 4.00-3.72(\mathrm{~m}, 2 \mathrm{H}), 0.95(\mathrm{t}, J=$ $7.1 \mathrm{~Hz}, 3 \mathrm{H}) .{ }^{13} \mathbf{C}$ NMR (100 MHz, $\left.\mathbf{C D C l}_{3}\right) \delta 170.97,147.12,140.60,138.57,129.18,128.93$, 128.53, 127.04, 123.19, 122.57, 116.96, 66.81, 60.67, 50.09, 13.82. HRMS (ESI) calculated $[\mathrm{M}+\mathrm{H}]^{+}$for $\mathrm{C}_{25} \mathrm{H}_{26} \mathrm{O}_{2} \mathrm{~N}: 372.1958$, found: 372.1959. FTIR (cm $\left.\mathbf{c m}^{-\mathbf{1}}\right)$ 3020, 2953, 1722, 1590, $1485,1443,1348,1292,1253,1216,1080,893,760$.

\section{Ethyl-2-(diphenylamino)-3-methylpent-4-enoate (5)}

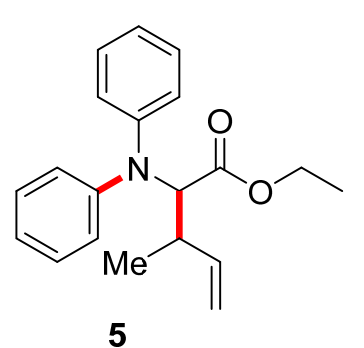

Following the general procedure, treatment of ethyl (E)- $N$-(but-2-en-1-yl)$N$-phenylglycinate $(0.117 \mathrm{~g}, 0.5 \mathrm{mmol})$ and 2-(trimethylsilyl)phenyl trifluoromethane sulfonate $\mathbf{2 a}(0.223 \mathrm{~g}, 182 \mu \mathrm{L}, 0.75 \mathrm{mmol})$ in the presence of $\mathrm{KF}(0.087 \mathrm{~g}, 1.5 \mathrm{mmol})$ and 18-crown-6 (0.396 g, 1.5 $\mathrm{mmol})$ in THF $(2.0 \mathrm{~mL})$ at $30{ }^{\circ} \mathrm{C}$ for $12 \mathrm{~h}$ followed by flash column chromatography (Pet. ether $/$ EtOAc $=99 / 01)$ of the crude reaction mixture using silica gel afforded Ethyl-2-(diphenylamino)-3-methylpent-4-enoate 5 as yellow oil (0.125 g, 81\% yield).

$\boldsymbol{R}_{\mathbf{f}}($ Pet. ether $/$ EtOAc $=95 / 05): 0.60 ;{ }^{1} \mathbf{H}$ NMR (400 MHz, $\left.\mathbf{C D C l}_{3}\right) \delta$ 7.31-7.26 (m, 4H), 7.12$7.07(\mathrm{~m}, 3 \mathrm{H}), 7.02(\mathrm{t}, J=7.4 \mathrm{~Hz}, 2 \mathrm{H}), 5.90-5.84(\mathrm{~m}, 1 \mathrm{H}), 5.03-4.95(\mathrm{~m}, 2 \mathrm{H}), 4.46(\mathrm{~d}, J=10.2$ $\mathrm{Hz}, 1 \mathrm{H}), 4.23-4.15(\mathrm{~m}, 2 \mathrm{H}), 3.05-3.00(\mathrm{~m}, 1 \mathrm{H}), 1.21(\mathrm{t}, J=7.1 \mathrm{~Hz}, 3 \mathrm{H}), 1.12(\mathrm{~d}, J=6.7 \mathrm{~Hz}, 3 \mathrm{H})$. 
${ }^{13}$ C NMR (100 MHz, $\left.\mathbf{C D C l}_{3}\right) \delta 171.96,147.41,140.76,129.23,129.09,123.04,122.32,115.13$, 68.13, 60.92, 37.80, 18.30, 14.29. HRMS (ESI) calculated $[\mathrm{M}+\mathrm{H}]^{+}$for $\mathrm{C}_{20} \mathrm{H}_{24} \mathrm{O}_{2} \mathrm{~N}: 310.1802$, found: 310.1797. FTIR (cm $\left.{ }^{-1}\right)$ 3064, 3018, 2981, 2876, 1732, 1590, 1419, 1352, 1098, 993, 701, 668.

Methyl (S)-2-allyl-1-phenylpyrrolidine-2-carboxylate (7a)

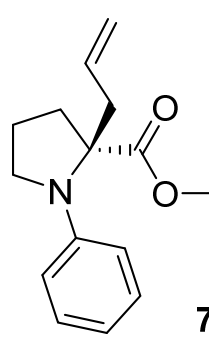

Following the general procedure, treatment of methyl allyl- $L$-prolinate $\mathbf{6 a}$ $(0.085 \mathrm{~g}, 0.5 \mathrm{mmol})$ and 2-(trimethylsilyl)phenyl trifluoromethane sulfonate $\mathbf{2 a}$ $(0.223 \mathrm{~g}, 182 \mu \mathrm{L}, 0.75 \mathrm{mmol})$ in the presence of $\mathrm{KF}(0.087 \mathrm{~g}, 1.5 \mathrm{mmol})$ and 18-crown-6 $(0.396 \mathrm{~g}, 1.5 \mathrm{mmol})$ in THF $(2.0 \mathrm{~mL})$ at $\mathrm{rt}$ for $2 \mathrm{~h}$ followed by flash 7a column chromatography (Pet. ether $/$ EtOAc $=99 / 01)$ of the crude reaction mixture using silica gel afforded methyl (S)-2-allyl-1-phenylpyrrolidine-2-carboxylate 7a as yellow oil $(0.077 \mathrm{~g}, 63 \%$ yield $)$.

$\boldsymbol{R}_{\mathbf{f}}($ Pet. ether $/$ EtOAc $=95 / 05): 0.52 ; 96 \%$ ee, $[\alpha]_{\mathrm{D}}{ }^{25}=+41.22\left(\right.$ c $\left.0.1, \mathrm{CHCl}_{3}\right)$. HPLC $($ Chiralcel OD-H, 98 : 2 (hexane:IPA), $0.8 \mathrm{~mL} / \mathrm{min}$ ) Major: $6.9 \mathrm{~min}$, Minor: $6.1 \mathrm{~min} .{ }^{1} \mathbf{H}$ NMR (400 MHz, $\left.\mathbf{C D C l}_{3}\right) \delta 7.20(\mathrm{t}, J=7.8 \mathrm{~Hz}, 2 \mathrm{H}), 6.70(\mathrm{t}, J=7.2 \mathrm{~Hz}, 1 \mathrm{H}), 6.55(\mathrm{~d}, J=8.2 \mathrm{~Hz}, 2 \mathrm{H}), 5.63-5.52$ (m 1H), $5.06(\mathrm{~d}, J=4.8 \mathrm{~Hz}, 1 \mathrm{H}), 5.03(\mathrm{~s}, 1 \mathrm{H}), 3.69$ (s, 3H), $3.51(\mathrm{dd}, J=9.5,4.1 \mathrm{~Hz}, 2 \mathrm{H}), 3.11$ $(\mathrm{dd}, J=14.5,5.6 \mathrm{~Hz}, 1 \mathrm{H}), 2.71(\mathrm{dd}, J=14.5,8.7 \mathrm{~Hz}, 1 \mathrm{H}), 2.35-1.88(\mathrm{~m}, 4 \mathrm{H}) .{ }^{13} \mathbf{C}$ NMR (100 MHz, $\left.\mathbf{C D C l}_{3}\right) \delta 177.13,145.72,133.49,129.11,118.91,116.64,113.22,67.83,52.44,50.95$, 37.87, 37.71, 23.00. HRMS (ESI) calculated $[\mathrm{M}+\mathrm{H}]^{+}$for $\mathrm{C}_{15} \mathrm{H}_{20} \mathrm{O}_{2} \mathrm{~N}$ : 246.1489, found: 246.1485. FTIR (cm $\left.{ }^{-1}\right)$ 3016, 2925, 1691, 1640, 1587, 1494, 1396, 1217, 1179, 1032, 918, 771.

\section{Ethyl (S)-2-allyl-1-phenylpyrrolidine-2-carboxylate (7b)}

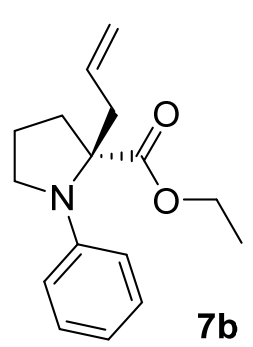

Following the general procedure, treatment of ethyl allyl- $L$-prolinate $6 \mathbf{b}(0.92 \mathrm{~g}$, $0.5 \mathrm{mmol})$ and 2-(trimethylsilyl)phenyl trifluoromethane sulfonate $\mathbf{2 a}(0.223 \mathrm{~g}$, $182 \mu \mathrm{L}, 0.75 \mathrm{mmol})$ in the presence of $\mathrm{KF}(0.087 \mathrm{~g}, 1.5 \mathrm{mmol})$ and 18-crown$6(0.396 \mathrm{~g}, 1.5 \mathrm{mmol})$ in THF $(2.0 \mathrm{~mL})$ at $\mathrm{rt}$ for $2 \mathrm{~h}$ followed by flash column 7b chromatography (Pet. ether $/$ EtOAc $=99 / 01$ ) of the crude reaction mixture using silica gel afforded ethyl (S)-2-allyl-1-phenylpyrrolidine-2-carboxylate 7a as yellow oil (0.079 g, 61\% yield). 
$\boldsymbol{R}_{\mathbf{f}}($ Pet. ether $/$ EtOAc $=95 / 05): 0.50 ; 98 \%$ ee; $[\alpha]_{\mathrm{D}}{ }^{25}=+57.16\left(\mathrm{c} 0.1, \mathrm{CHCl}_{3}\right)$. HPLC $($ Chiralpak IB, 99 : 1 (hexane:IPA), $0.7 \mathrm{~mL} / \mathrm{min}$ ) Major: $6.5 \mathrm{~min}$, Minor: 5.9 min. ${ }^{1} \mathbf{H}$ NMR (400 MHz, $\left.\mathbf{C D C l}_{3}\right) \delta 7.20(\mathrm{t}, J=7.8 \mathrm{~Hz}, 2 \mathrm{H}), 6.70(\mathrm{t}, J=7.2 \mathrm{~Hz}, 1 \mathrm{H}), 6.58(\mathrm{~d}, J=8.2 \mathrm{~Hz}, 2 \mathrm{H}), 5.66-5.56$ (m 1H), $5.07(\mathrm{~d}, J=4.8 \mathrm{~Hz}, 1 \mathrm{H}), 5.04(\mathrm{~s}, 1 \mathrm{H}), 4.24-4.10(\mathrm{~m}, 2 \mathrm{H}), 3.55-3.47(\mathrm{~m}, 2 \mathrm{H}), 3.13$ (dd, $J=9.5,4.1 \mathrm{~Hz}, 1 \mathrm{H}), 2.72(\mathrm{dd}, J=14.5,5.6 \mathrm{~Hz}, 1 \mathrm{H}), 2.32-1.94(\mathrm{~m}, 4 \mathrm{H}), 1.17$ (t, $J=8.2$ $\mathrm{Hz}, 3 \mathrm{H}) .{ }^{13} \mathbf{C}$ NMR (100 MHz, $\left.\mathbf{C D C l}_{3}\right) \delta 176.44,145.85,133.64,128.98,118.74,116.57$, 113.29, 67.93, 61.13, 51.00, 37.91, 37.83, 23.01, 14.19. HRMS (ESI) calculated $[\mathrm{M}+\mathrm{H}]^{+}$for $\mathrm{C}_{16} \mathrm{H}_{22} \mathrm{O}_{2} \mathrm{~N}: 260.1645$, found: 260.1643. FTIR $\left(\mathbf{c m}^{-1}\right)$ 3017, 2982, 1731, 1589, 1445, 1417, 1374, 1352, 1292, 1120, 1029, 922, 773.

\section{Methyl (S)-2-allyl-1-(benzo[d][1,3] dioxol-5-yl)pyrrolidine-2-carboxylate (7c)}

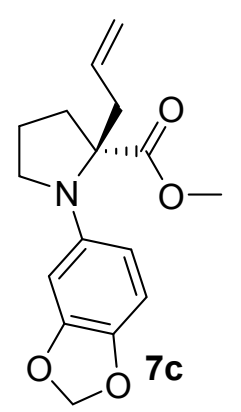

Following the general procedure, treatment of ethyl allyl- $L$-prolinate $6 \mathbf{a}(0.085 \mathrm{~g}$, $0.5 \mathrm{mmol}$ ) and 6-(trimethylsilyl)benzo[ $d][1,3]$ dioxol-5-yl trifluoromethanesulfonate $2 \mathbf{c}(0.257 \mathrm{~g}, 0.75 \mathrm{mmol})$ in the presence of $\mathrm{KF}(0.087$ $\mathrm{g}, 1.5 \mathrm{mmol})$ and 18 -crown-6 $(0.396 \mathrm{~g}, 1.5 \mathrm{mmol})$ in THF $(2.0 \mathrm{~mL})$ at $\mathrm{rt}$ for $2 \mathrm{~h}$ followed by flash column chromatography (Pet. ether $/$ EtOAc $=99 / 01$ ) of the crude reaction mixture using silica gel afforded methyl (S)-2-allyl-1(benzo[d][1,3]dioxol-4-yl)pyrrolidine-2-carboxylate $7 \mathbf{c}$ as yellow oil $(0.088 \mathrm{~g}, 61 \%$ yield).

$\boldsymbol{R}_{\mathbf{f}}($ Pet. ether $/$ EtOAc $=95 / 05): 0.44 ; 88 \%$ ee; $[\alpha]_{\mathrm{D}}{ }^{25}=+51.74\left(\mathrm{c} 0.1, \mathrm{CHCl}_{3}\right)$. HPLC $($ Chiralcel OD-H, 98 : 2 (HEXANE:IPA), $0.8 \mathrm{~mL} / \mathrm{min}$ ) Major: $18.1 \mathrm{~min}$, Minor: 10.8 min. ${ }^{1}$ H NMR (400 MHz, $\left.\mathbf{C D C l}_{3}\right) \delta 6.66(\mathrm{~d}, J=8.5 \mathrm{~Hz}, 1 \mathrm{H}), 6.20(\mathrm{~d}, J=2.0 \mathrm{~Hz}, 1 \mathrm{H}), 5.95(\mathrm{dd}, J=8.5,2.1 \mathrm{~Hz}, 1 \mathrm{H})$, $5.84(\mathrm{~d}, J=5.6 \mathrm{~Hz}, 2 \mathrm{H}), 5.60-5.50$ (m, 1H), 5.03 (d, $J=12.8 \mathrm{~Hz}, 2 \mathrm{H}), 3.69$ (s, 3H), 3.43 (dd, $J$ = 9.4, 4.6 Hz, 2H), $3.03(\mathrm{dd}, J=14.6,5.6 \mathrm{~Hz}, 1 \mathrm{H}), 2.65(\mathrm{dd}, J=14.6,8.7 \mathrm{~Hz}, 1 \mathrm{H}), 2.29-1.93$ (m, 4H). ${ }^{13} \mathbf{C}$ NMR (100 MHz, $\left.\mathbf{C D C l}_{3}\right) \delta 177.00,148.28,141.61,139.02,133.43,118.93$, 108.56, 105.30, 100.60, 96.33, 68.08, 52.42, 51.66, 37.80, 37.76, 22.98. HRMS (ESI) calculated $[\mathrm{M}+\mathrm{H}]^{+}$for $\mathrm{C}_{16} \mathrm{H}_{20} \mathrm{O}_{4} \mathrm{~N}: 290.1387$, found: 290.1385. FTIR $\left(\mathbf{c m}^{-1}\right)$ 3018, 2930, 2400, 1731, 1629, 1594, 1496, 1442, 1290, 1120, 994, 767. 


\section{Ethyl diphenyl-phenylalaninate (9)}

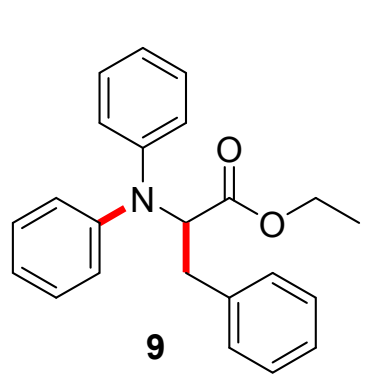

Following the general procedure, treatment of ethyl $\mathrm{N}$-benzyl- $\mathrm{N}$ phenylglycinate 8 (0.135 g, $0.5 \mathrm{mmol})$ and 2-(trimethylsilyl)phenyl trifluoromethane sulfonate $2 \mathrm{a}(0.223 \mathrm{~g}, 182 \mu \mathrm{L}, 0.75 \mathrm{mmol})$ in the presence of KF $(0.087 \mathrm{~g}, 1.5 \mathrm{mmol})$ and 18-crown-6 (0.396 g, 1.5 $\mathrm{mmol})$ in THF $(2.0 \mathrm{~mL})$ at $\mathrm{rt}$ for $12 \mathrm{~h}$ followed by flash column chromatography $($ Pet. ether $/$ EtOAc $=99 / 01)$ of the crude reaction mixture using silica gel afforded ethyl diphenyl-phenylalaninate 9 as yellow oil $(0.070 \mathrm{~g}, 41 \%$ yield).

$\boldsymbol{R}_{\mathbf{f}}\left(\right.$ Pet. ether $/$ EtOAc $=$ 95/05): 0.60; ${ }^{1} \mathbf{H}$ NMR $\left(\mathbf{4 0 0} \mathbf{~ M H z}, \mathbf{C D C l}_{3}\right) \delta 7.49-7.36(\mathrm{~m}, 7 \mathrm{H}), 7.27$ (d, $J=6.8 \mathrm{~Hz}, 2 \mathrm{H}), 7.15$ (t, $J=7.3 \mathrm{~Hz}, 2 \mathrm{H}), 6.99$ (d, $J=8.0 \mathrm{~Hz}, 4 \mathrm{H}), 4.93(\mathrm{~d}, J=8.2 \mathrm{~Hz}, 1 \mathrm{H})$, 4.48 - $4.16(\mathrm{~m}, 2 \mathrm{H}), 3.46$ - $3.44(\mathrm{~m}, 2 \mathrm{H}), 1.35(\mathrm{t}, J=7.1 \mathrm{~Hz}, 3 \mathrm{H}) .{ }^{13} \mathbf{C}$ NMR $\left(\mathbf{1 0 0} \mathbf{~ M H z}, \mathbf{C D C l}_{3}\right) \delta$ $172.89,146.96,138.40,129.36,129.17,128.66,128.49,128.36,126.67,122.56,122.18,65.52$, 61.25, 35.59, 14.18. HRMS (ESI) calculated $[\mathrm{M}+\mathrm{H}]^{+}$for $\mathrm{C}_{23} \mathrm{H}_{24} \mathrm{O}_{2} \mathrm{~N}$ : 346.1802, found: 346.1797. FTIR (cm ( $\left.^{-1}\right)$ 3022, 2951, 2256, 1722, 1598, 1517, 1445, 1362, 1288, 1082, 952, 909 , 760. 


\section{7. ${ }^{1} \mathrm{H}$ and ${ }^{13} \mathrm{C}$ NMR Spectra of Homoallylic Amines}

Ethyl-2-(diphenylamino)pent-4-enoate (3a)
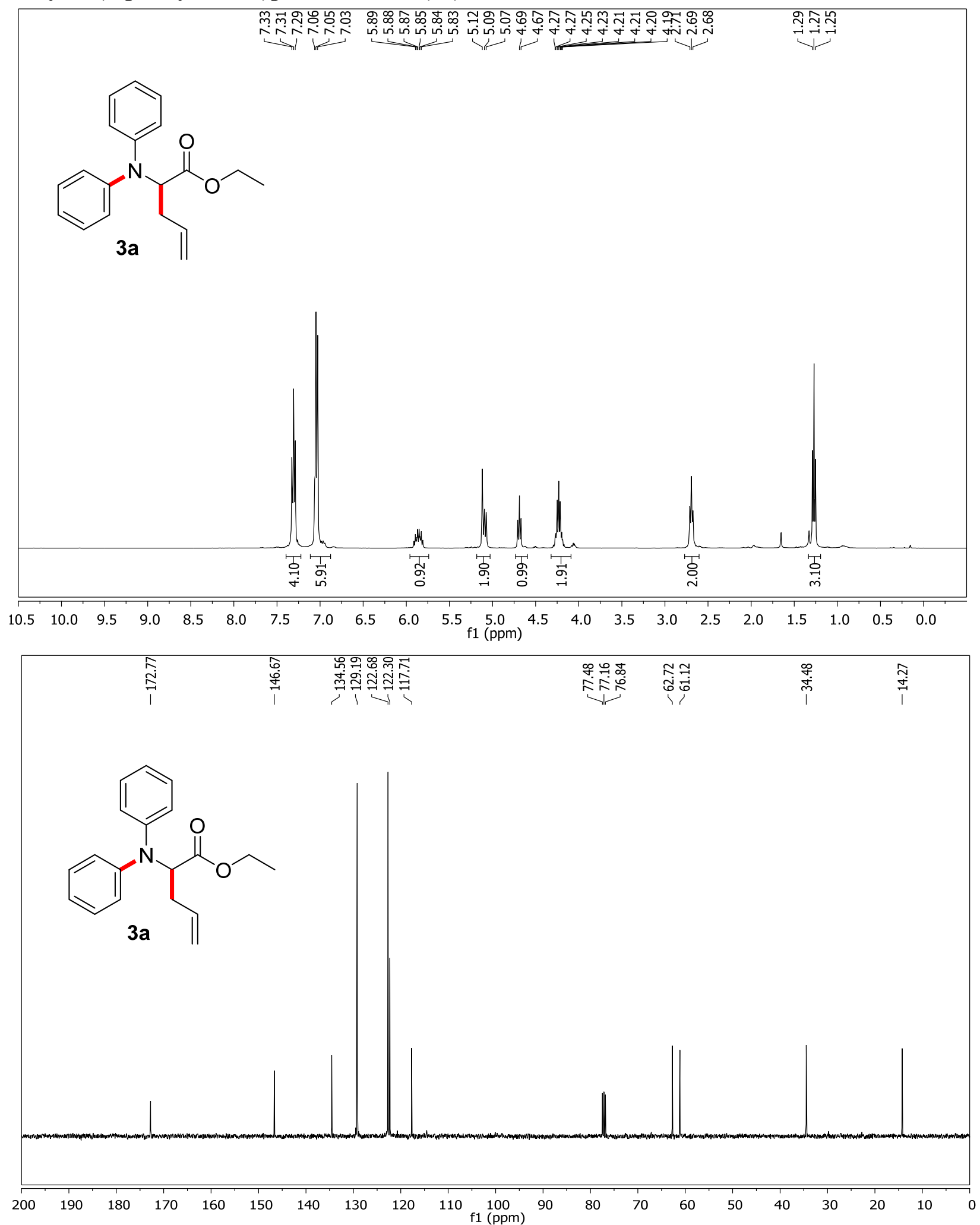
Ethyl -2-((4-methoxyphenyl)(phenyl)amino)pent-4-enoate (3b)

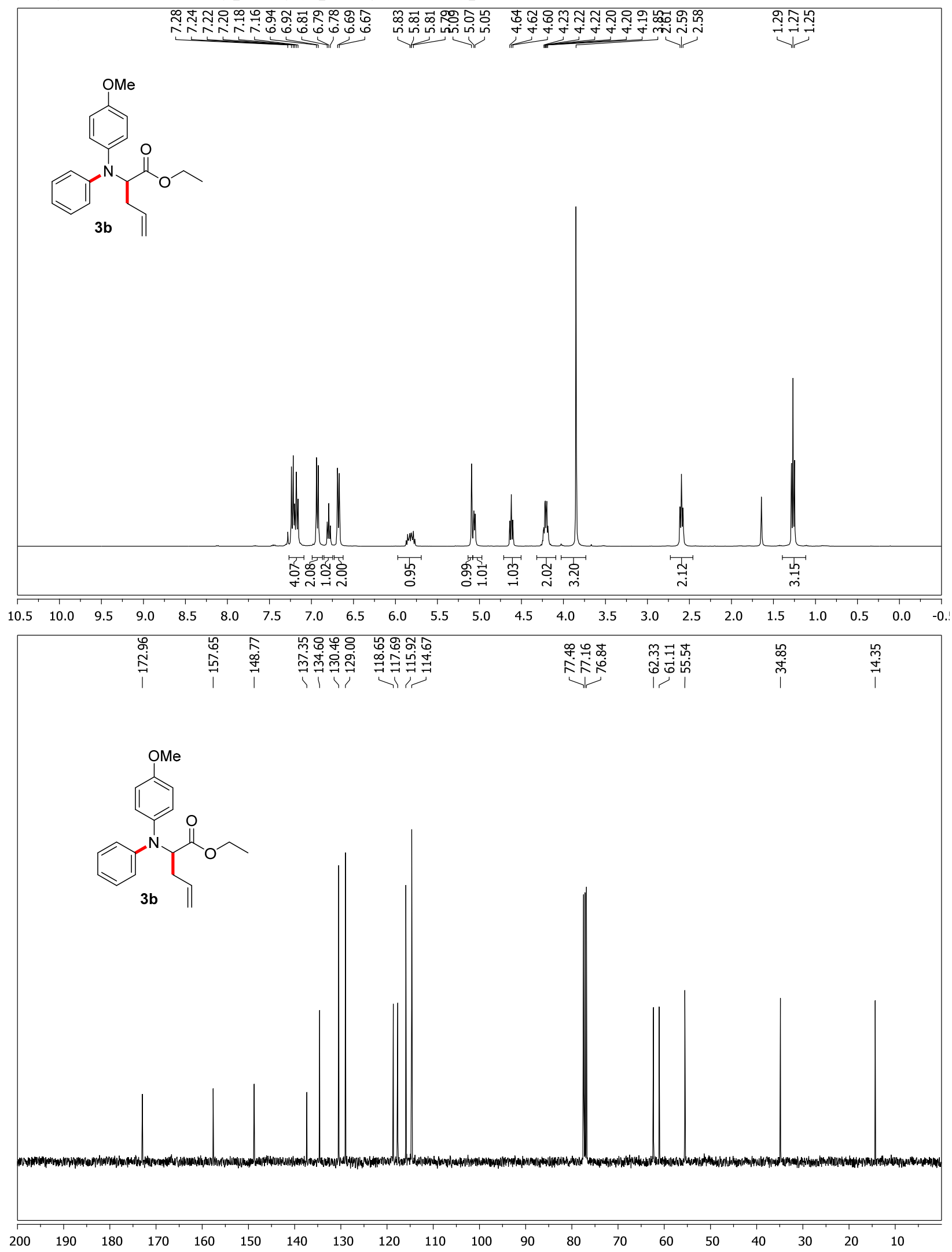


Ethyl -2-(phenyl(p-tolyl)amino)pent-4-enoate (3c)

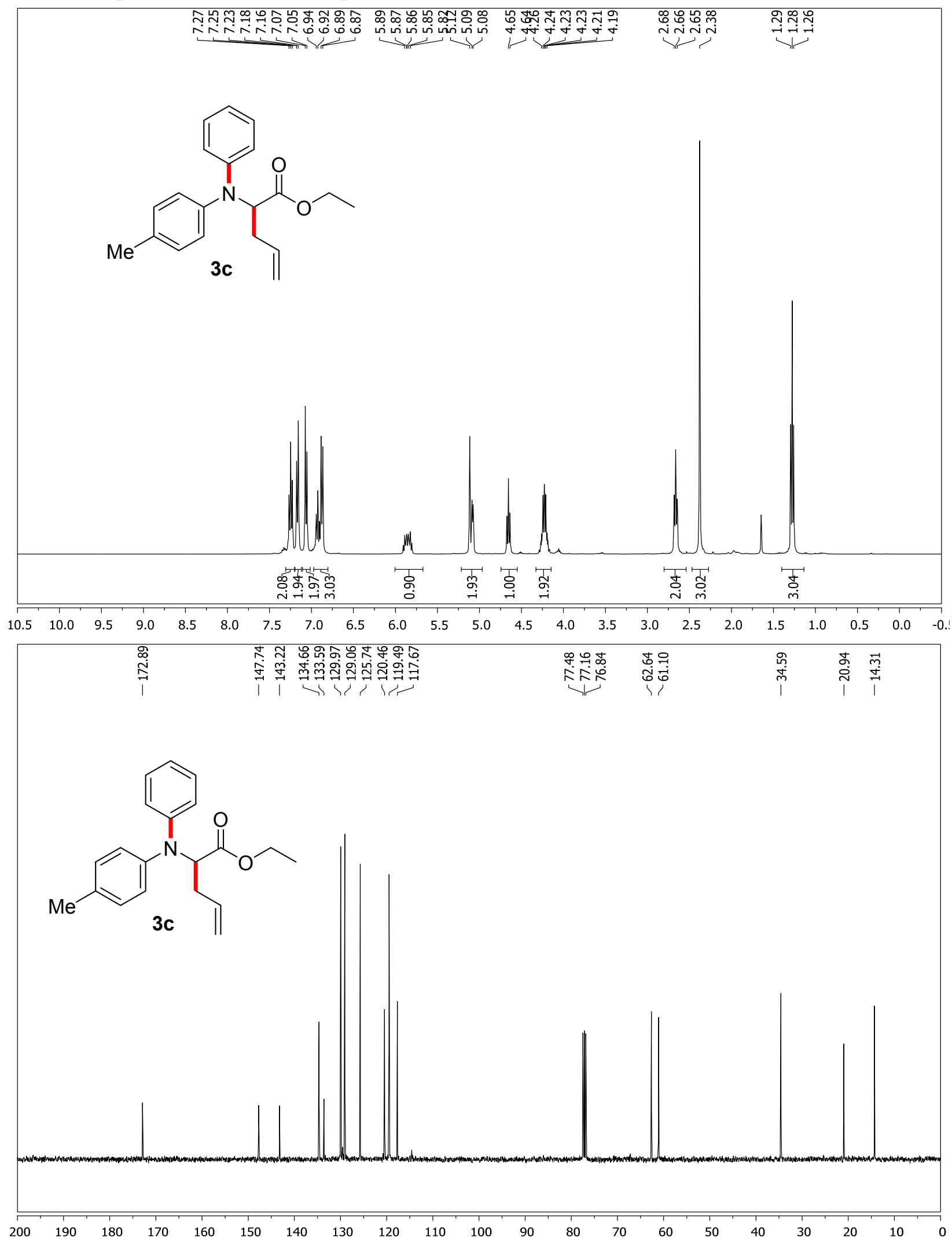


Ethyl-2-((4-bromophenyl)(phenyl)amino)pent-4-enoate (3d)

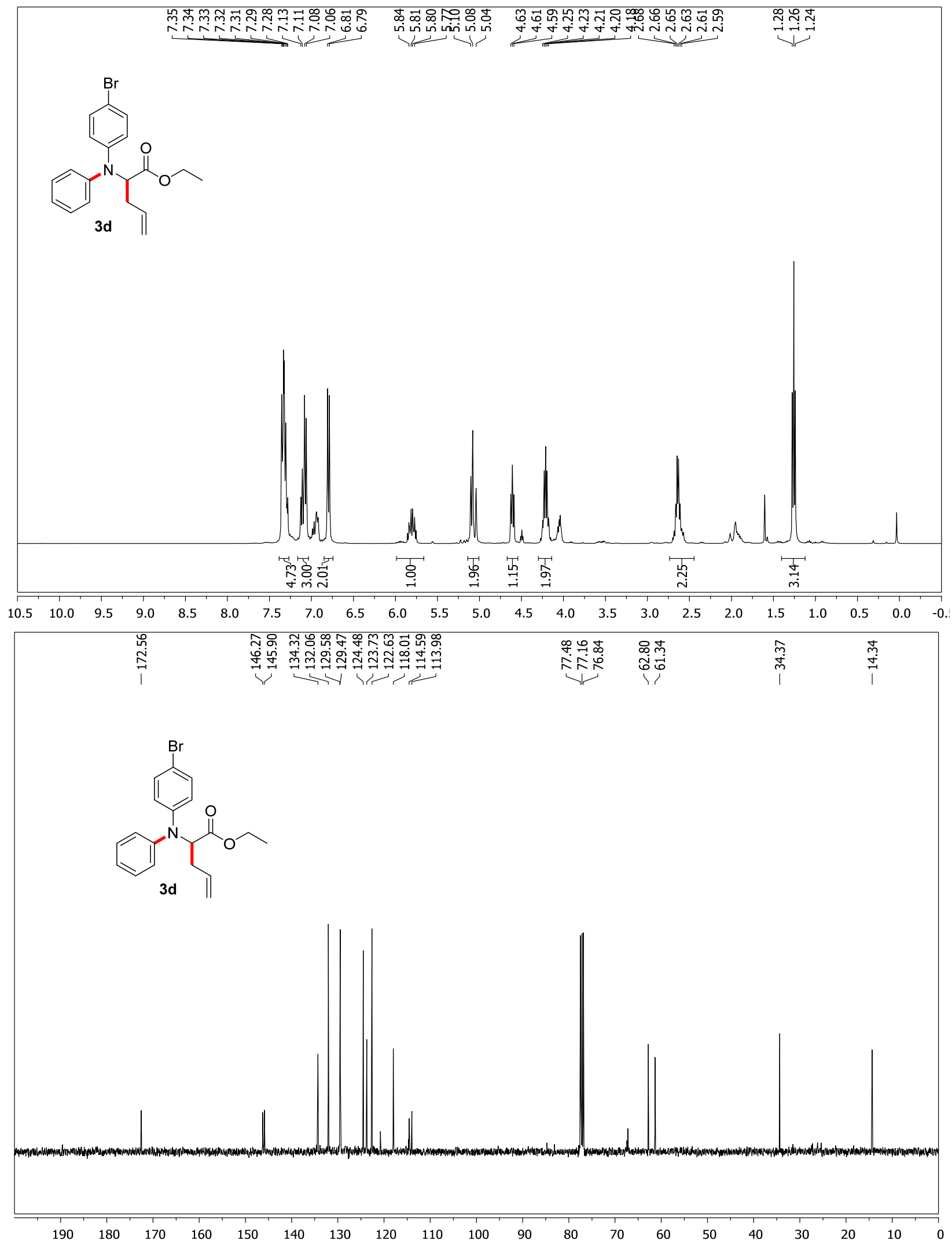


Ethyl-2-((4-chlorophenyl)(phenyl)amino)pent-4-enoate (3e)
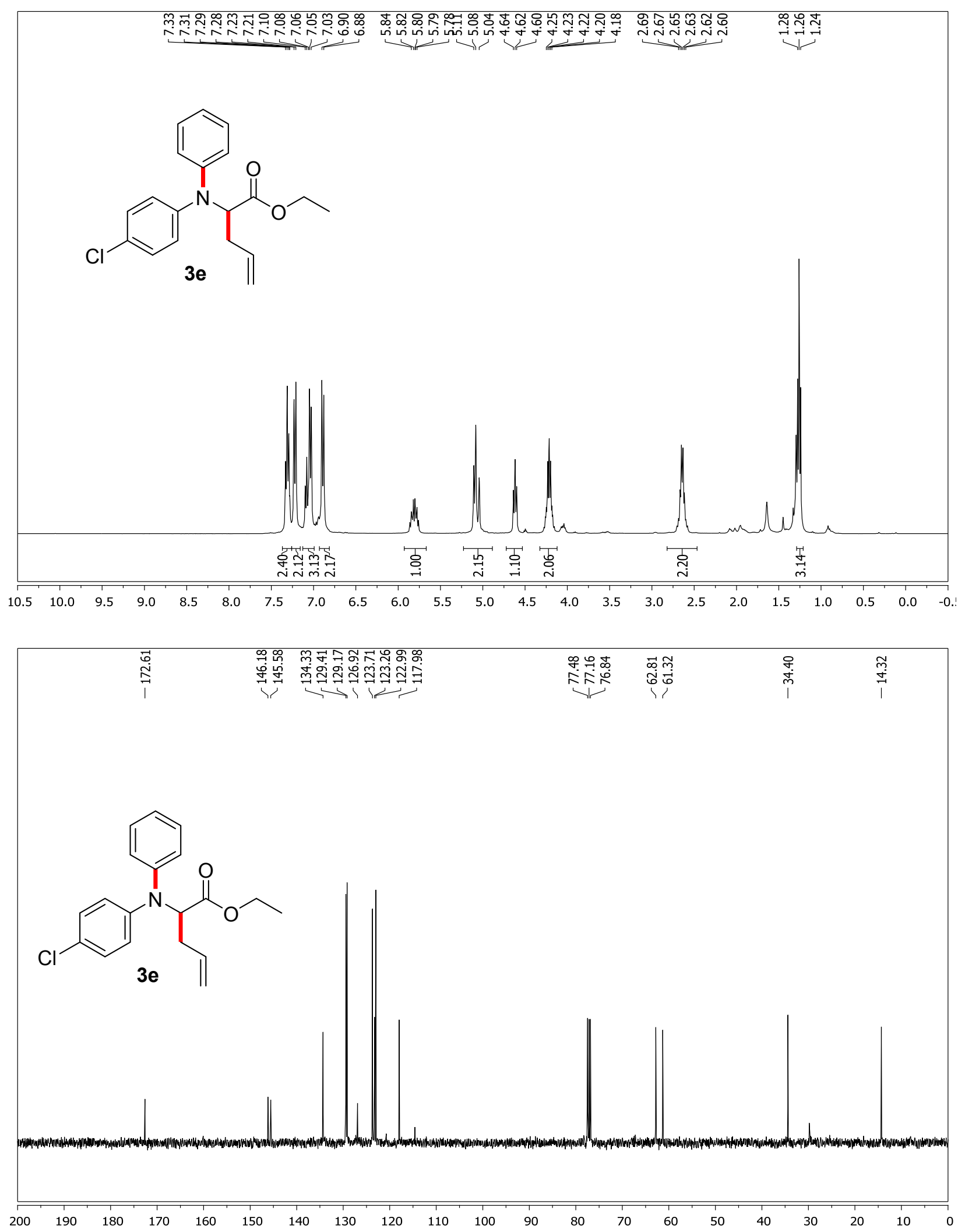
Ethyl-2-((4-fluorophenyl)(phenyl)amino)pent-4-enoate (3f)

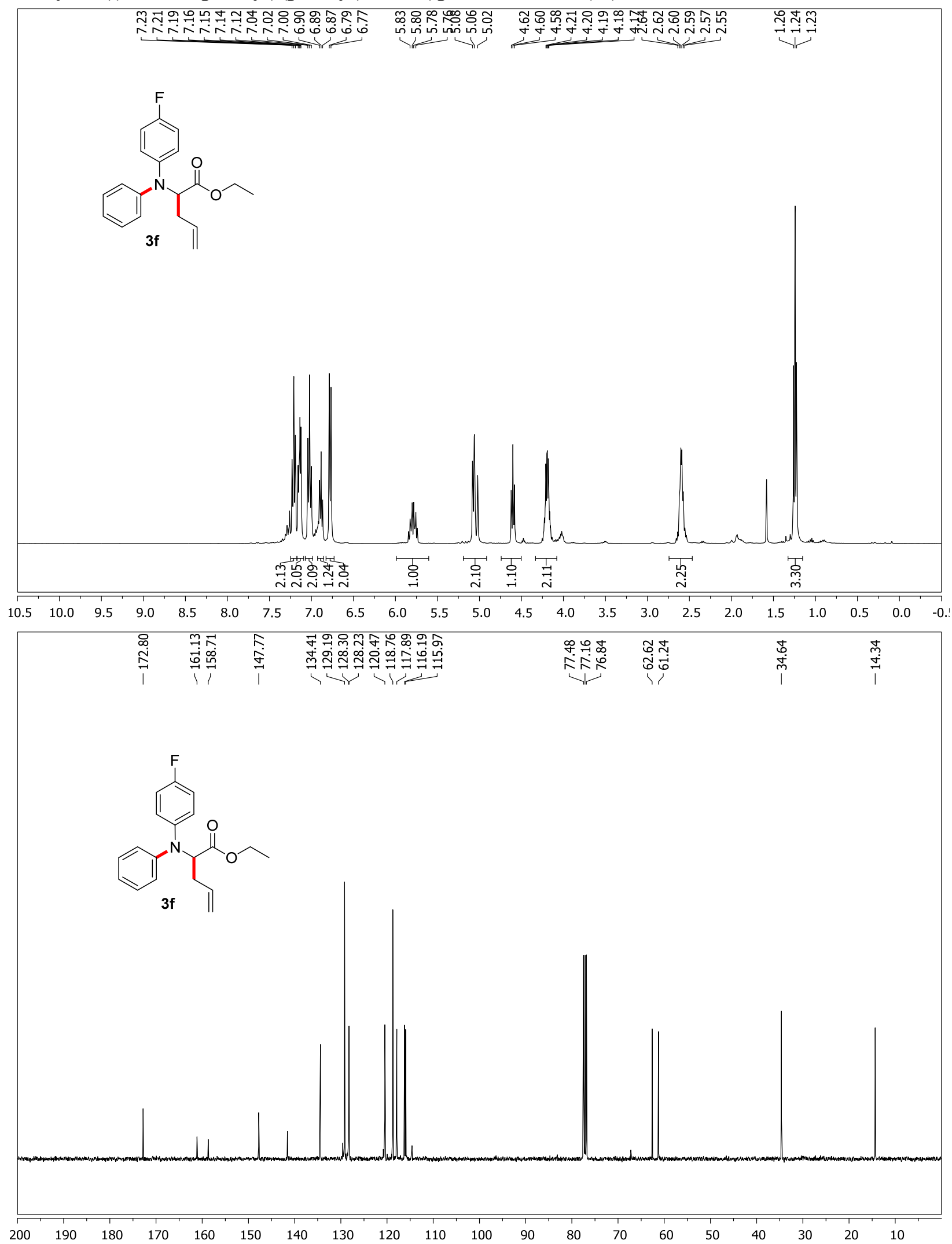


Ethyl -2-(phenyl( $m$-tolyl)amino)pent-4-enoate (3g)
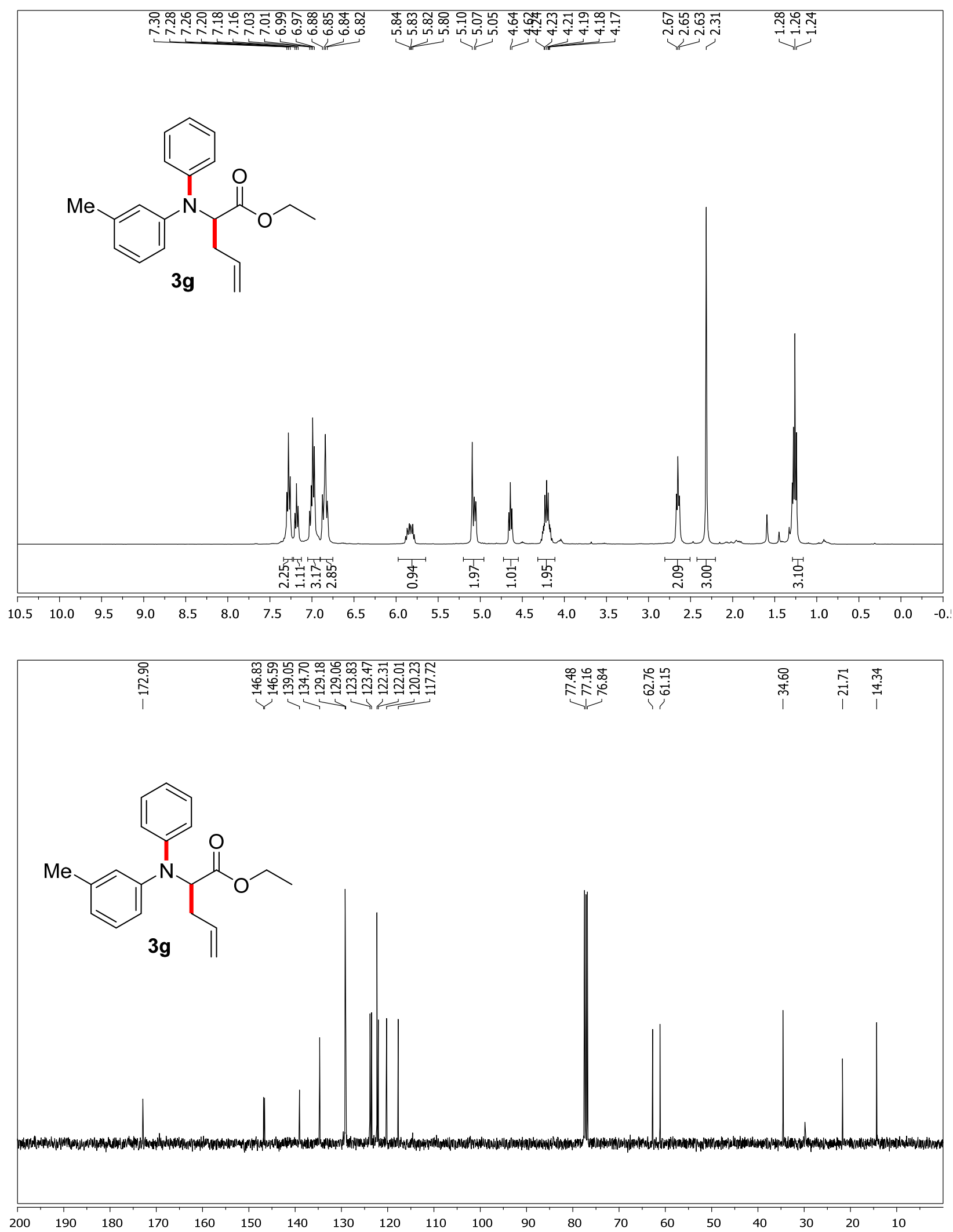
Ethyl 2-((3-chlorophenyl)(phenyl)amino)pent-4-enoate (3h)

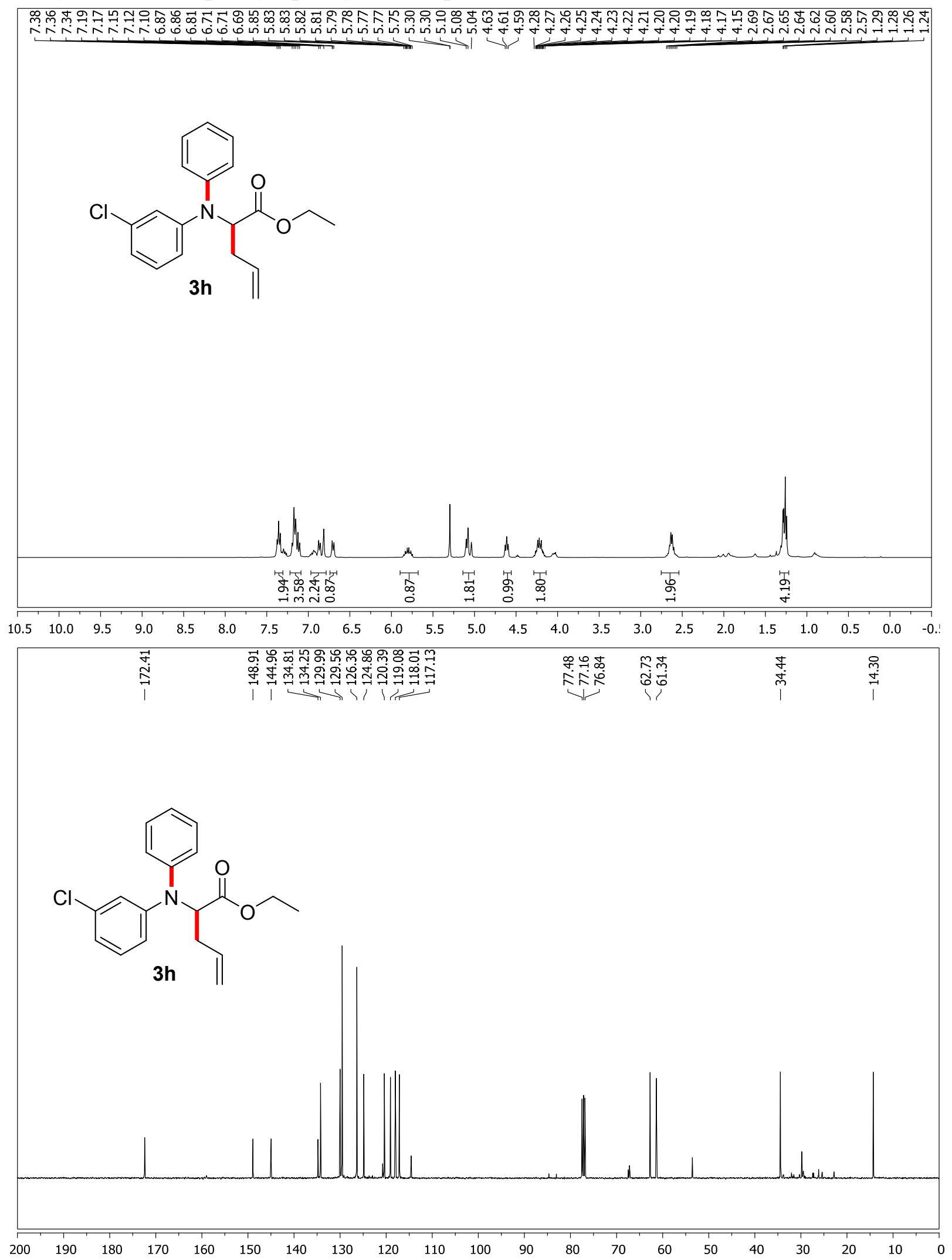


Ethyl-2-((3-bromophenyl)(phenyl)amino)pent-4-enoate (3i)

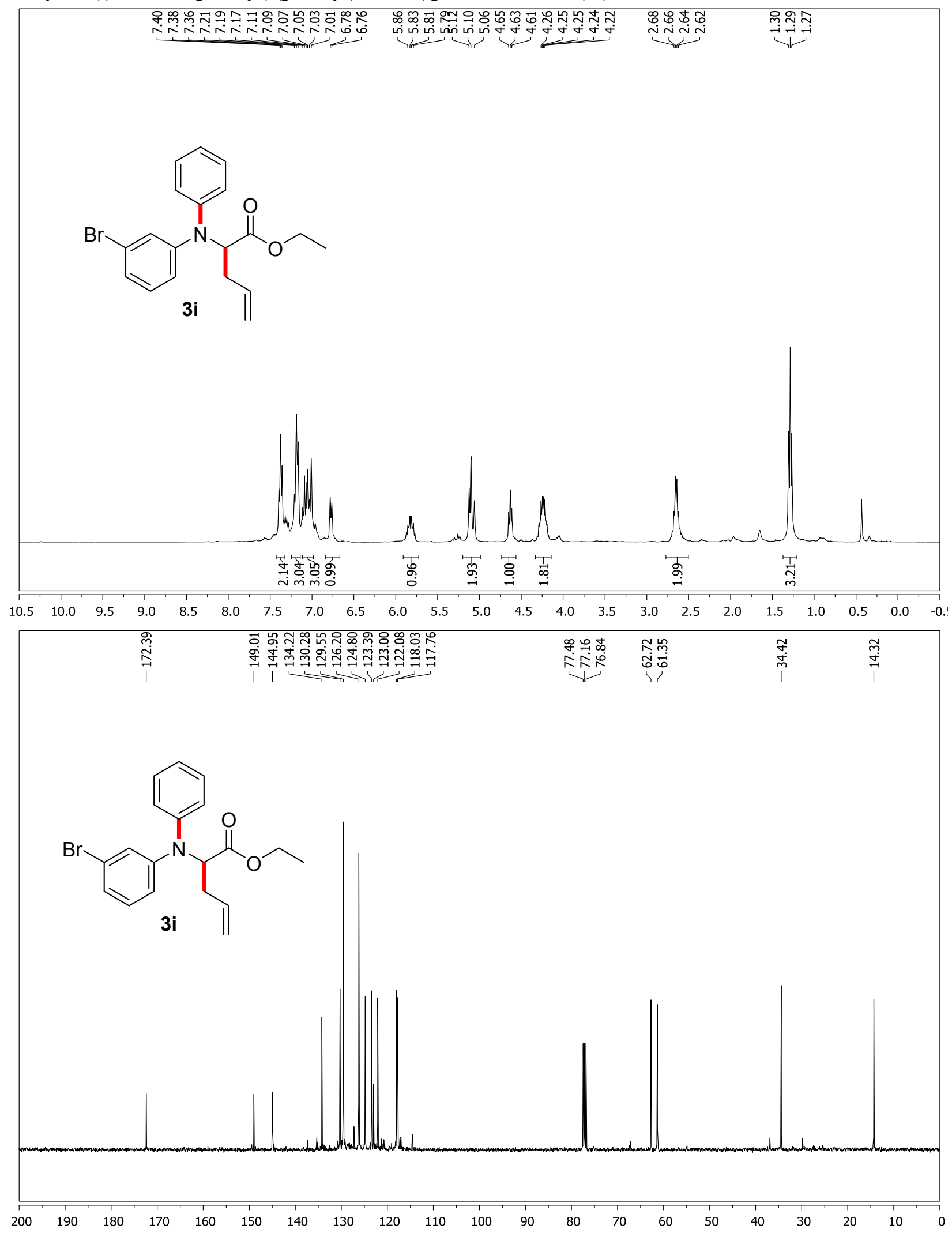


Ethyl-2-((3,4-dimethoxyphenyl)(phenyl)amino)pent-4-enoate (3j)

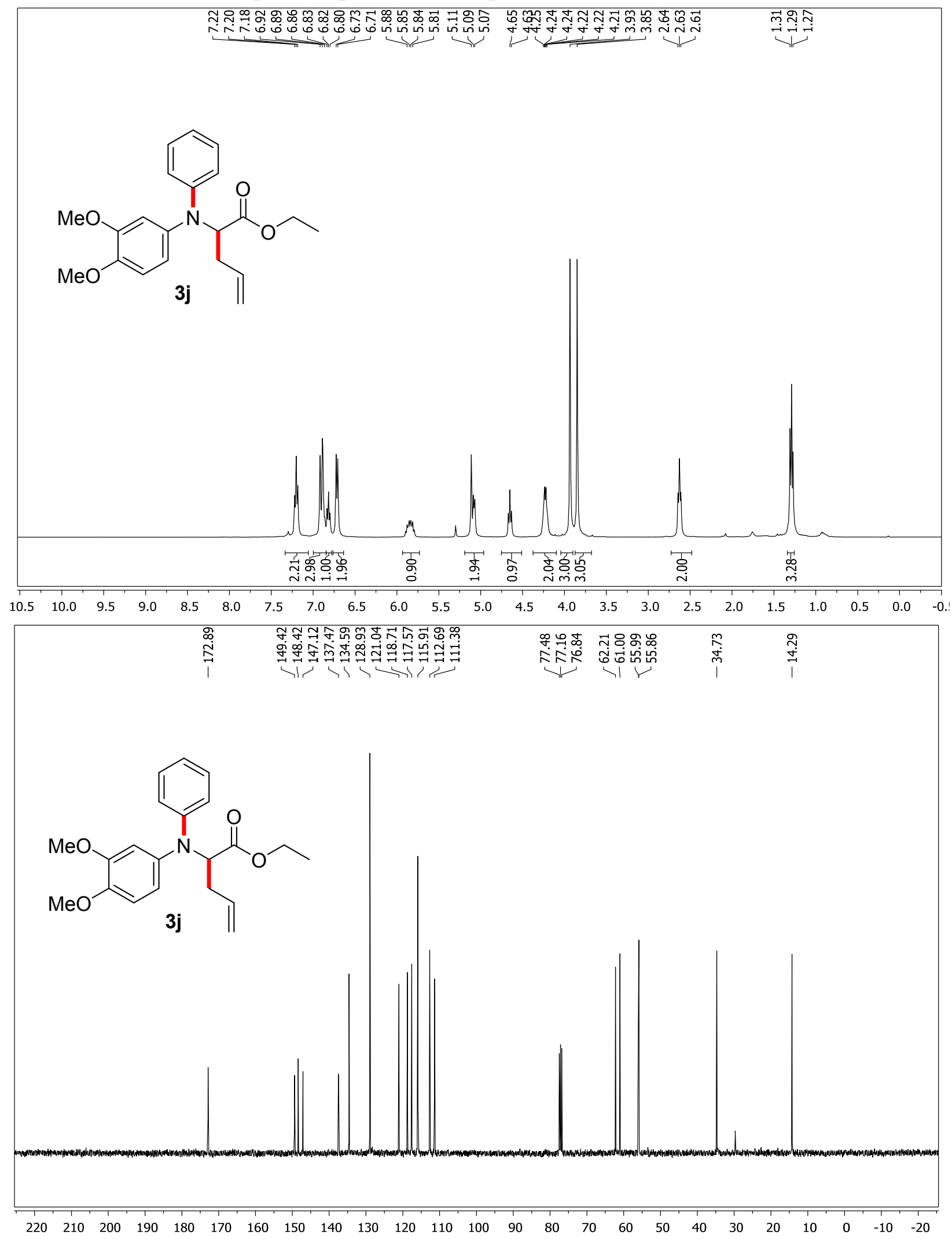


Ethyl-2-((3,4-difluorophenyl)(phenyl)amino)pent-4-enoate (3k)
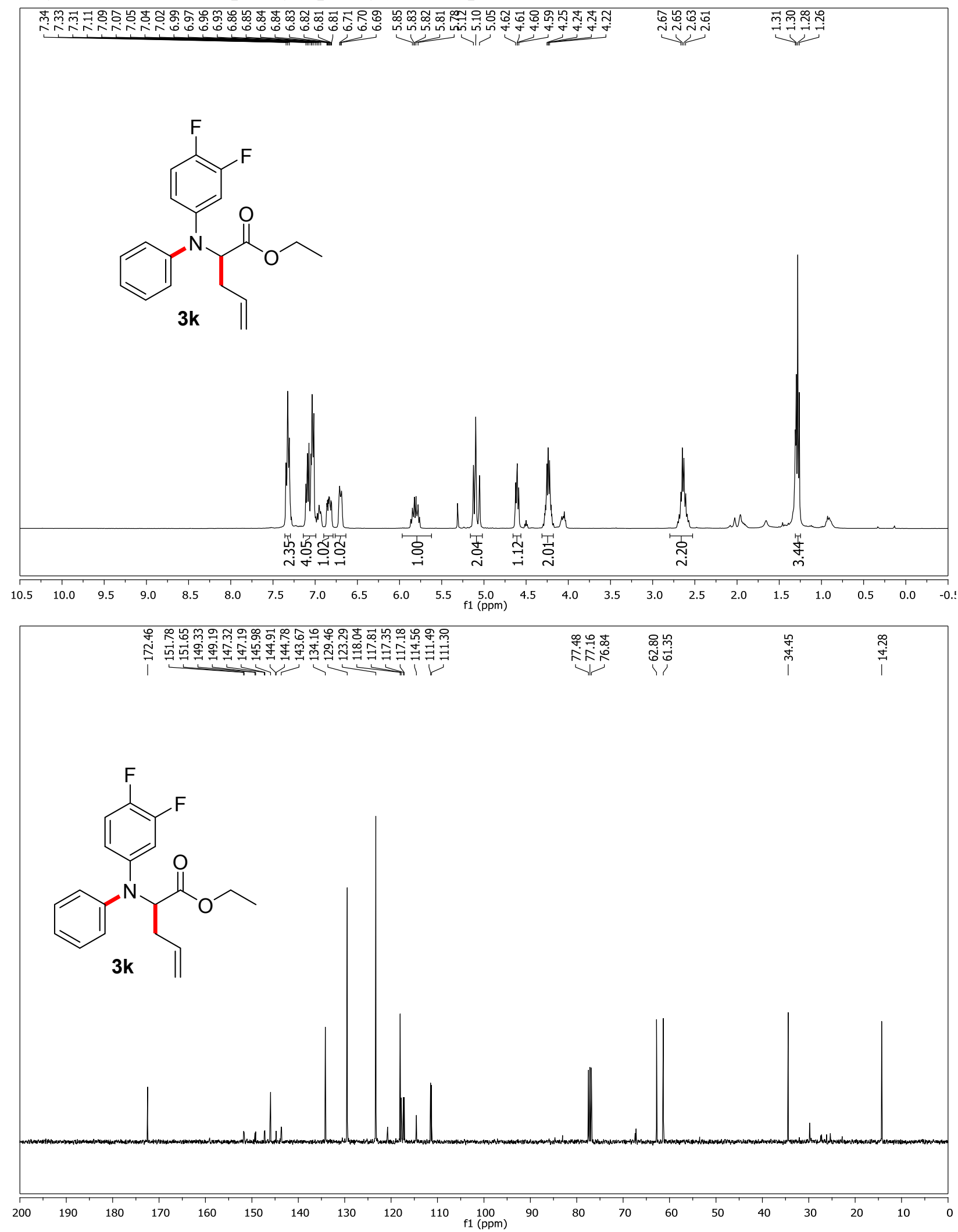
Ethyl 2-((3-chloro-4-fluorophenyl)(phenyl)amino)pent-4-enoate (3I)

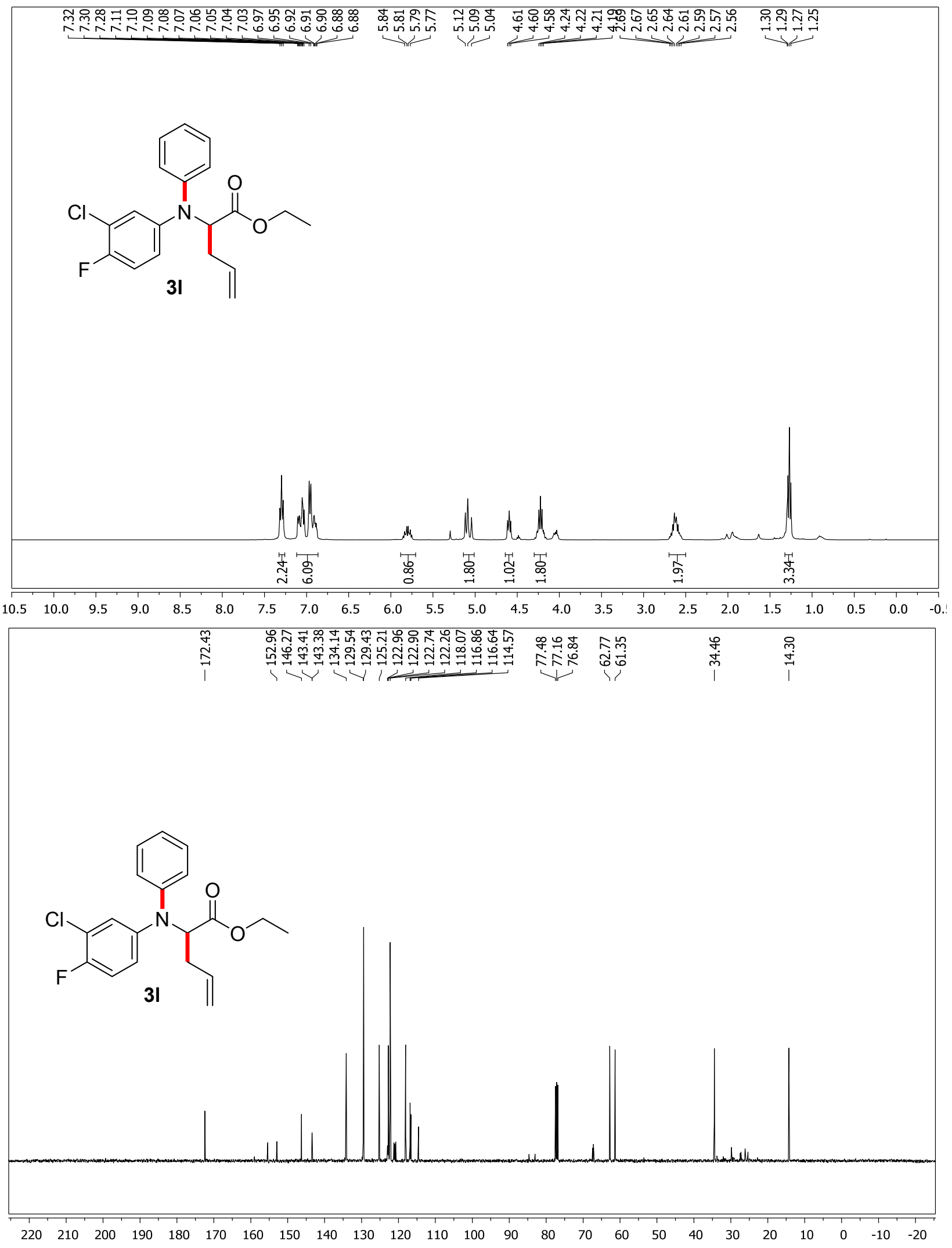

S36 
Ethyl 2-(phenyl(3,4,5-trimethoxyphenyl)amino)pent-4-enoate (3m)

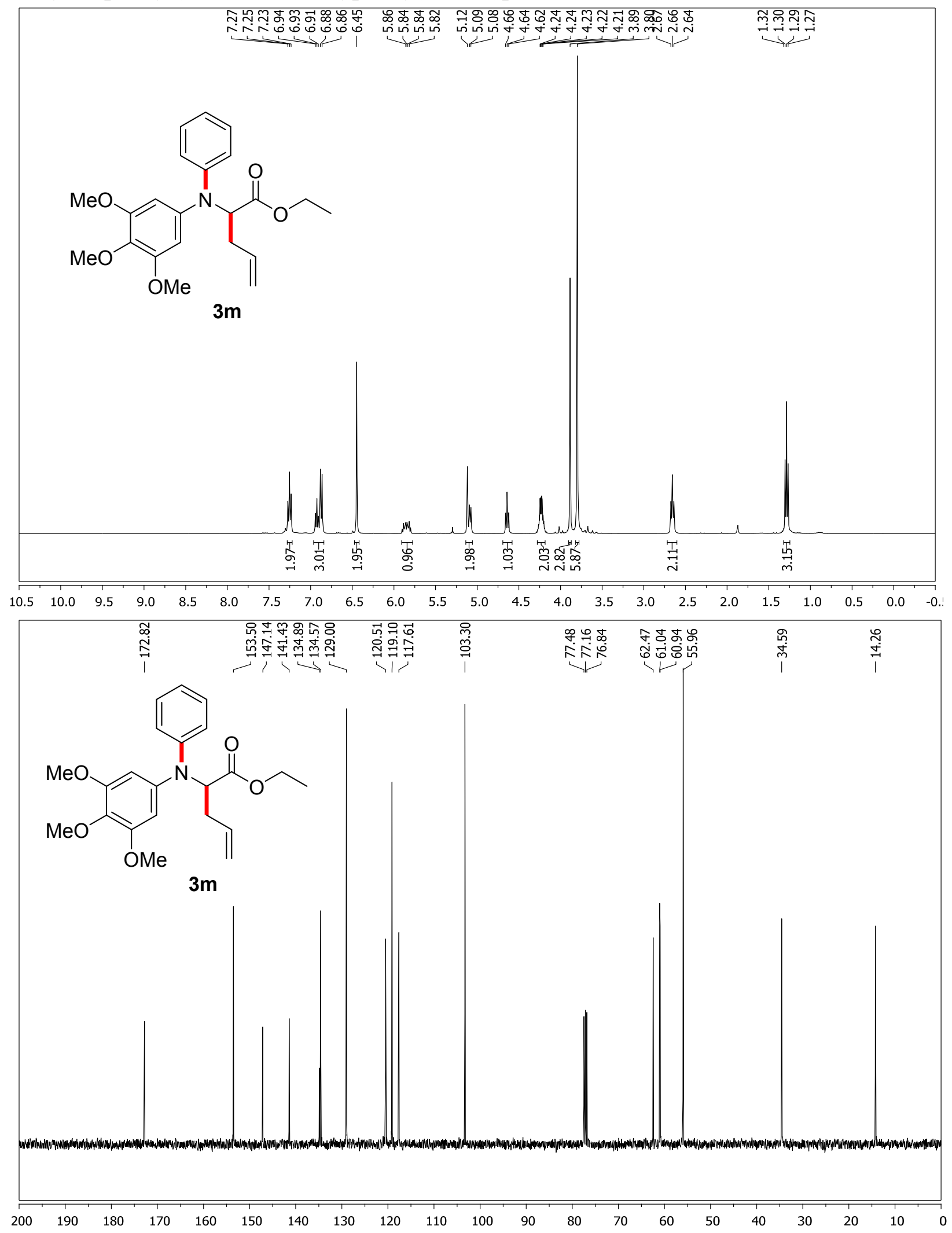




\section{2-(Diphenylamino)pent-4-enenitrile (3n)}
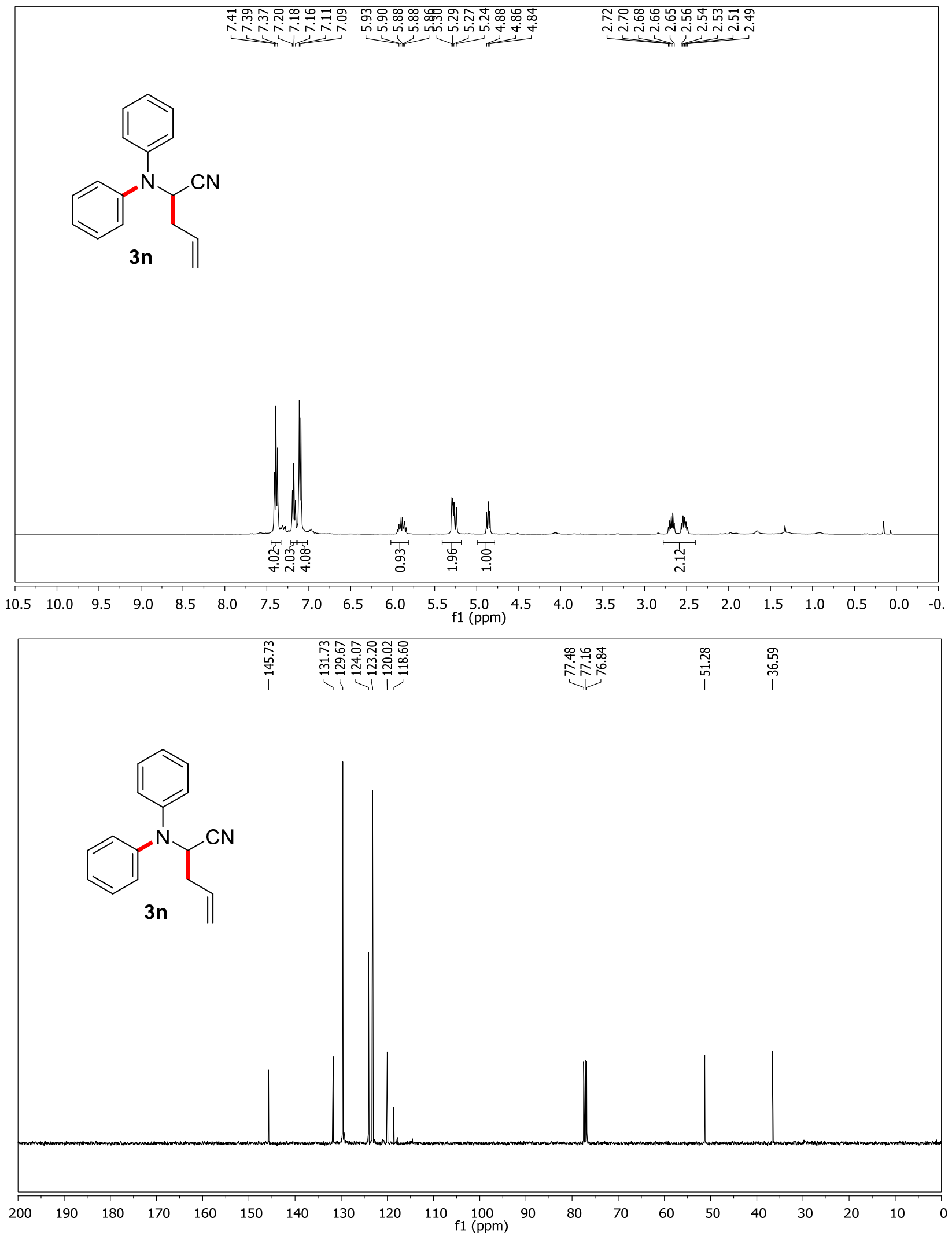
Methyl-2-(diphenylamino)pent-4-enoate (3o)
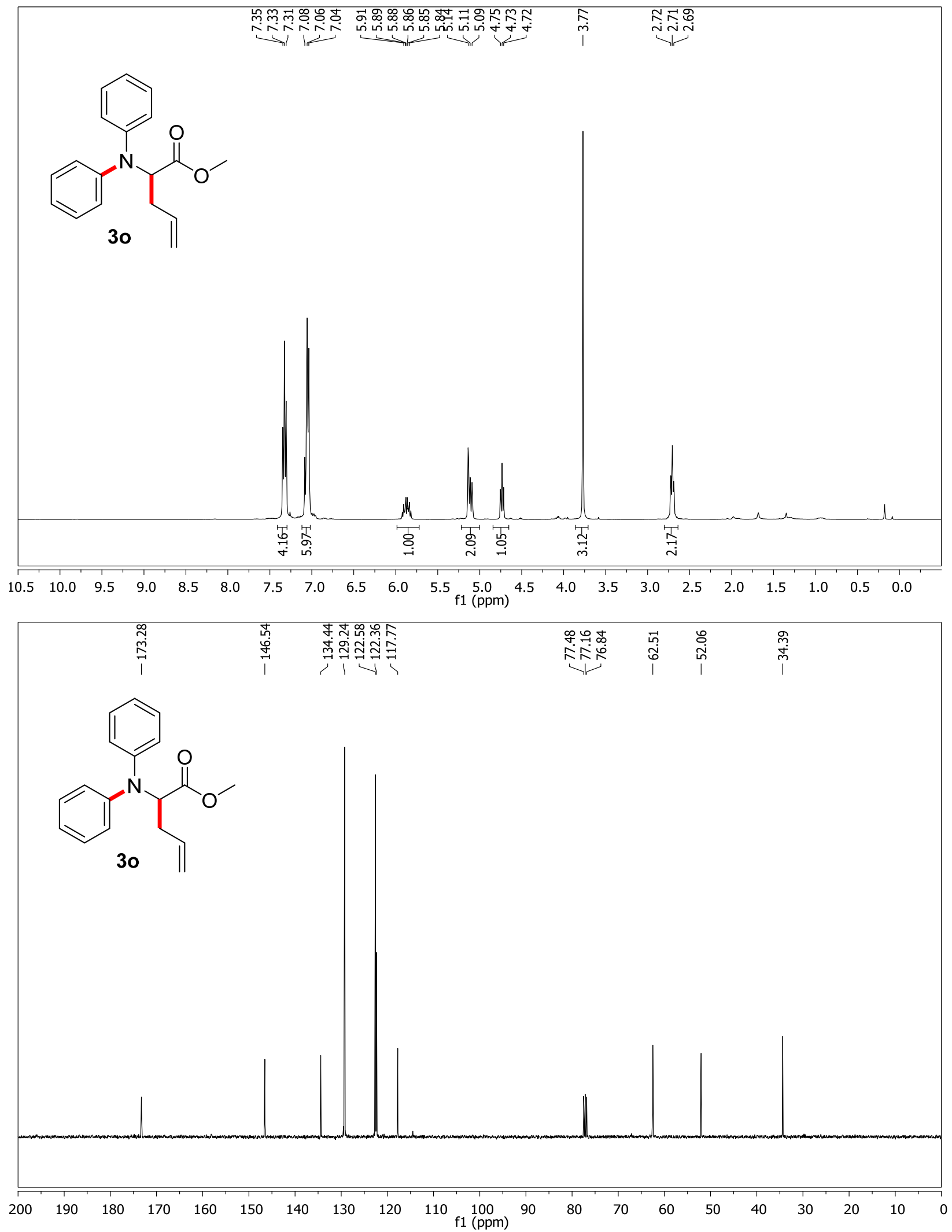


\section{Benzyl 2-(diphenylamino)pent-4-enoate (3p)}
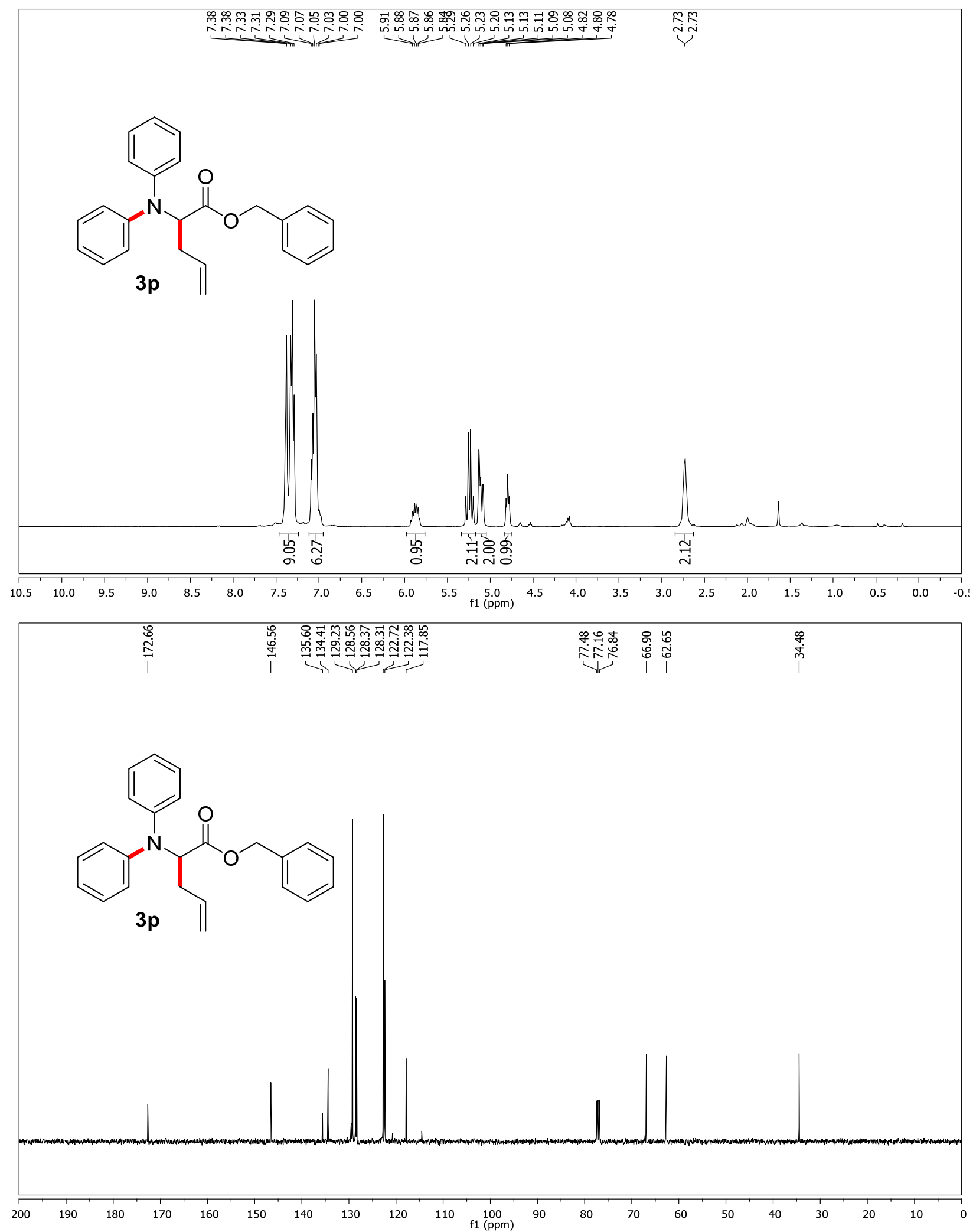
Tert-butyl 2-(diphenylamino)pent-4-enoate (3q)
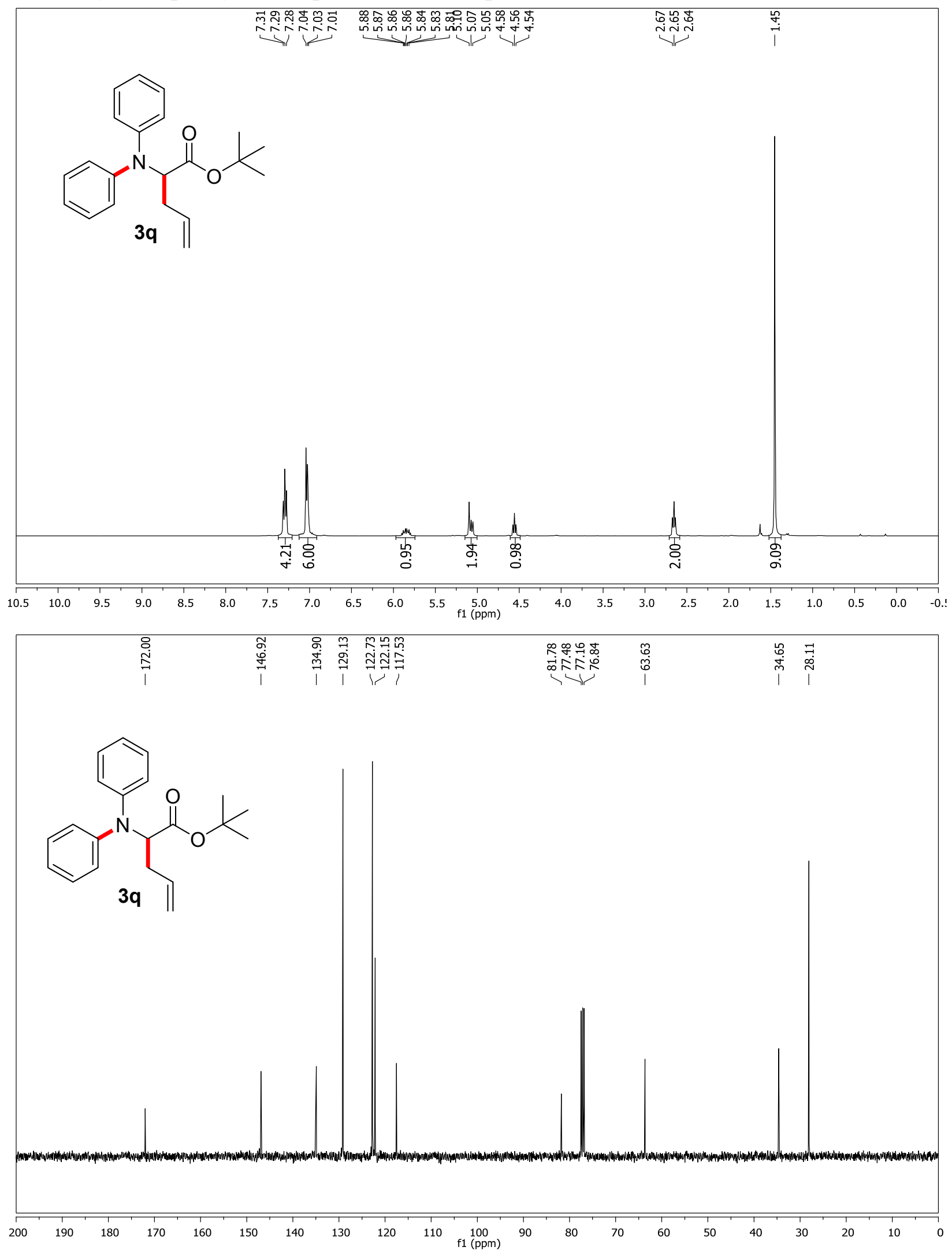

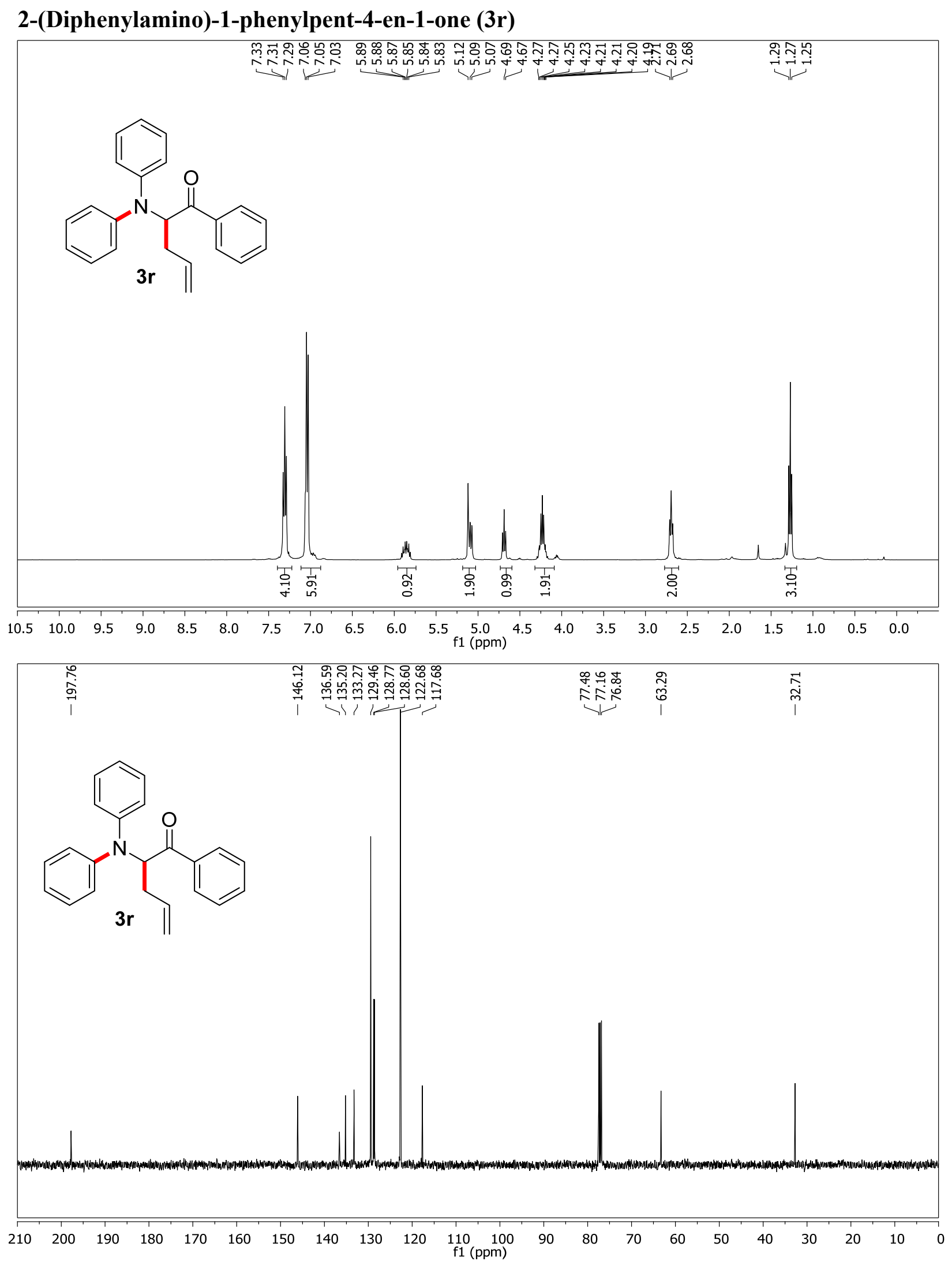

S42 


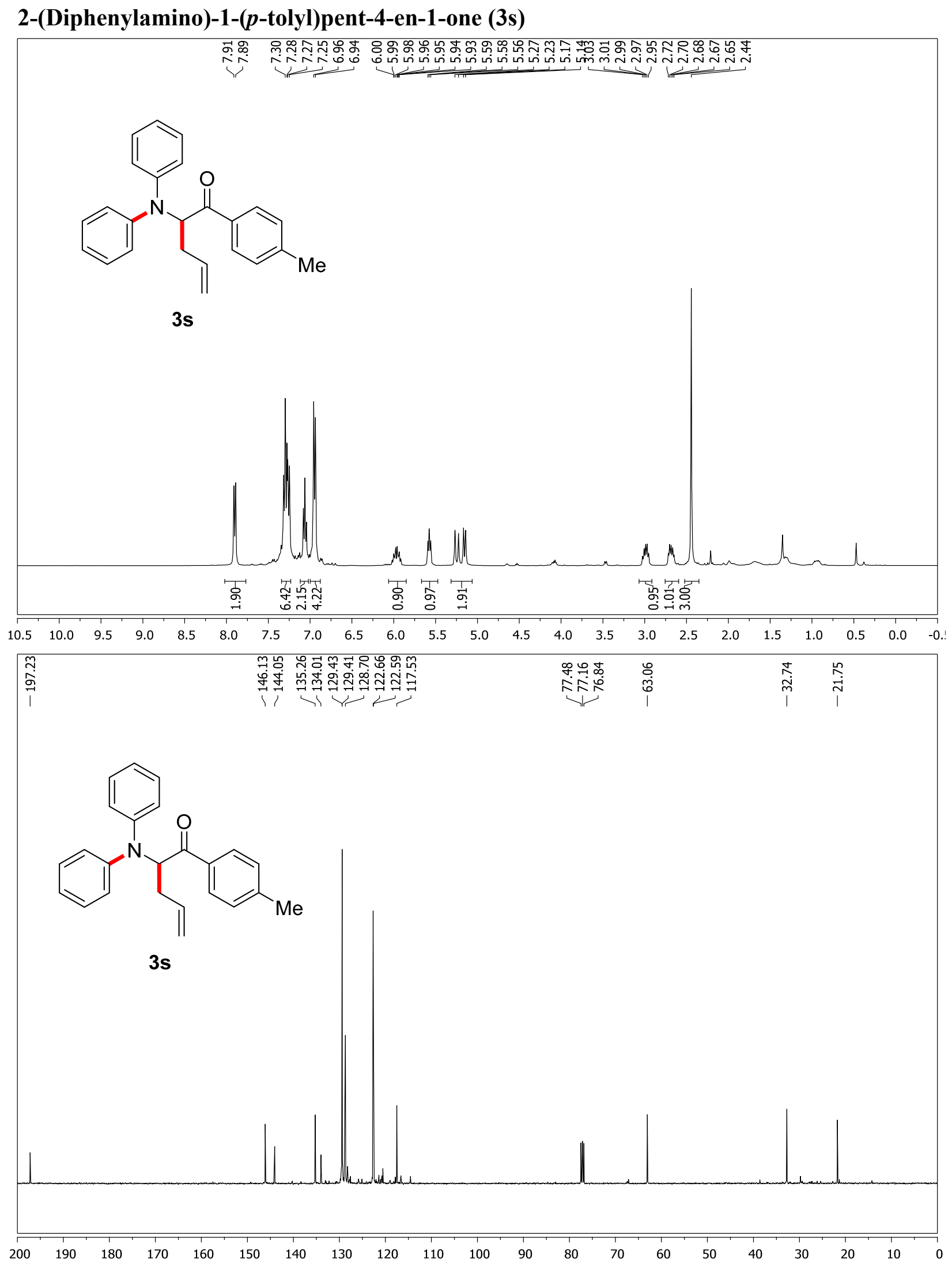

S43 


\section{$N$-(1-(4-bromophenyl)but-3-en-1-yl)- $N$-phenylaniline (3t)}
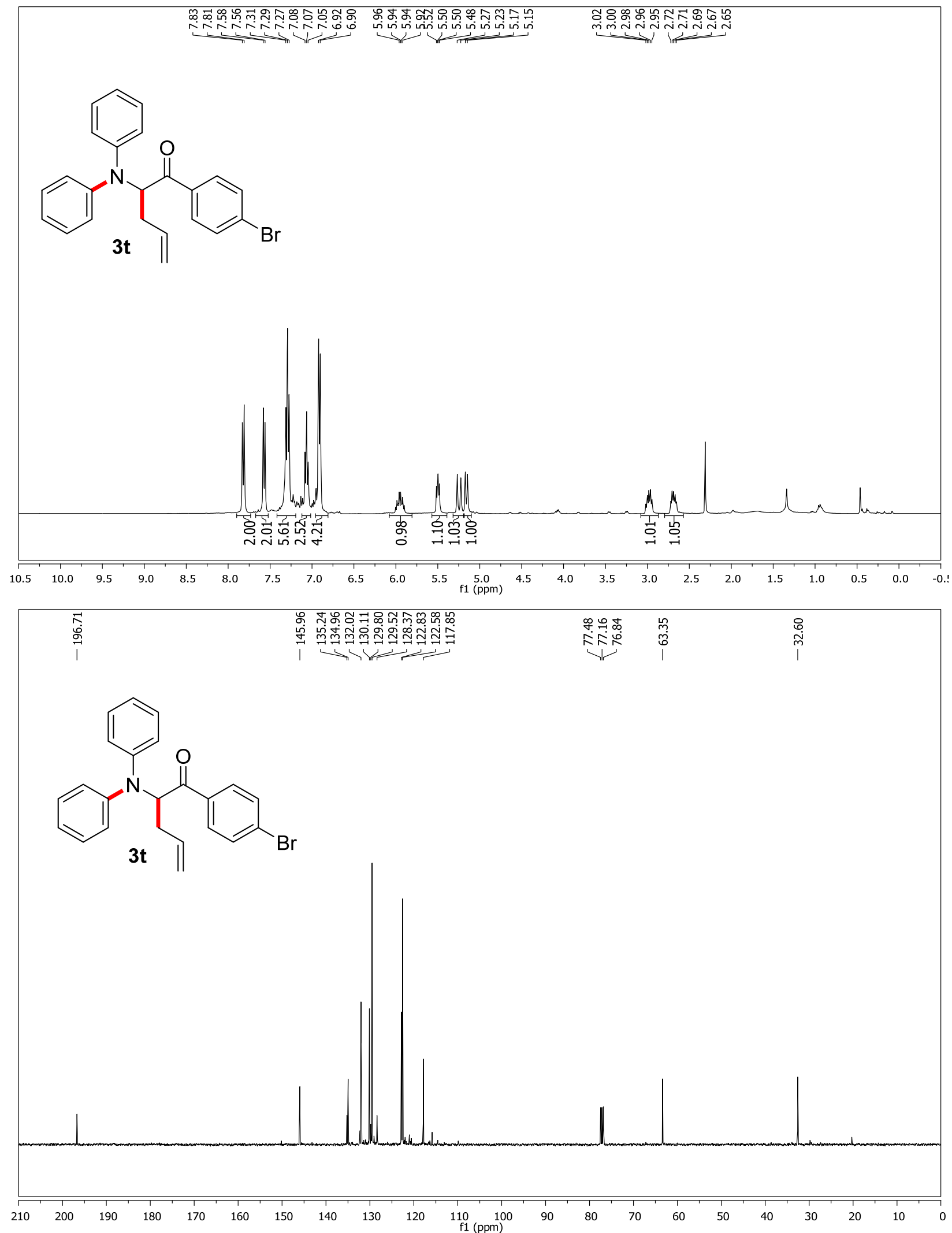
Ethyl 2-((3,4-dimethylphenyl)(phenyl)amino)pent-4-enoate (3u)
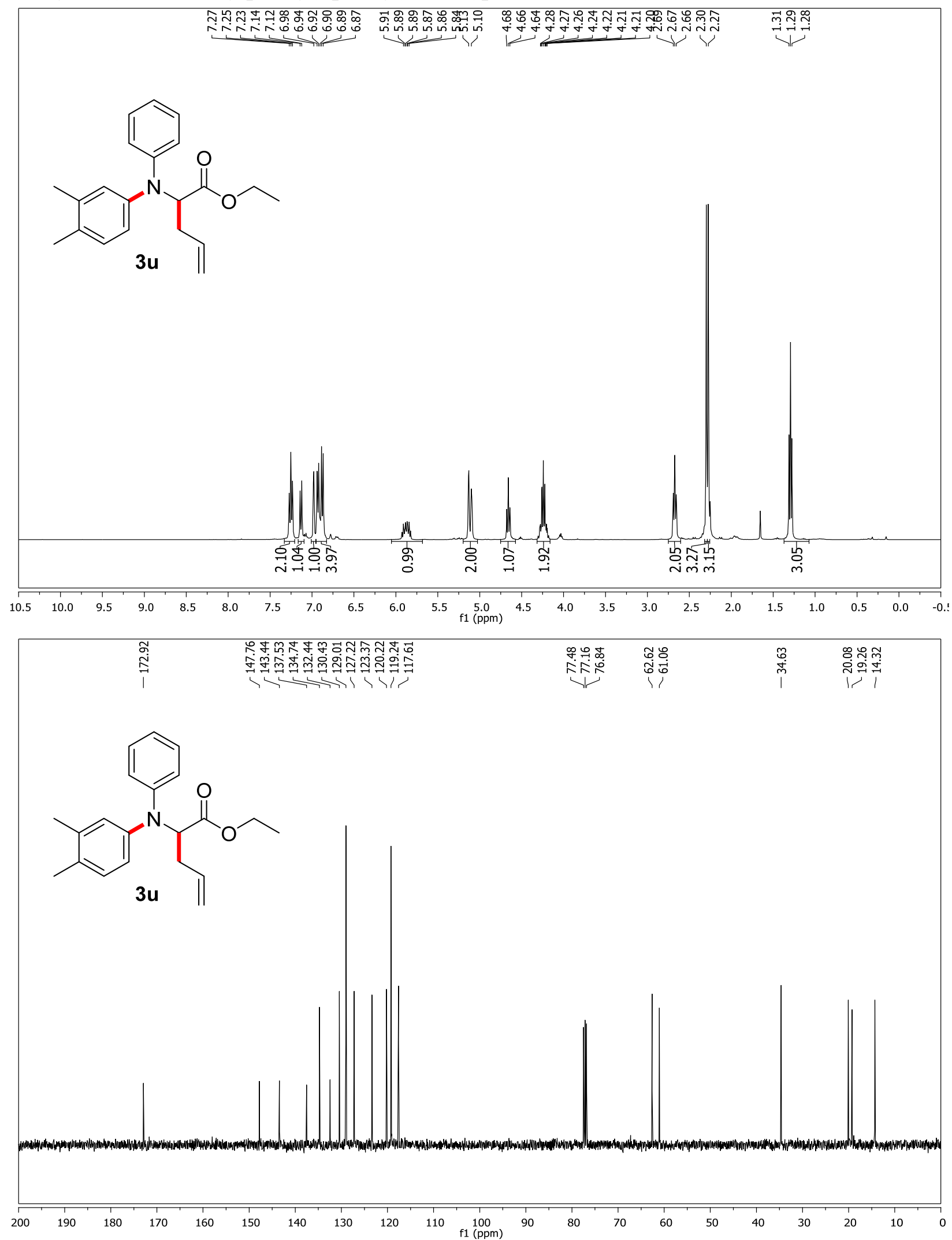
Ethyl-2-(benzo[d][1,3]dioxol-5-yl(phenyl)amino)pent-4-enoate (3v)

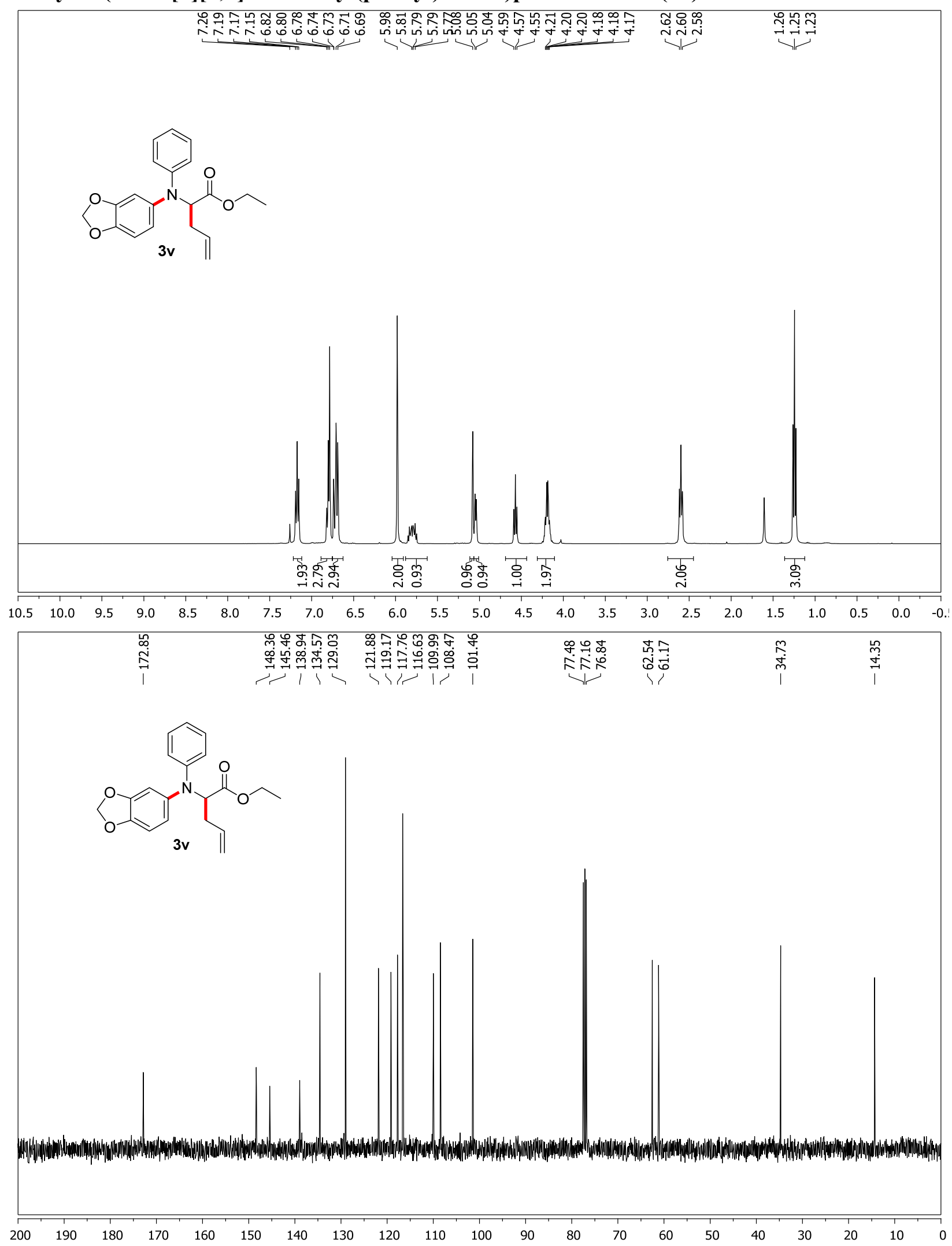




\section{Ethyl 2-((3,4-difluorophenyl)(phenyl)amino)pent-4-enoate (3w)}
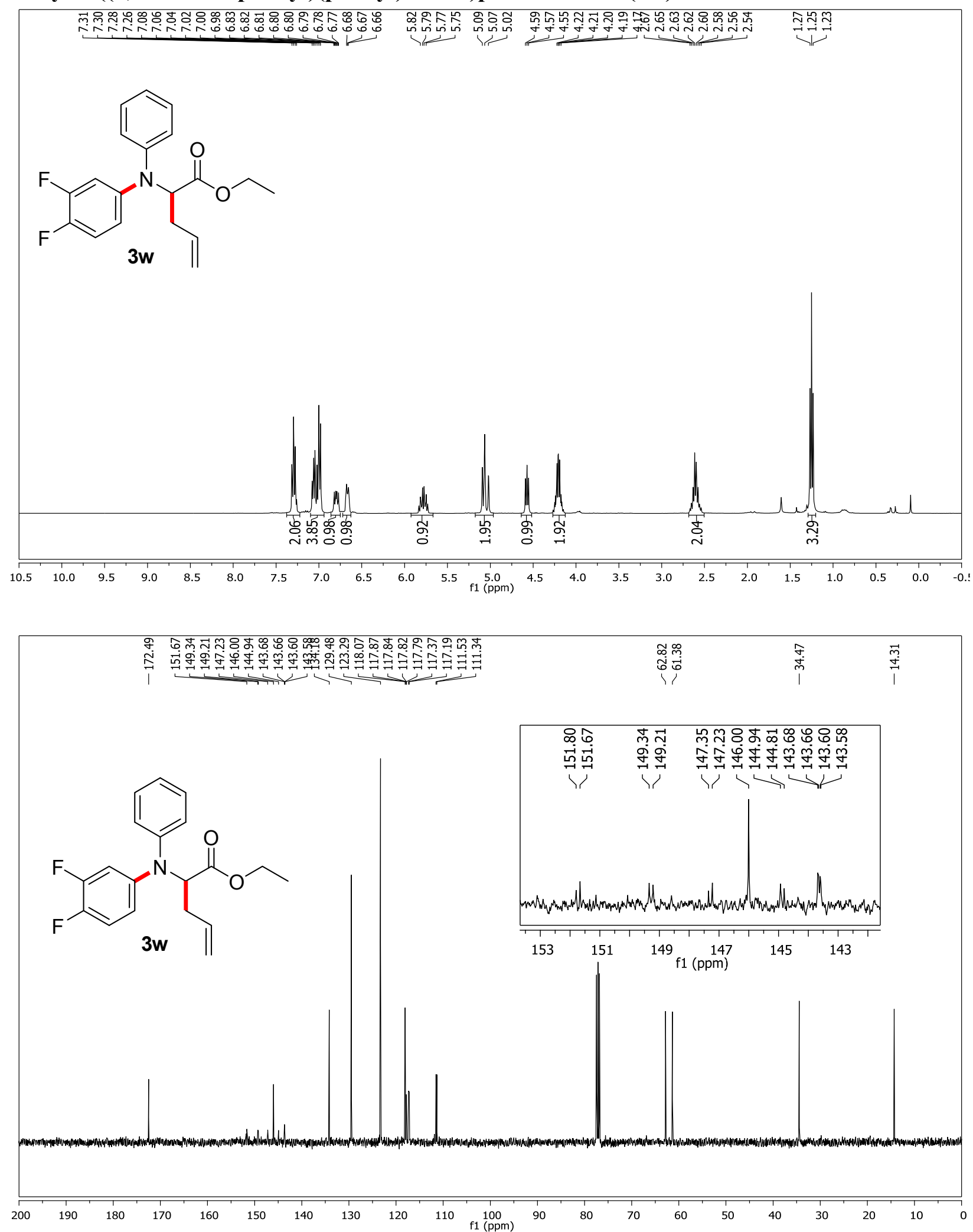
Ethyl 2-(naphthalen-2-yl(phenyl)amino)pent-4-enoate (3x)
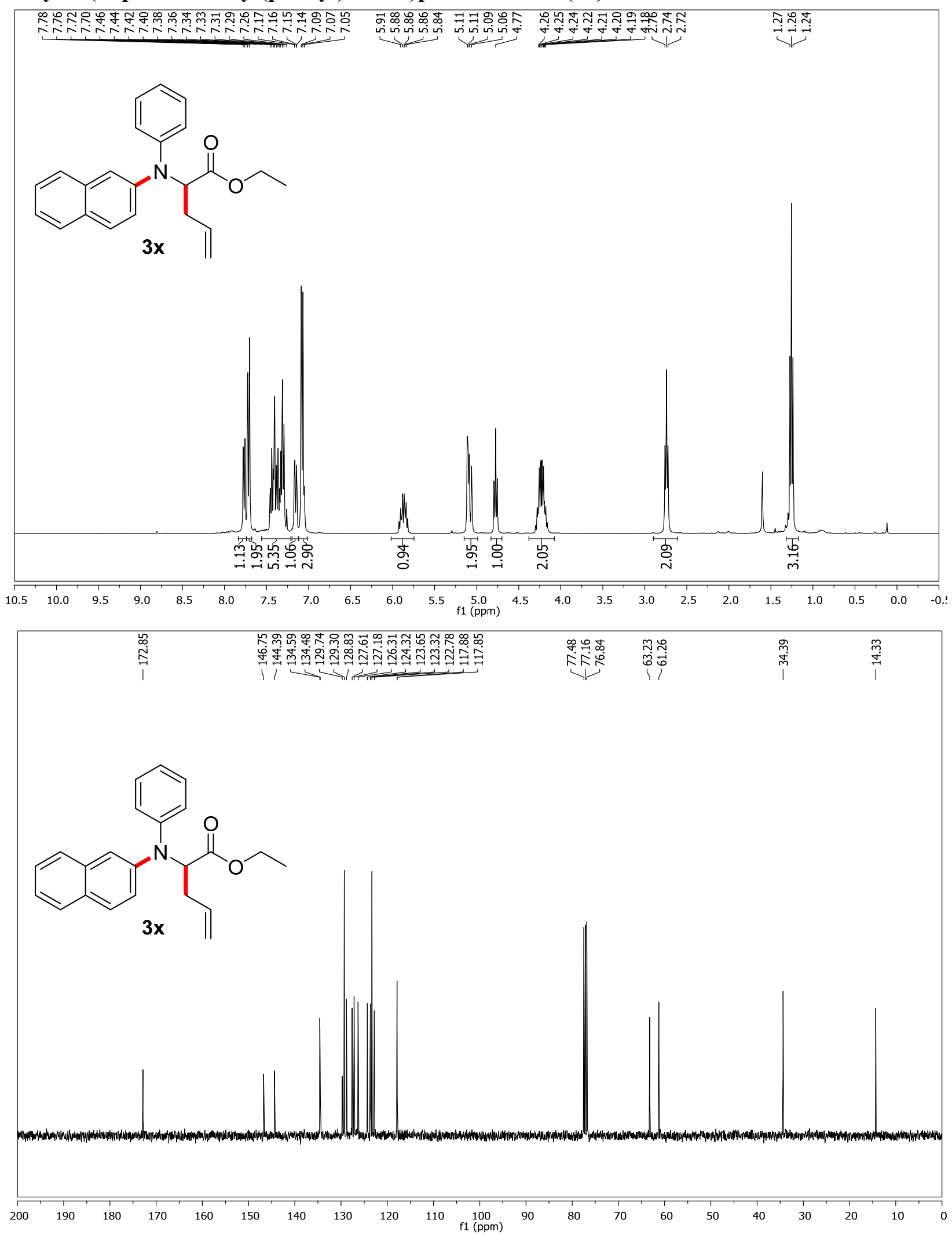
Ethyl-2-(phenyl(m-tolyl)amino)pent-4-enoate (3y) and Ethyl-2-(phenyl(p-tolyl)amino)pent4-enoate (3y')
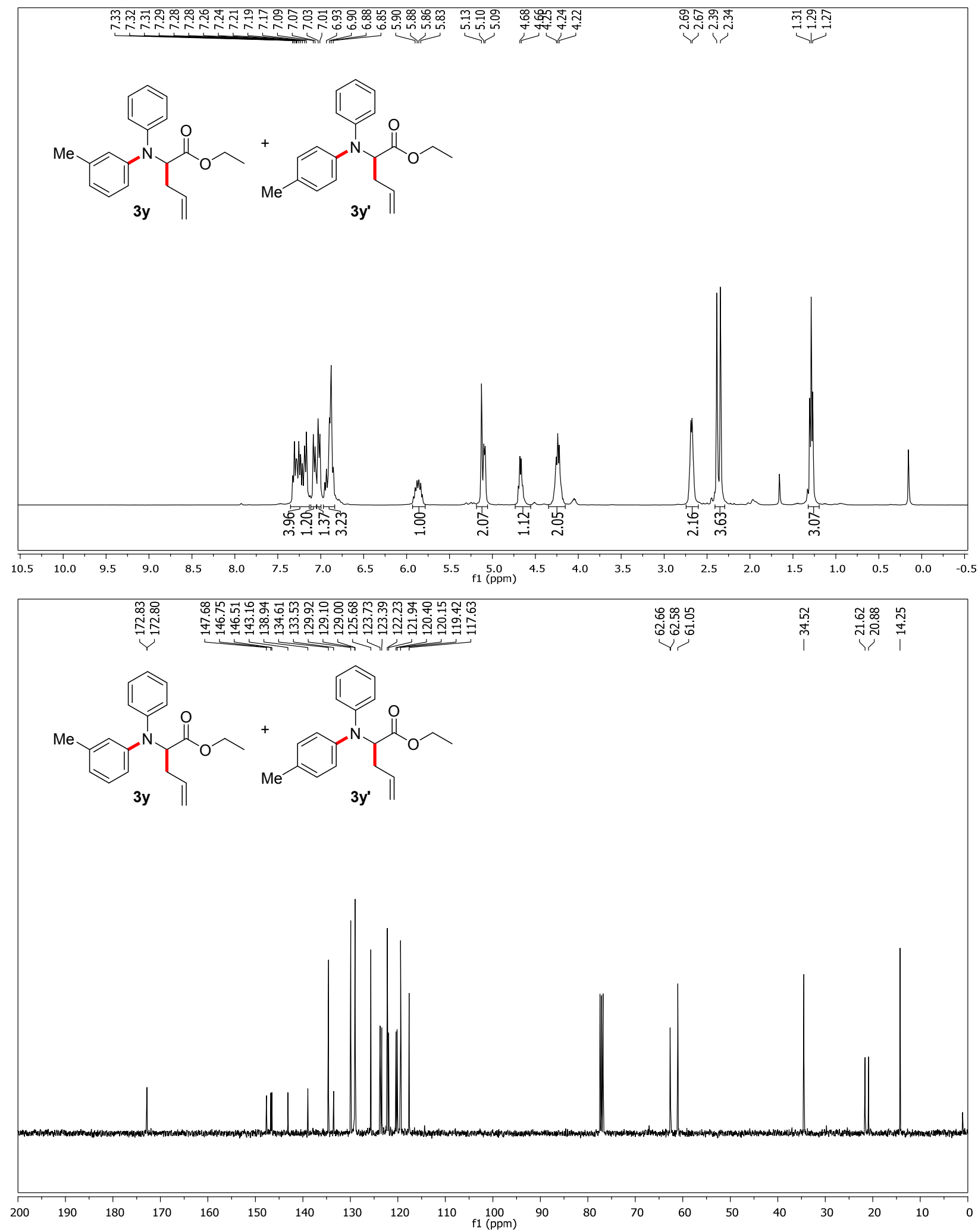
Ethyl-2-((4-fluorophenyl)(phenyl)amino)pent-4-enoate (3z) and

Ethyl-2-((3 fluorophenyl)(phenyl)amino)pent-4-enoate (3z')

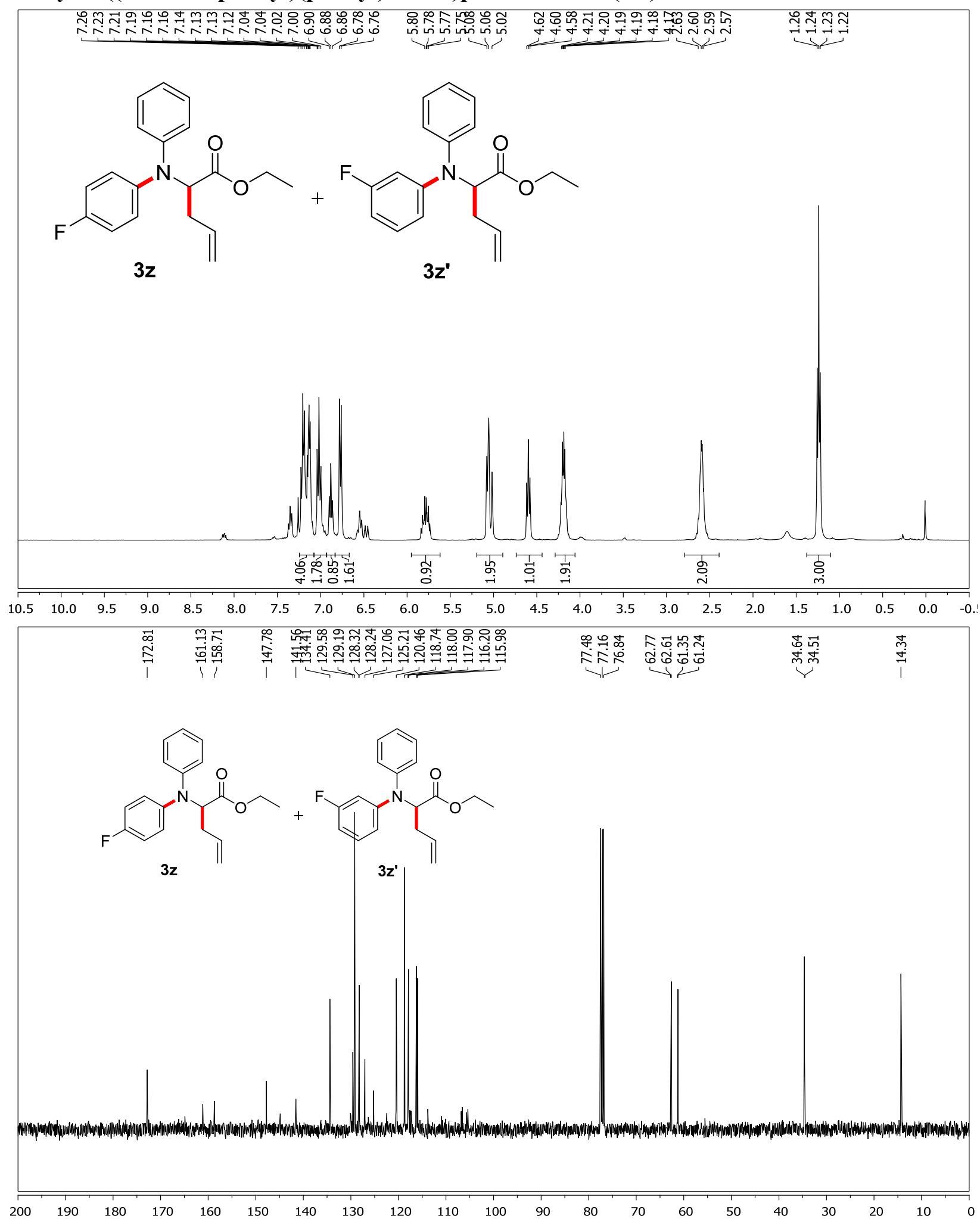


Ethyl 2-(diphenylamino)-3-phenylpent-4-enoate (4)
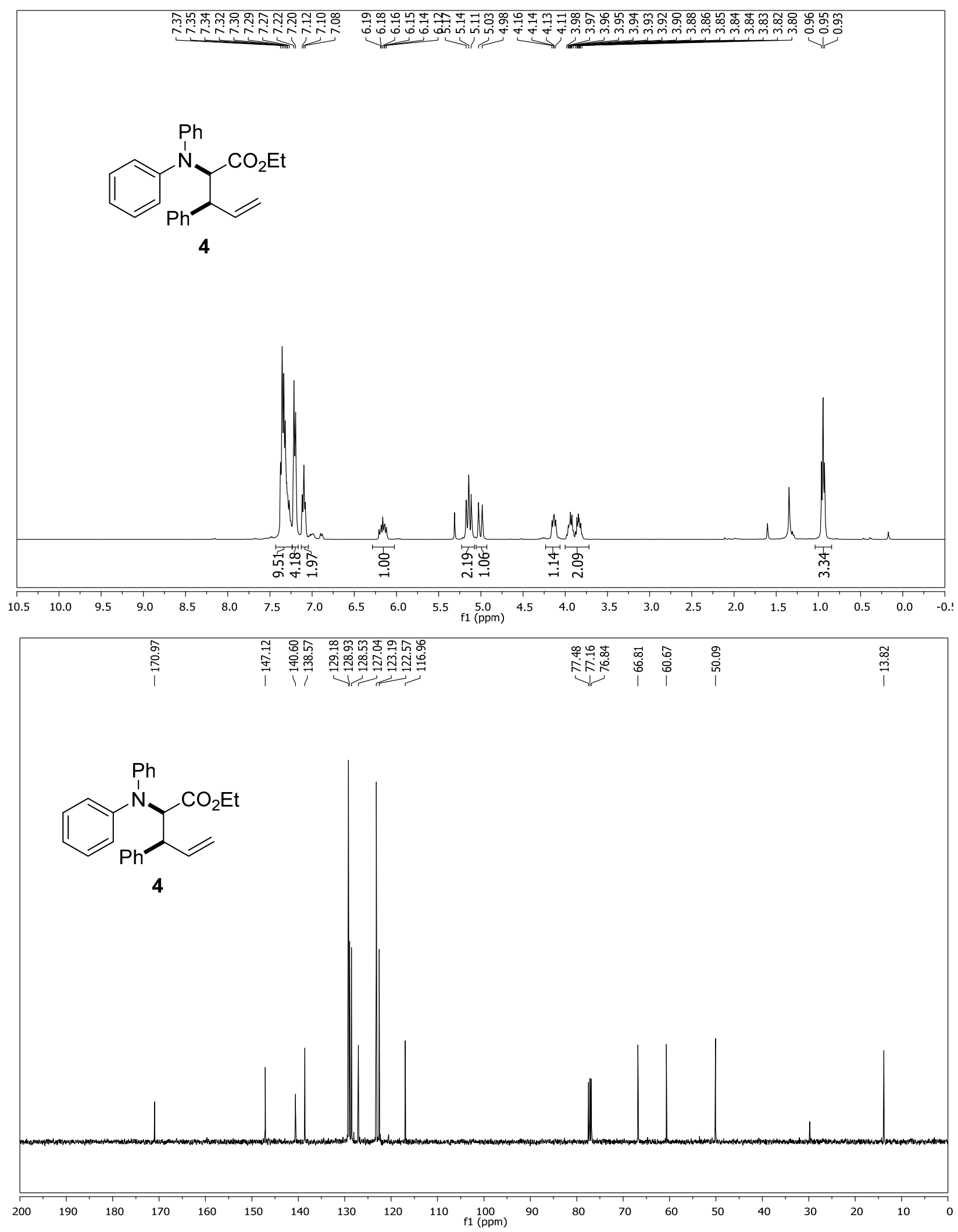
Ethyl-2-(diphenylamino)-3-methylpent-4-enoate (5)

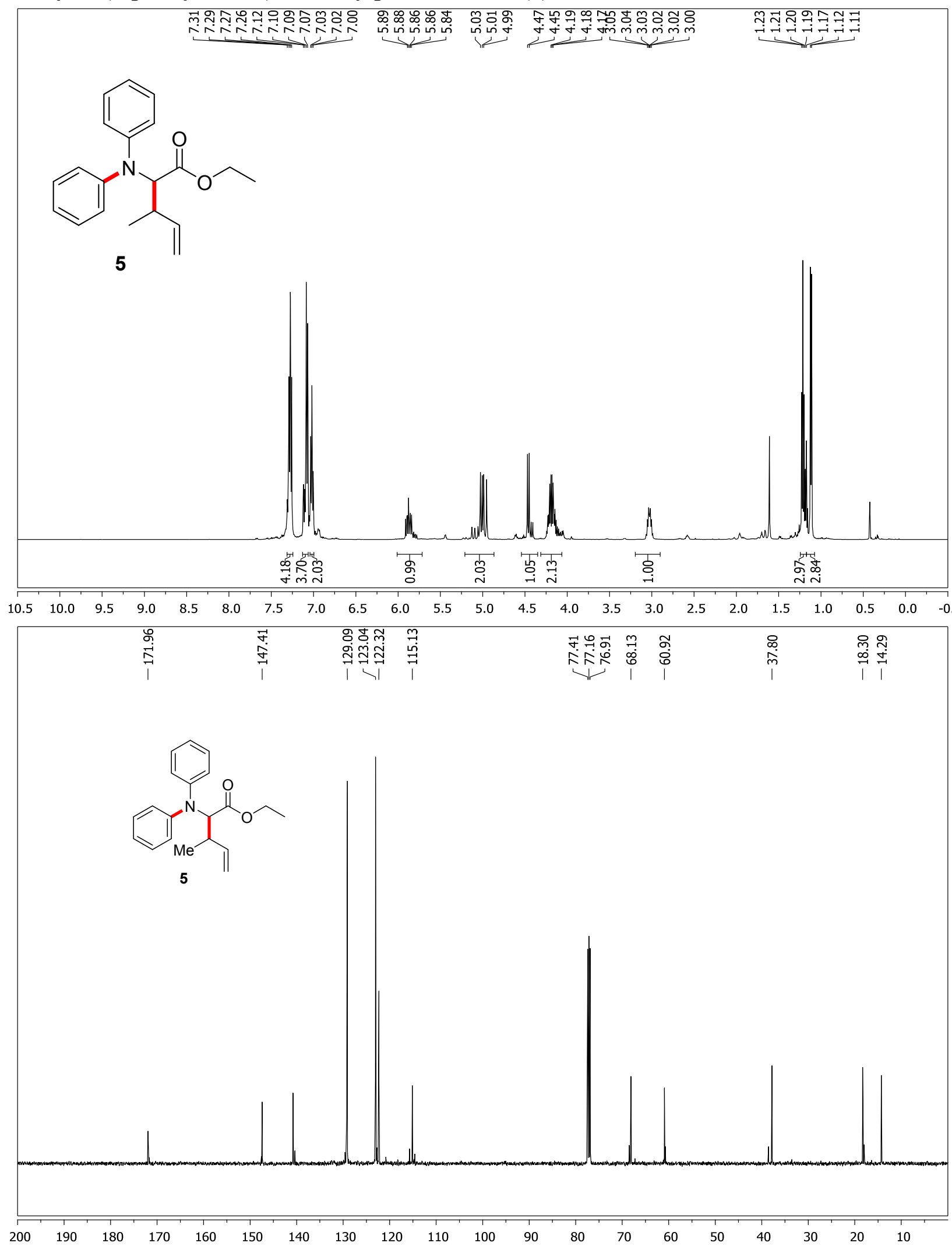


Methyl (S)-2-allyl-1-phenylpyrrolidine-2-carboxylate (7a)
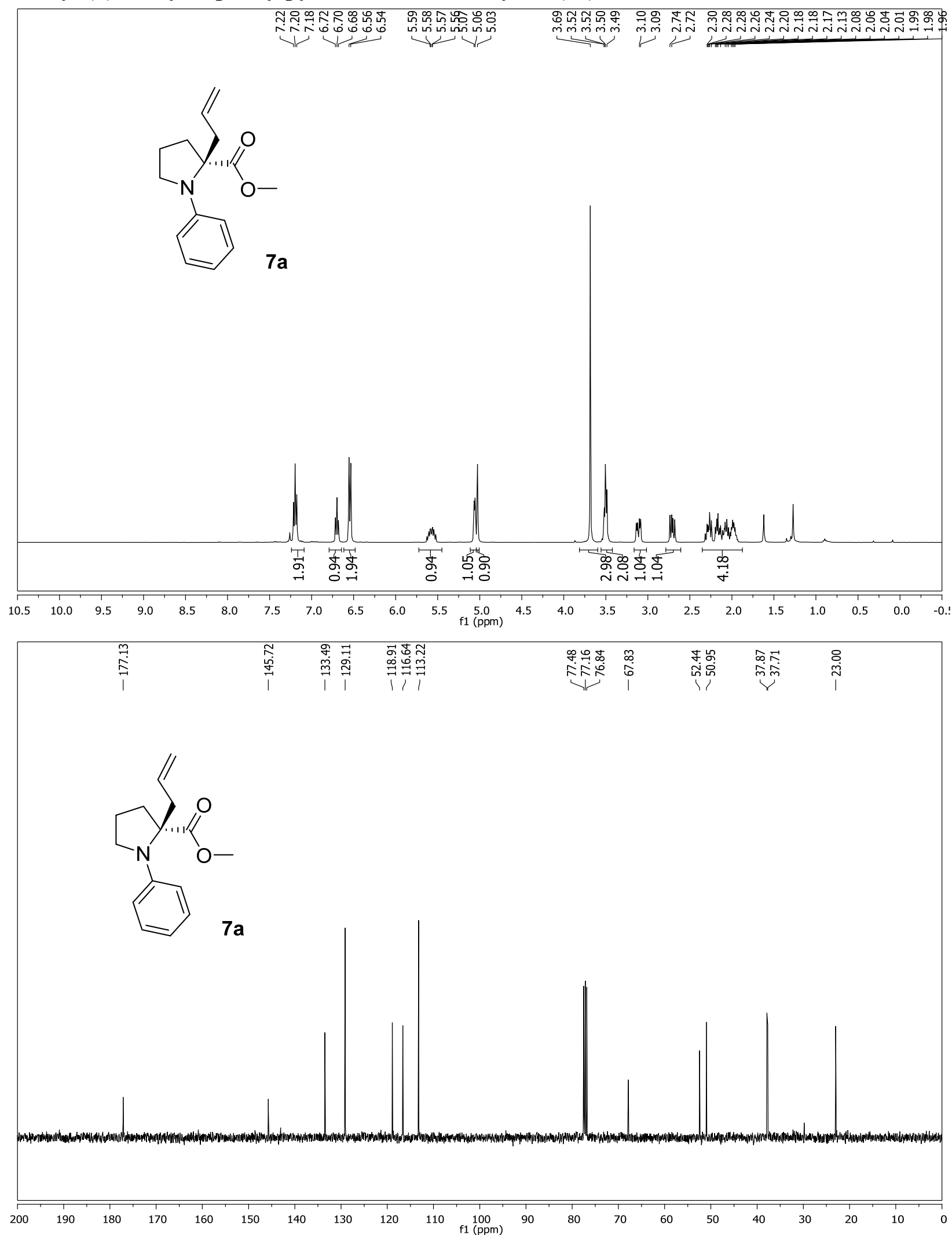
(Ethyl (S)-2-allyl-1-phenylpyrrolidine-2-carboxylate (7b)
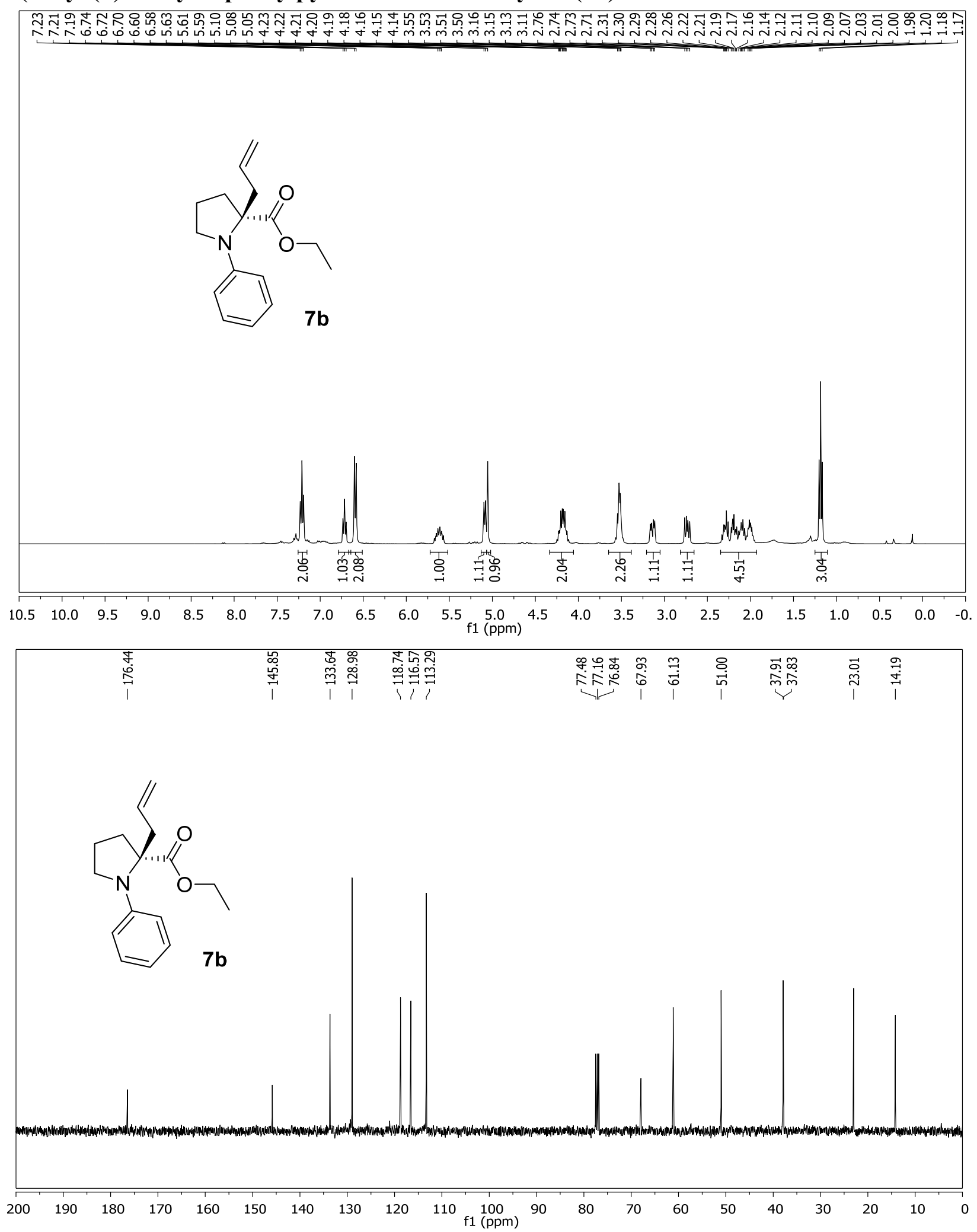
Methyl (S)-2-allyl-1-(benzo[d][1,3]dioxol-5-yl)pyrrolidine-2-carboxylate (7c)
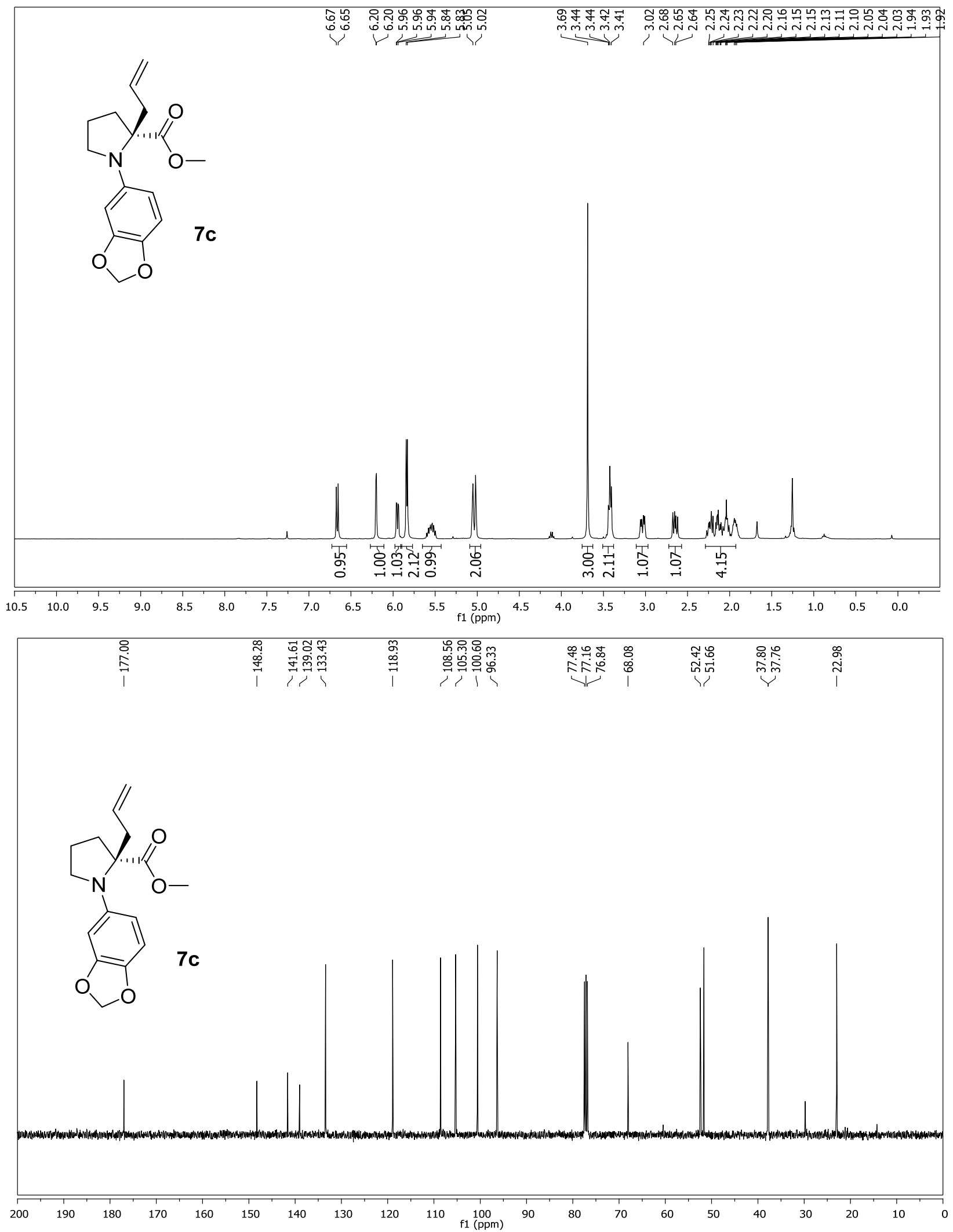


\section{Ethyl diphenyl-phenylalaninate (9)}
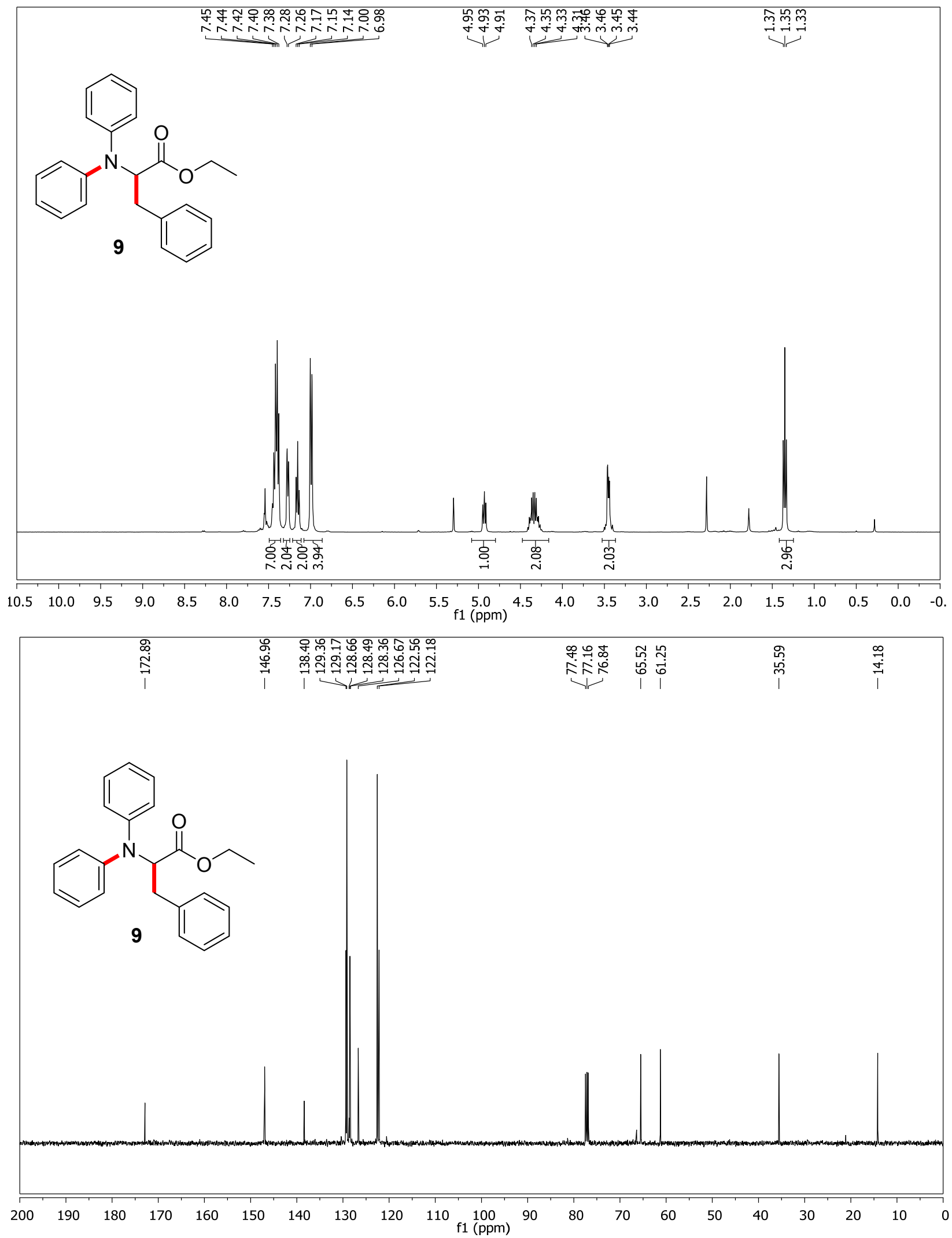


\section{HPLC data of Chiral Proline Derivatives}

\section{Methyl (S)-2-allyl-1-phenylpyrrolidine-2-carboxylate (7a)}

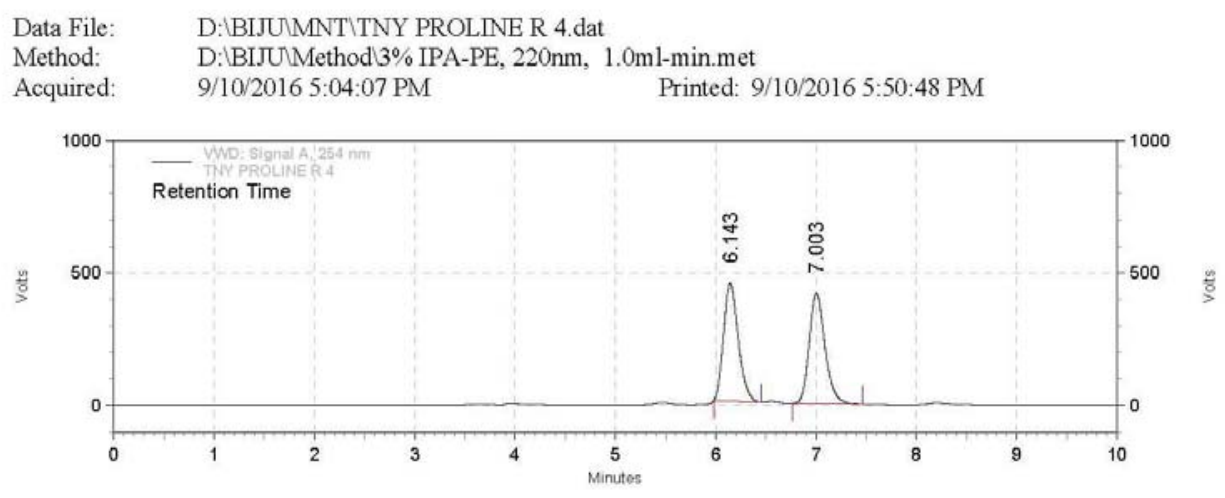

VWD: Signal A, $254 \mathrm{~nm}$ Results

\begin{tabular}{rrrr|}
$r$ Retention Time & Area & Area \% \\
\hline 6.143 & 75848983 & 49.76 \\
7.003 & 76574359 & 50.24 \\
\hline \multicolumn{2}{|r|}{ Totals } & & \\
\hline & & 152423342 & 100.00 \\
\hline
\end{tabular}

Column : $\quad$ Chiralcel OD-H

Eluent System : $98: 2$ (HEXANE:IPA)

Flow rate: $\quad 0.8 \mathrm{ml} / \mathrm{min}$

Injection vol..: $10 \mathrm{ul}$

Wavelength: $254 \mathrm{~nm}$

Sample Conc.: $1 \mathrm{mg} / \mathrm{ml}$

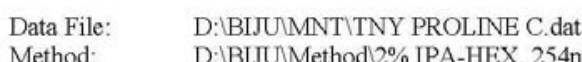

Method: D: DIJUlMethod $2 \%$ IPA-HEX, 254nm, $0.8 \mathrm{ml}$-min.met

Acquired: 9/10/2016 5:17:37 PM

Printed: 9/10/2016 5:46:47FI1

$7 a$

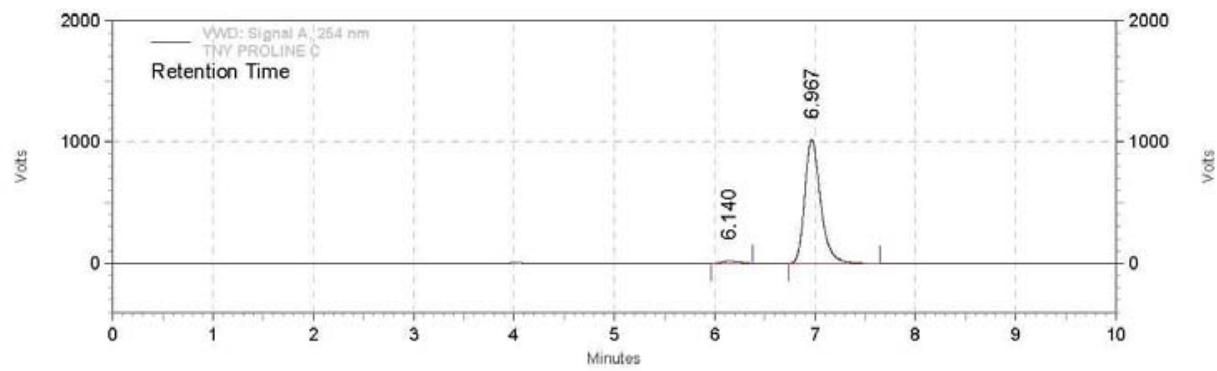

VWD: Signal A, $254 \mathrm{~nm}$ Results

Retention Time

Retention Time
6.140
6.967

Area

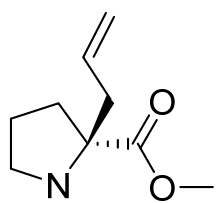

187008502

Area \%

Totals

\begin{tabular}{|r|r|r|}
\hline Totals & 191086656 & 100.00 \\
\hline
\end{tabular}


(Ethyl (S)-2-allyl-1-phenylpyrrolidine-2-carboxylate (7b)

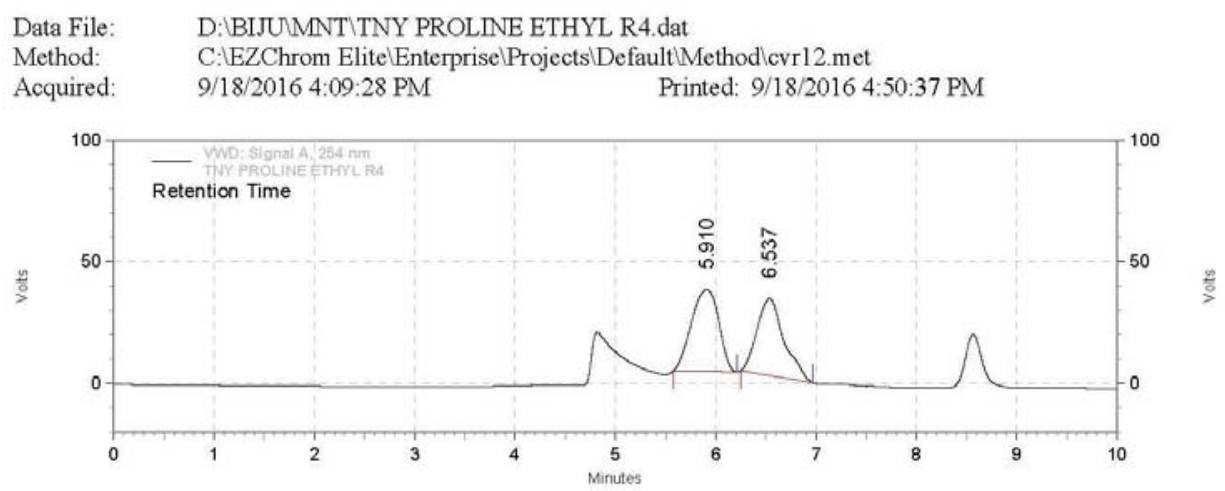

VWD: Signal A, $254 \mathrm{~nm}$ Results

\begin{tabular}{|c|c|c|}
\hline Retention Time & Area & Area $\%$ \\
\hline 5.910 & 10684226 & 51.82 \\
\hline 6.537 & 9933781 & 48.18 \\
\hline Totals & 20618007 & 100.00 \\
\hline
\end{tabular}

$\begin{array}{ll}\text { Column : } & \text { Chiralpak IB } \\ \text { Eluent System : } & 99: 1 \text { (HEXANE:IPA) } \\ \text { Flow rate: } & 0.7 \mathrm{ml} / \mathrm{min} \\ \text { Injection vol..: } & 10 \mathrm{ul} \\ \text { Wavelength: } & 254 \mathrm{~nm} \\ \text { Sample Conc.: } & 1 \mathrm{mg} / \mathrm{ml}\end{array}$

Sample Conc:: $1 \mathrm{mg} / \mathrm{ml}$

Data File: $\quad$ D:IBIJUMMNT $\quad$ TNNY PROLINE ETHYL C5.dat

Method: $\quad$ C:EZChrom ElitelEnterprise $\quad$ Projects Default Method $c v r 12$.met

Acquired: 9/18/2016 4:22:38 PM $\quad$ Printed: 9/18/2016 4:54:44 PM
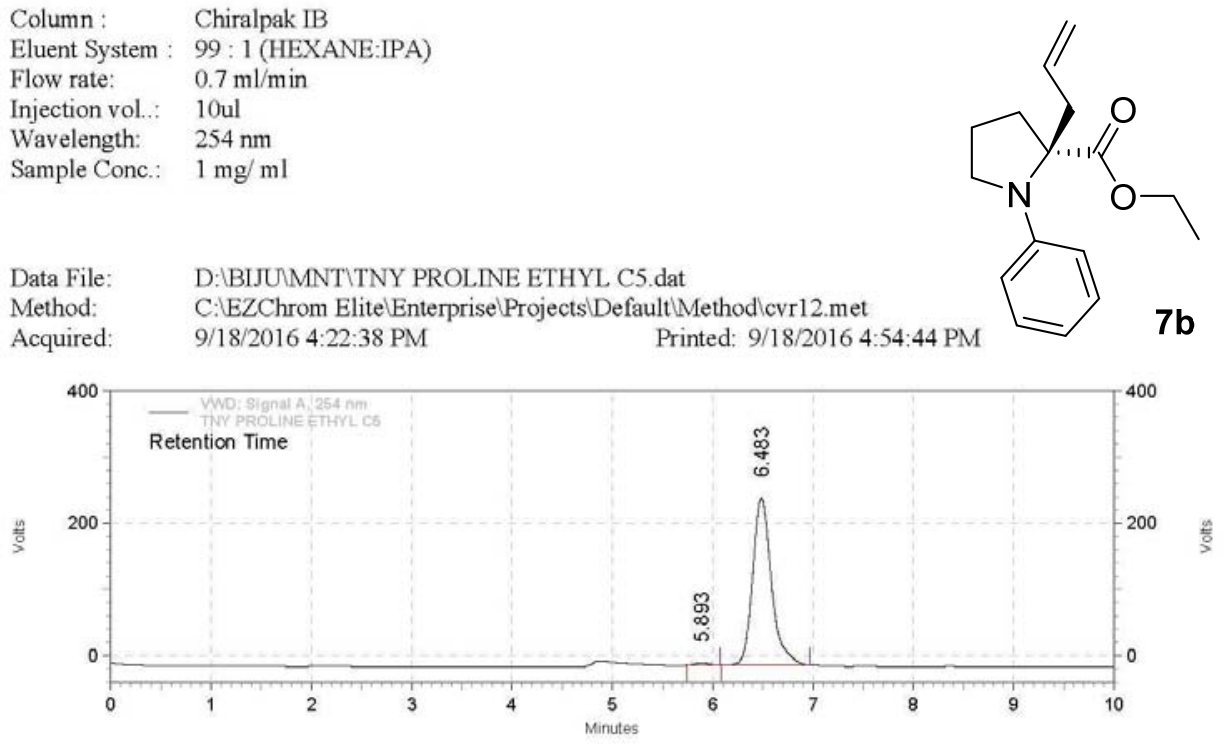

VWD: Signal A, 254 nm Results

\begin{tabular}{rrrr|}
\multicolumn{2}{r}{ Retention Time } & Area & Area \% \\
\hline 5.893 & 609488 & 1.09 \\
9.483 & 55061803 & 98.91 \\
\hline \multicolumn{2}{|r|}{ Totals } & & \\
& & 55671291 & 100.00 \\
\hline
\end{tabular}




\section{Methyl (S)-2-allyl-1-(benzo[d][1,3] dioxol-5-yl)pyrrolidine-2-carboxylate (7c)}

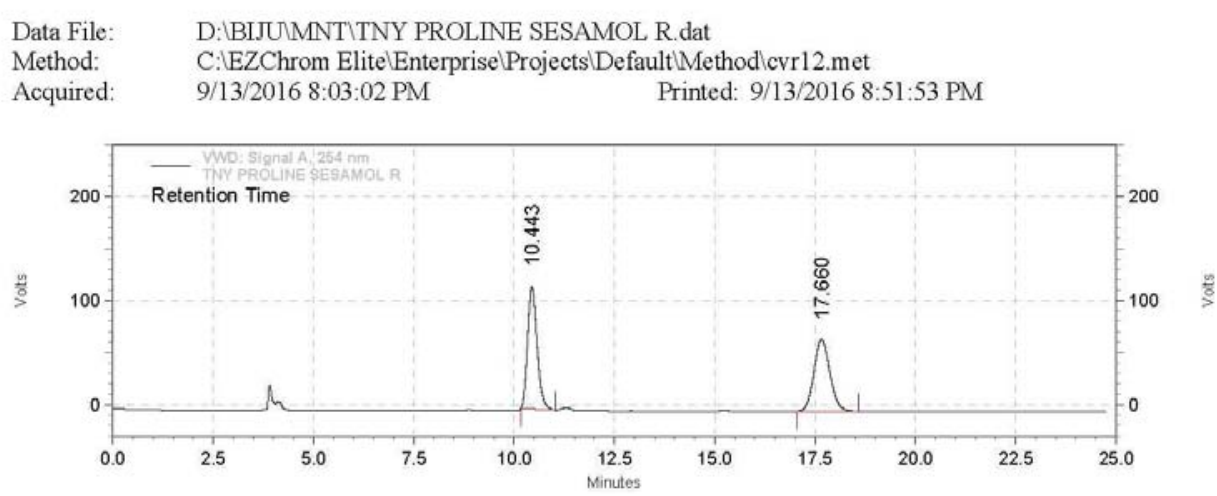

VWD: Signal A, $254 \mathrm{~nm}$ Results

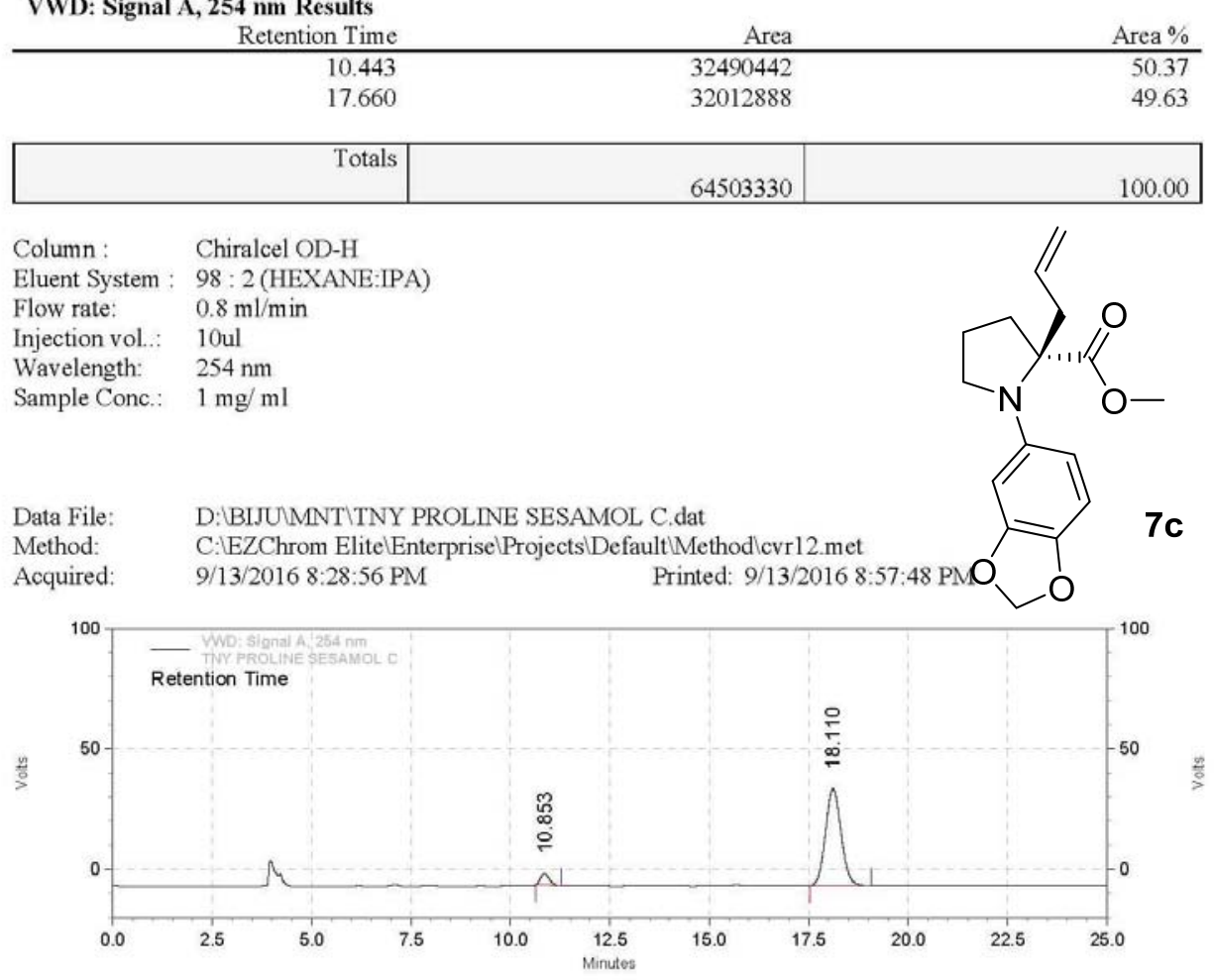

VWD: Signal A, 254 nm Results

Retention Time

10.853

Area

$1183709 \quad 6.04$

18.110

18398906

93.96

\begin{tabular}{|r|r|r|}
\hline Totals & 19582615 & 100.00 \\
\hline
\end{tabular}

